\title{
Sensor Fusion and MOE Development for Off-Line Traffic Analysis of Real Time Data
}
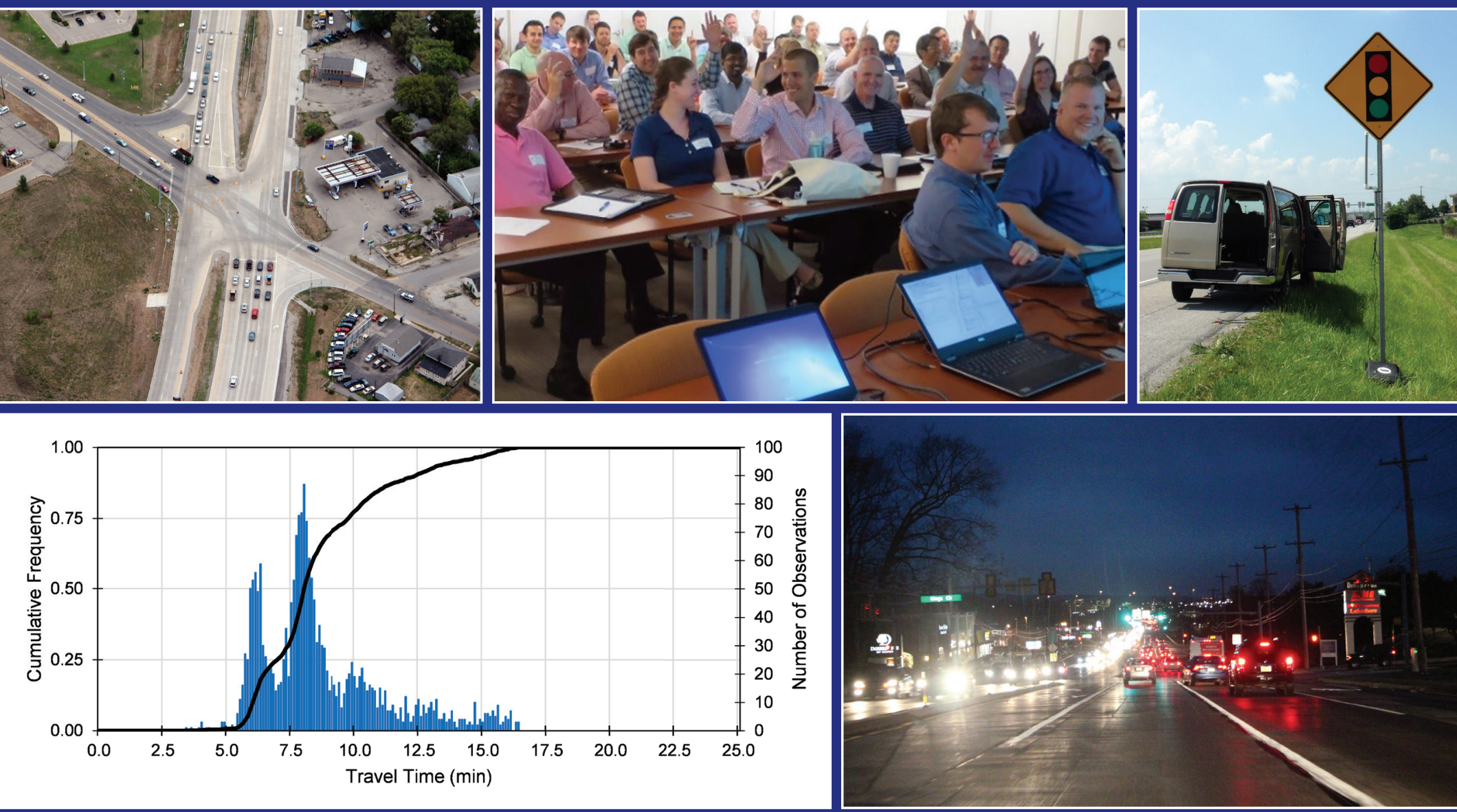

Christopher M. Day, Stanley E. Young, Darcy M. Bullock, Dennis So Ting Fong 


\title{
Sensor Fusion and MOE Development for Off-Line Traffic Analysis of Real Time Data
}

\author{
Christopher M. Day \\ Purdue University \\ Stanley E. Young \\ Traffax, Inc. \\ Darcy M. Bullock \\ Purdue University \\ Dennis So Ting Fong \\ Traffax, Inc.
}
SBIR Phase 3 Joint Transportation Research Project
Traffax, Inc.
Purdue University

August 9, 2017

\begin{tabular}{|l|l|}
\hline Deliverable Reference: & D3.2 Final Report \\
\hline Project Name: & $\begin{array}{l}\text { Sensor Fusion and MOE Development for Off-Line } \\
\text { Traffic Analysis of Real Time Data }\end{array}$ \\
\hline Contractor: & Traffax, Inc. \\
\hline Contract Number: & DTFH61-14-C-00035 \\
\hline Contract Term Start & $9 / 4 / 2014$ \\
\hline Contract Term End & $9 / 4 / 2017$ \\
\hline Key Personnel & Stan Young, Darcy Bullock, Dennis So Ting Fong \\
\hline
\end{tabular}




\section{Recommended Citation}

Day, C. M., S. E. Young, D. M. Bullock, and D. S. T. Fong. Sensor Fusion and MOE Development for Off-Line Traffic Analysis of Real Time Data. Purdue University, West Lafayette, Indiana, 2017. https://oi. org/10.5703/1288284316556

\section{Acknowledgments}

This work was supported by Traffax/USDOT SBIR DTFH6114C00035. The contents of this paper reflect the views of the authors, who are responsible for the facts and the accuracy of the data presented herein, and do not necessarily reflect the official views or policies of the sponsoring organizations. These contents do not constitute a standard, specification, or regulation. 


\section{Table of Contents}

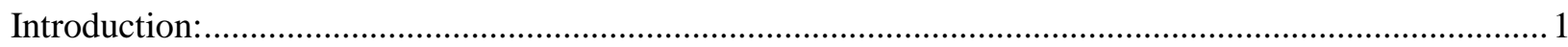

Chapter 1: New Data Sources and Emerging Arterial Management Concepts....................................... 3

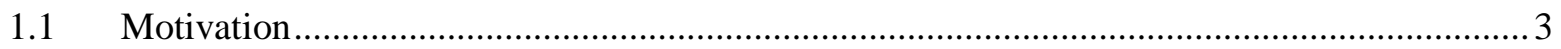

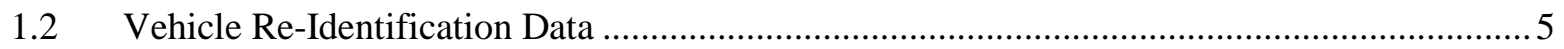

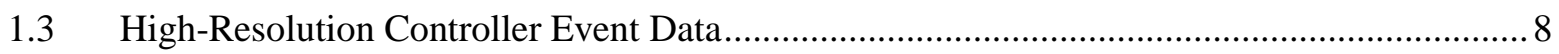

1.4 Synthesis: A New, Structured Approach for Analyzing Arterial Performance ...................... 11

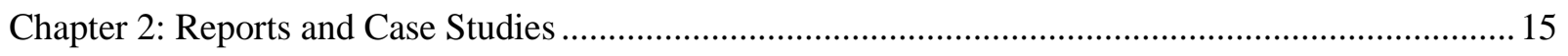

2.1 Performance Measures of Interrupted-Flow Roadways ...................................................... 15

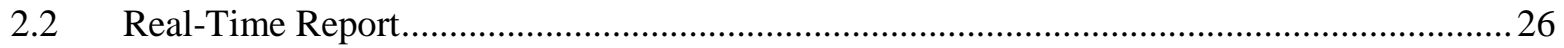

2.3 Arterial Trip Length Characteristics Report ....................................................................... 30

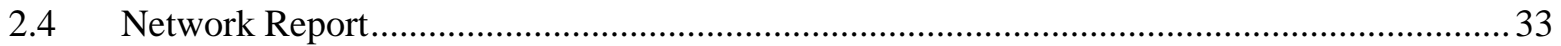

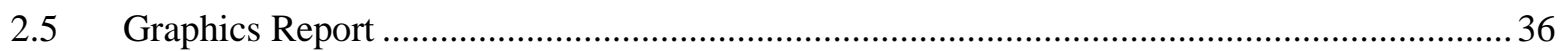

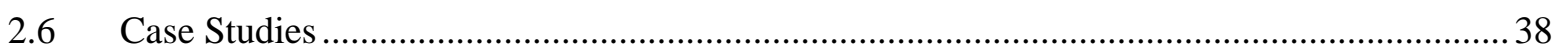

Chapter 3: Resources to Support Performance Measurement............................................................ 49

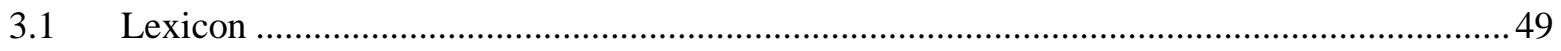

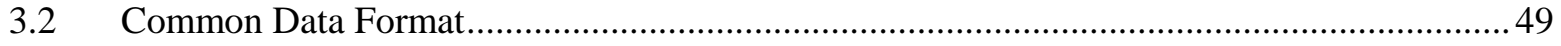

3.3 Arterial Performance Measures Software ............................................................................. 51

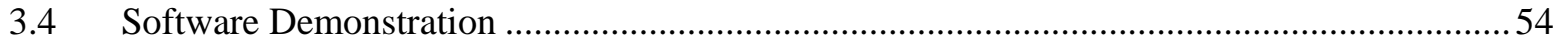

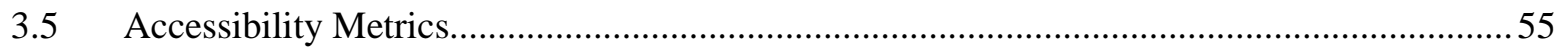

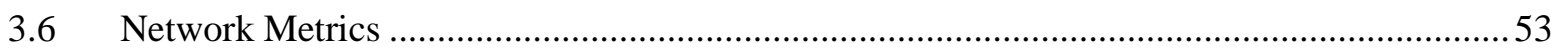



4.1 Georgia Department of Transportation (Atlanta, GA) ...................................................... 59

4.2 International Performance Measurement and Data Conference (Denver, CO)......................64

4.3 ITE Western District Annual Meeting (Las Vegas, NV) .....................................................6 66

$4.4 \quad$ NRITS Conference (Snowbird, UT) \& FLTDS (Orlando, FL) .............................................. 69

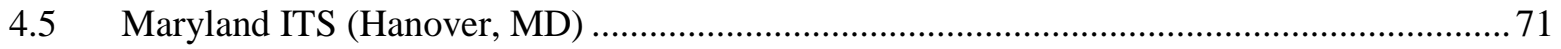



$5.1 \quad$ Vehicle Position Information in Emerging Data Sets ........................................................ 76

5.2 High-Resolution Data and Automated Traffic Signal Performance Measures ....................... 77

$5.3 \quad$ New Methods of Arterial System Management ................................................................. 79

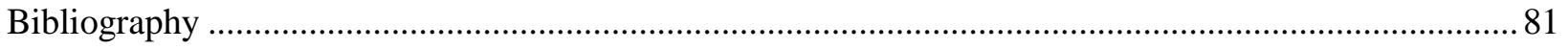




\section{Introduction:}

This final report presents the outcomes from the SBIR project Sensor Fusion and MOE Development for Off-Line Traffic Analysis of Real Time Data that was carried out in 2015 and 2016. This study explored the potentials of two new data sources, vehicle re-identification data and high-resolution controller event data, to improve the state of the practice in arterial traffic management. Chapter 1 of this report provides a brief introduction to the two new data sets that are envisioned as the enabling technologies for enhanced arterial management, and includes an overview of how they can be used in tandem for that purpose. The rest of the deliverables for this project advance those ideas further.

This project provided three sets of deliverables. The first group of deliverables was a collection of five reports and two case study whitepapers that explain the new technologies in depth, touch upon different aspects of their application, and include demonstrative examples of their use. These include:

- A four-module informational report drafted to introduce the new technologies in depth. It discusses their application to arterial management with regard to the two main aspects of signal system operation: corridor progression and capacity allocation. A shorter, Executive Summary style report was also written to provide an overview of this material.

- The potential uses of both data sets for real-time monitoring which are investigated in a second report.

- A third report which investigates arterial trip length characteristics through a field study using vehicle re-identification data, culminating with a distribution of measured trip lengths.

- A fourth report proposing a framework for arterial management at a network level, drawing parallels with approaches for assessing pavement condition.

- A fifth report focused on the use of graphical performance measures based on vehicle reidentification data and high-resolution data.

- Finally, two case studies that are carried out using two data sets to evaluate arterial performance in two different scenarios. The first looks at the use of high-resolution data to optimize offsets in a signalized corridor over a 5-year period. The data is used to identify a user benefit of approximately \$3.4 million obtained over that time. The second case study looks at conditions on an arterial highway where traffic from a parallel freeway was detoured onto the arterial. The length duration of the detour necessitated the development of a special signal timing plan, the impacts of which are examined with high-resolution data. The origin-destination characteristics of detour vehicles are also investigated.

This project final report includes a summary of each of these items in Chapter 2.

The second set of deliverables for this project encompasses tools to assist in implementation of methods and performance measures for arterial traffic management. This includes documentation of algorithms and associated software, standard data formats, and a lexicon of terms as described below:

- A lexicon for acronyms and terms is provided to clearly communicate definitions, concepts, and parameters for the implementation of software. 
- A common data format report for re-identification and high-resolution data was prepared that emphasizes data standards so that any resulting software from differing sources may be used independent of type or brand of re-identification technology and high-resolution controller data.

- Algorithms and software for arterial performance measures reliant on re-identification data were developed in an open source format for use as reference implementations. Software includes the implementation of a re-identification data standard (CWS5200), the analytics processing, and the visualization routines.

- The arterial performance measures open source software was extended to support network level travel time and reliability metrics identified to support a network level arterial management framework.

- A recorded demonstration was prepared explaining the purpose, development, framework, and potential uses of the developed open-source software.

- Existing analysis software (BluSTATs) was extended to capture the roadway trip length characteristic (RTLC) metric defined in initial research efforts.

Chapter 3 provides a summary of each these items.

The third set of deliverables for this project was the presentation of the material in a series of public forums, allowing for dialog with practitioners, giving them a chance to provide feedback and comment on the direction of the methodology and its potential. Six public forums were conducted in 2015 as part of this research in six different locations across the US. Chapter 4 presents an overview of those forums and summarizes the feedback obtained from the attendees.

Finally, Chapter 5 concludes this report with a discussion of the future directions of the methodology, taking into consideration recent trends in signal data, and connecting the present research to some related research in the area of performance-based arterial management. 


\section{Chapter 1: New Data Sources and Emerging Arterial Management Concepts}

\subsection{Motivation}

In the $21^{\text {st }}$ century, transportation agencies have seen their pools of available resources decline as their revenues have not increased proportionately to the costs of maintaining the infrastructure. In the past, construction of new facilities carried a highly visible benefit that would allow for systems to be improved by adding new capacity. As the overall shape of transportation networks have matured, the need for maintenance of the existing system has grown in importance. The current state of affairs demands that agencies extract the best performance of their system by the most efficient means, and that those agencies demonstrate accountability in the stewardship of the resources given by the public. This has been underscored by the emphasis on performance measurement in recent highway funding bills.

Arterial traffic systems have rather complex needs in that their performance is dependent not only on the capacity provided in their geometric design, but also on the management of that capacity by traffic control devices. Traffic signals are the principal means of controlling traffic on major arterial roadways with atgrade intersections. Although traffic signal controllers are in essence hardened computers, advanced data collection capabilities have historically been difficult to realize, for a number of reasons. Until recently, most controllers did not possess enough memory or computing power to support detailed data collection. Even if they did, it was expensive to establish good communication systems with a large inventory of intersections that would in some cases be distributed over a wide geographic area. Advanced systems that did provide these capabilities were quite expensive, entirely proprietary, and not interoperable with each other. This limited their use to selected areas with the greatest need. Furthermore, there was no common concept of what kinds of data a system might collect, other than the occupancy and volume metrics used in traffic responsive control.

As a consequence, traffic signal operations have often fared poorly, particularly in areas where the managing agencies have lacked the resources to invest in proactive maintenance programs. The National Traffic Signal Report Card [1], an industry self-assessment carried out several times between 2005 and 2012, repeatedly yielded low overall scores for the US, and consistently produced a subscore of " $F$ " for the category of traffic monitoring and data collection. In short, very few agencies were collecting data on a widespread basis at a scale necessary to keep up with changes in traffic levels. Agencies relied on manual methods, such as manual turning movement counts and floating-car studies, to understand how their systems were doing. Due to the expense of the data collection, such studies would typically only be undertaken when signalized corridors were being retimed and the schedule for carrying out retiming activities was usually arbitrary. That is, the attention spent on maintaining systems was often disproportionate to the need.

The state of arterial management stands in great contrast to that of pavement management. The domain of pavement management is today quite mature. There are standard metrics for measuring the quality of pavement and well-established means of using that information to program resources to maintain an inventory of roadways. Figure 1.1 contrasts the history of this development with that of arterial management systems. As can be seen here, efforts to quantify pavement condition began in the late 1950s 
[2], and had become commonplace in engineering practice by the 1990s. Today, decisions on repaving and reconstruction of roadways can be informed by nearly complete knowledge of the pavement condition across an agency's inventory, using a common metric, such as the International Roughness Index (IRI). New construction can be evaluated with such data, and it is even possible to implement performance-based contracting on such a basis.

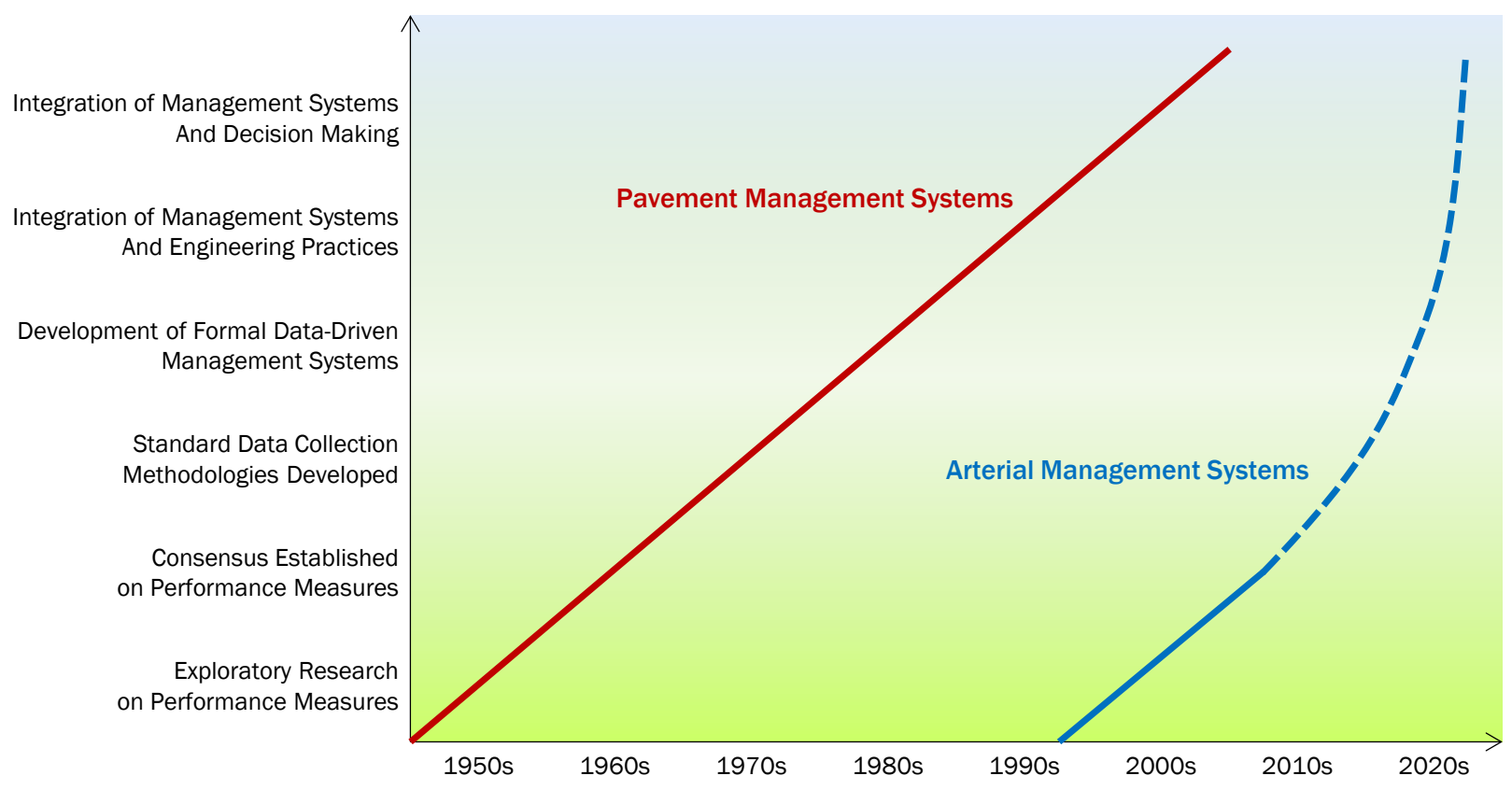

Figure 1.1 Evolution of pavement management systems and arterial management systems

Arterial management systems have lagged behind. This is somewhat due to the complexity of traffic management. Whereas pavement quality tends to degrade predictably with the exposure of pavement to weather and traffic volumes, traffic demand is less predictable and experiences considerable variation spatially and temporarily.

In the past, traffic conditions were usually measured by manual methods during specific traffic studies. These included turning movement counts, which could be used in a modeling system such as the Highway Capacity Manual [3] to determine metrics like the Level of Service; and floating-car travel times. In lieu of having such metrics on a widespread basis, often times agencies would instead measure the amount of effort expended. Examples of such metrics would include the number of signal retiming activities carried out, or the amount of time between consecutive retiming activities.

Extensive instrumentation is needed to automatically measure changes on a network scale. The basic equipment needed for automated measurements-detectors-have been in place for many years, but scalable data infrastructure to record, transmit, and process the data has only emerged recently. Scalability relies on the components being inexpensive enough to distribute throughout the network. In the past 10-15 years, the following technologies have arisen. 
- One is the development of high-resolution data, which records the measureable events at a signalized intersection. The controller (or an inexpensive cabinet widget) monitors the state of each detector and signal head output, among other things, and records the times when any of these states change. This can be used to develop a number of performance measures that quantify the performance of different aspects of the signal operation, in microscopic detail. This type of data can make use of the existing components of a conventional traffic control system.

- Another is the development of vehicle re-identification data. That is, some unique identifier associated with a sample of individual vehicles can be identified relative to the location and time that it is identified. This can then be used to understand the movement of traffic. Travel times between multiple locations can be measured by re-identifying vehicles. An example would be matching of Bluetooth MAC addresses by onboard mobile devices at multiple locations. This type of data represents a new functionality for a conventional traffic control system, but the equipment needed for re-identification is relatively inexpensive.

With the advent of these technologies, a number of possibilities for arterial management have emerged. Several studies on automated performance measures based on high-resolution data have extensively demonstrated the breadth of their application to all aspects of signal operation at traffic signals—such as allocation of capacity [4] [5], quality of progression [6] [7] [8], safety of users [9], and equipment maintenance [10]. Vehicle re-identification methods have also been used in arterial management, providing a way to directly measure the impact of that operation on user travel times [11] [12] and origindestination characteristics [13]. Because the two datasets essentially work in separate domains, they are most powerful when combined together [6]. User impacts can be quantified by vehicle re-identification, while the reasons for those impacts can be deduced by performance measures based on high-resolution data. Rather than relying solely on intuitive or anecdotal knowledge of the system and making inferences on the level of investment, agencies can now adopt a data-driven process, more similar to that already used in pavement management systems.

\subsection{Vehicle Re-Identification Data}

One of the key performance indicators for the quality of service given by an arterial highway to its users is the travel time needed to make it from one end of a highway section to another. Any increase in that travel time beyond the amount needed for travel at the free flow speed represents the delay incurred due to slowing and stopping caused by congestion and traffic control. In the past, travel times could be measured by floating-car studies, or by license plate matching. In the floating car study, the analyst would collect data by driving a probe vehicle up and down the corridor and measuring the travel time directly in that manner. In license plate matching, observers would record license plate numbers at two different locations in the system and note the time of observation. By matching the plate numbers and comparing the times, they could infer the time needed for the vehicle to travel from one location to the other.

Automated vehicle re-identification works in much the same way as license plate matching. In this case there needs to be some method of identification that is easy to obtain in an automated fashion. Bluetooth MAC address matching emerged about 10 years ago as an easy and scalable means of doing so, and is still in rather widespread use at the time of writing. MAC addresses are quasi-unique identifiers that are often broadcast by mobile devices when using the Bluetooth protocol to wirelessly connect devices, such as a headset to a cell phone. Any other device within range can read such a broadcast and observe the MAC address. Thus, by putting a Bluetooth transceiver within range of a roadway, an observer can 
develop a list of timestamped observations of unique vehicle IDs. When multiple transceivers are used at multiple locations, their lists are compared to deduce the travel time.

Figure 1.2 illustrates the concept of vehicle re-identification. In this case, a vehicle with particularly unique characteristics is observed at two different places in a traffic network-in this case, during a special event (a football game at a university stadium). The vehicle was first observed at a stadium, and then observed again elsewhere, on a local arterial while most likely en route to an Interstate highway. From the time of the two photographs, it is a simple matter to compute the travel time.

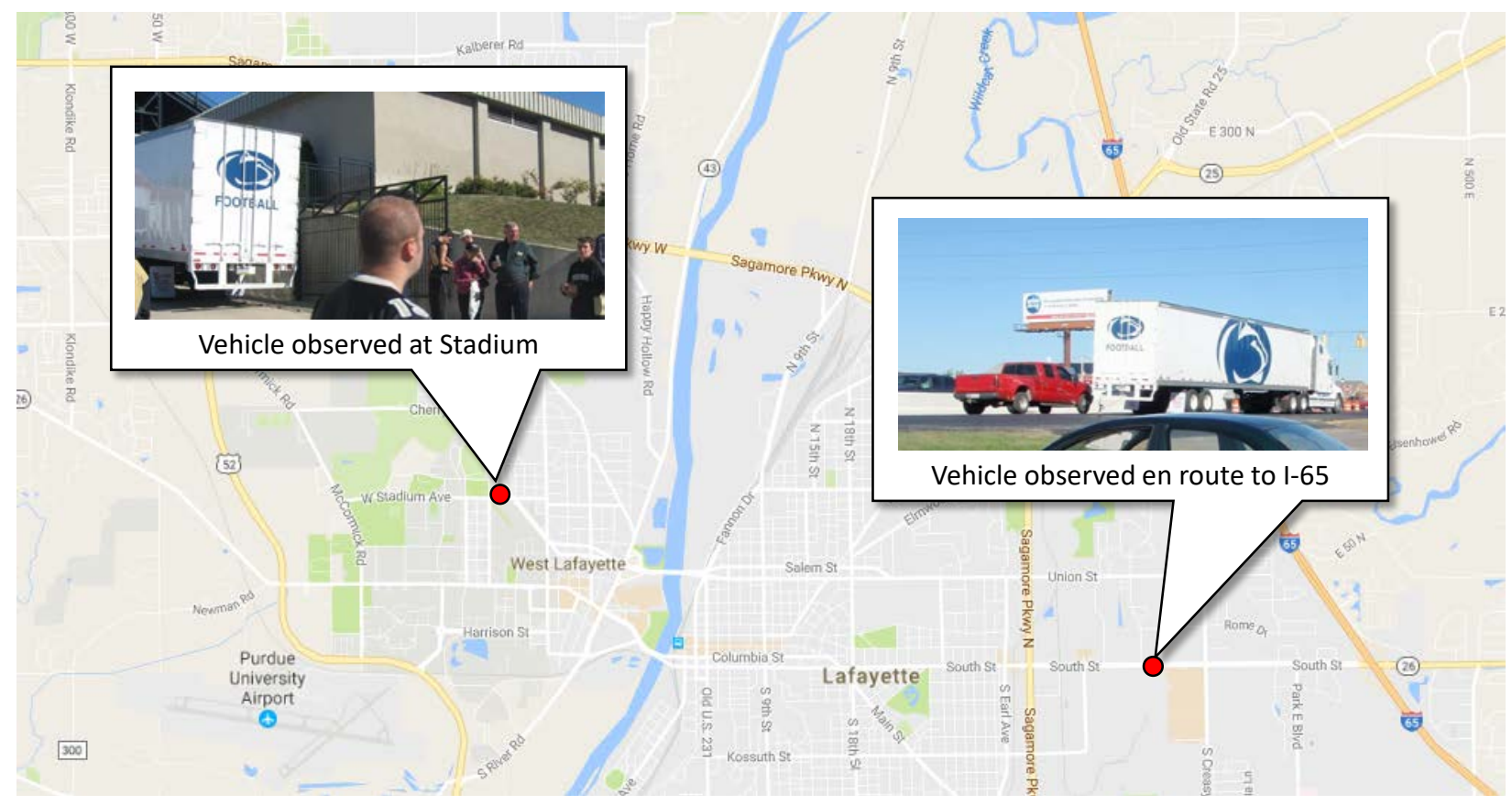

Figure 1.2 Vehicle re-identification data concept

The potential of this type of information becomes much clearer when many individual data points are brought together. Figure 1.3 shows a scatterplot of individual travel time observations from one direction along an arterial highway, including data from all of the Wednesdays within a 6-month period. The vertical red lines indicate times of day when the signal timing plan changes from one pattern to another. The point cloud shows how travel times change through the day. The impact of the signal timing is illustrated by how the patterns in the travel times change. There are a number of other things that can be observed. For example, in the PM period (15:00-19:00), the range of typical travel times is between 6 and 10 minutes, but there are three horizontal "bands" in the distribution showing a tri-modal distribution, with the different modes explained by places where vehicles were stopped at traffic signals. 




Figure 1.3 Example of vehicle re-identification data in a signalized corridor

The data can be still more valuable when extracted to summary metrics to facilitate comparisons; changes in travel time before and after an investment are particularly useful. A number of aggregate metrics can be used to reduce a travel time distribution to a single number. A compromise between the detail of the raw data and the simplicity of a single value is the cumulative frequency of the distribution, which can be displayed as a single curved line called a cumulative frequency diagram (CFD), also called a cumulative distribution function (CDF). Figure 1.4 shows an example of CFDs for two directions along a roadway for two conditions, before and after an update to the signal timing plan. Each line shows the shape of the distribution in a cumulative form, the vertical axis representing different percentiles of the distribution and the horizontal showing what travel times are observed at that value. The "After" line is entirely to the left of the "Before" line, showing reductions in travel time; the arrows show the magnitudes of those reductions at the median and the $25^{\text {th }}$ and $75^{\text {th }}$ percentiles. In this case, the travel times are reduced by about a minute, or what appears to be roughly $20 \%$ of the original travel time, in both directions.
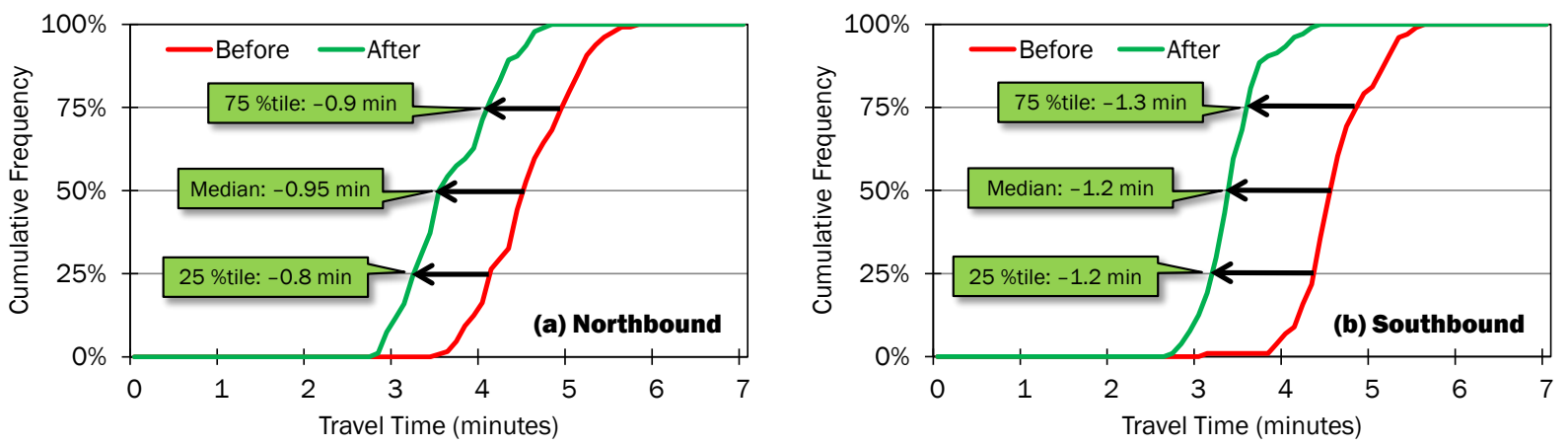

Figure 1.4 Comparison of changes in travel time using cumulative frequency diagrams

The reports produced in this project extensively explore the uses of vehicle re-identification data for understanding current travel times in an arterial network, knowing how they are trending, and how they change under interventions. This can provide a very powerful tool for managing an arterial system, as the performance of individual corridors can be assessed and ranked to understand where to invest resources 
for retiming. However, this data itself does not shed light on why travel time increased or decreased. Although in Figure 1.4 it is clear that travel times have been reduced, the reason why this reduction occurred is not known from the data by itself. It is possible that traffic patterns were changed by the amended signal timing, but it is also possible that there was congestion in the "before" case that increased the travel times that was absent in the "after" case. High-resolution data can be used to develop some of those insights.

\subsection{High-Resolution Controller Event Data}

Signal timing plans are typically designed with the assistance of software tools, based on intersection and corridor geometry along with expected traffic volumes. After signal timing is deployed in the field, engineers often observe traffic in order to ascertain whether adjustments are needed, since no software model can completely predict field conditions. This process greatly improves the quality of the timing, but it is sharply limited by to the amount of time that the analyst can spend in the field making observations and responding to them. Some agencies have access to video cameras that enable operations to be observed remotely, and this helps them multiply their efforts. However, even then, the capability of the operators to make adjustments are still limited to the periods when they can spend time to make the observations.

High-resolution data offers a way for these types of observations to be made automatically, by means of analysis of the events at intersections and by making some inferences using observations from the events. An "event" is a state change, of which the two main states of interest are those of the signal head outputs (red, yellow, or green for vehicles, and walk, flashing don't walk, and don't walk for pedestrians) and the detector inputs (the zone is occupied or unoccupied). Although this is less information than a complete visual representation of the corridor, when an intersection has enough detection to cover enough of its movements, it can provide enough information to identify where further attention should be paid to the operation. Thus, in lieu of having a human observer present at all times at the intersection, the data can be present at all times and provide a good estimate of the quality of operation.

One major operational consideration at a traffic signal, and one that significantly affects arterial travel times, is the quality of signal coordination: that is, whether traffic patterns facilitate the smooth flow of vehicles along the corridor and through multiple intersections. Figure 1.5 shows a time-space diagram showing vehicle trajectories as lines moving diagonally across the plot. The red horizontal lines show the location of the stop bar and the times when the signal is red. The trajectories do not cross the line, instead becoming horizontal here: these are stopped vehicles. The blue horizontal line shows the location of a detector situated $400 \mathrm{ft}$ upstream of the stop bar. The black dots where the trajectories intersect the detector line show the times of detection. For the signal controller, this would be where the detector turns ON when a vehicle passes over it. This is the only information that the controller knows - the rest of the trajectory is invisible to it. However, when combined with the timing data, it provides a powerful piece of information. The dashed red and green lines project the signal timing upward to a diagram above the plot, which shows the red and green intervals in one cycle. On top of this, the detection times are also projected, with some in red and others in green-in this case it is clear that the majority of vehicles arrive in green, which is preferable. 


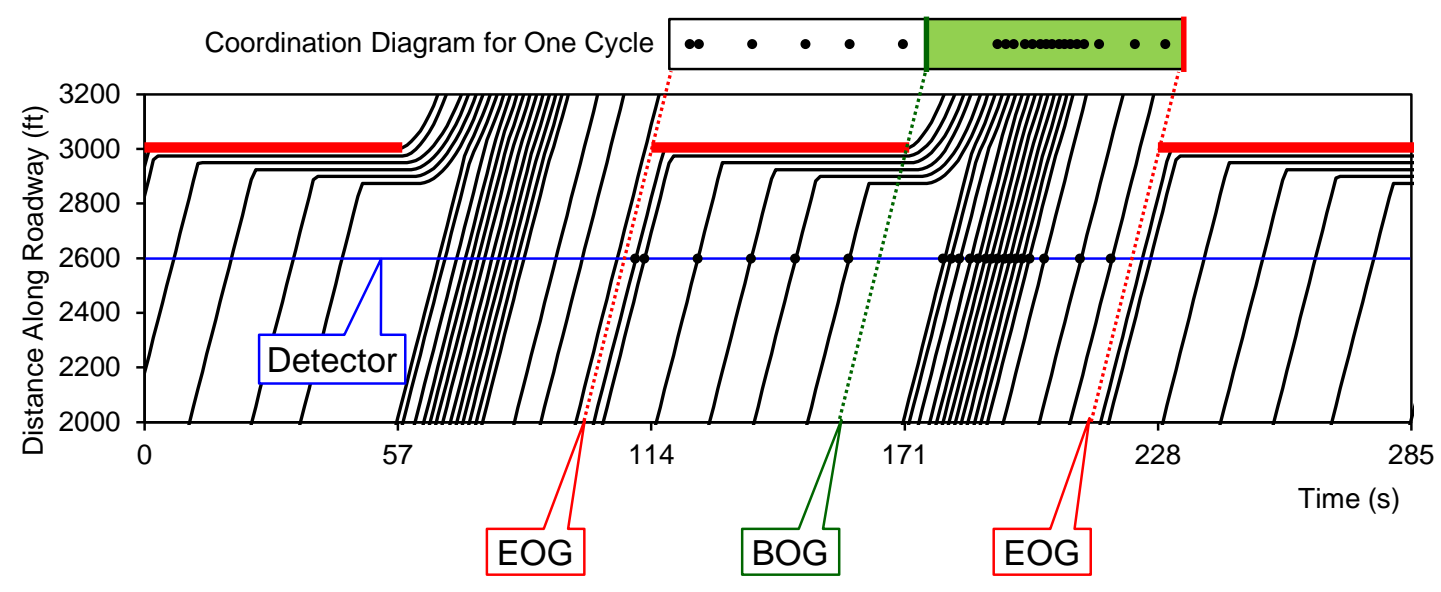

Figure 1.5 High-resolution controller event data concept

In the same way that the travel time data becomes more powerful when data from multiple vehicles are combined, this particular view of the intersection state becomes more powerful when combined with that of multiple cycles. Figure 1.6 shows this view. Here, the diagram at the top of Figure 1.5 has been produced for all of the signal cycles over a 24-hour period; it is shown vertically rather than horizontally, with the shaded green region again representing the time in every cycle where the signal was green, the white region showing when it was red, and the dots showing the time of vehicle arrivals relative to the state. The green and red lines show the time of the beginning of green (BOG) and end of green (EOG) respectively. In this example, over most of the day, the largest distribution of the arrivals are coincident with green, meaning that most vehicles are progressed rather than stopped. This chart, called a "Purdue Coordination Diagram” (PCD), illustrates conditions for one approach at a signalized intersection.

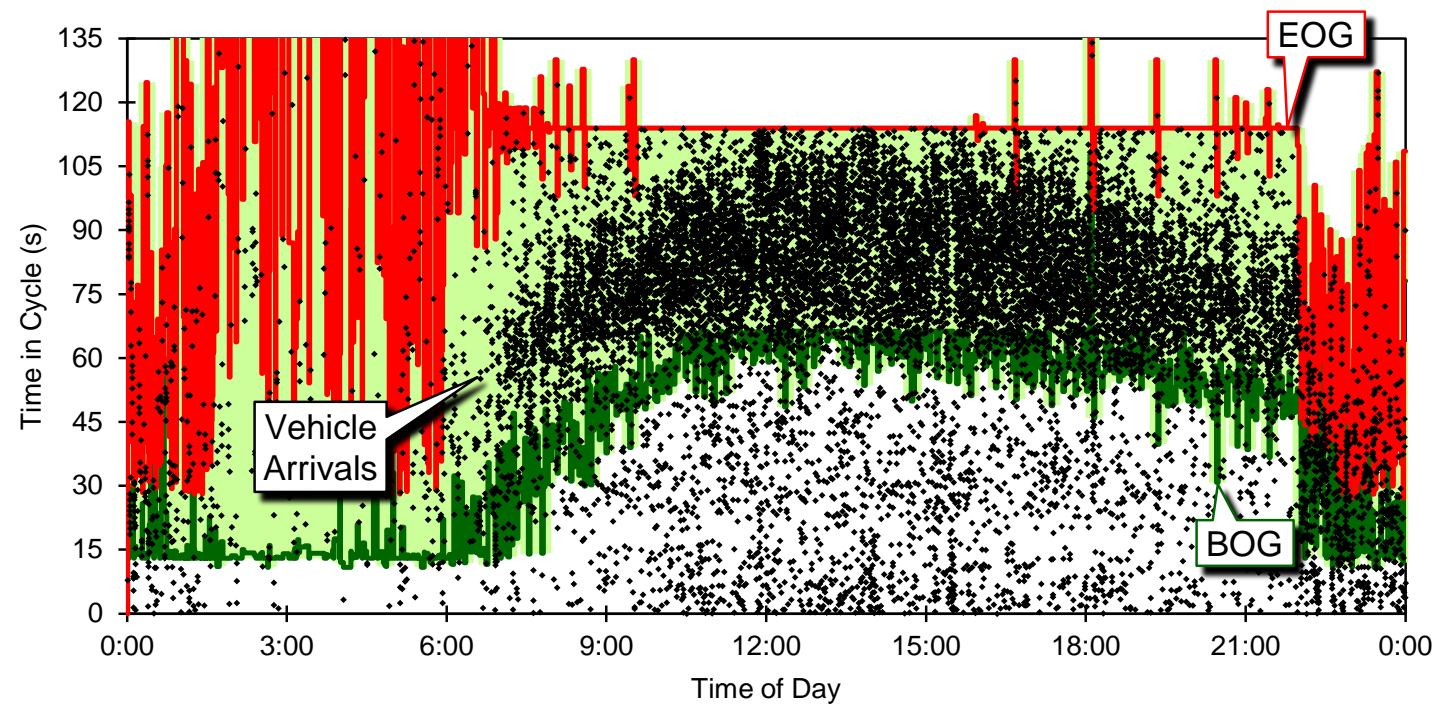

Figure 1.6 Example of a graphical performance measure: the Purdue Coordination Diagram (PCD) 
The microscopic detail provided by the PCD can be summarized in a number of various metrics; the basic information here consists of the number of arrivals in red and in green, and the durations of green. One simple metric that succinctly conveys the quality of progression is the percent on green (POG). Figure 1.7 shows a collection of charts for a corridor that explains how POG changed before and after an update of the signal timing. Each pie chart shows the condition for an individual approach; there are two approaches along the mainline at each intersection. The dark slices of the pies show where POG increased, the red slices where it decreased.

Most of the approaches in the southern four intersections exhibit large dark-green slices, showing that POG increased greatly in that area. This data corresponds directly to the travel time changes illustrated in Figure 1.4, helping correlate the signal operation with the impact on the facility users. This summary view is built on the microscopic data, which is still available to the analyst who wishes to "drill down" to the next level and examine the performance of an individual approach in detail.

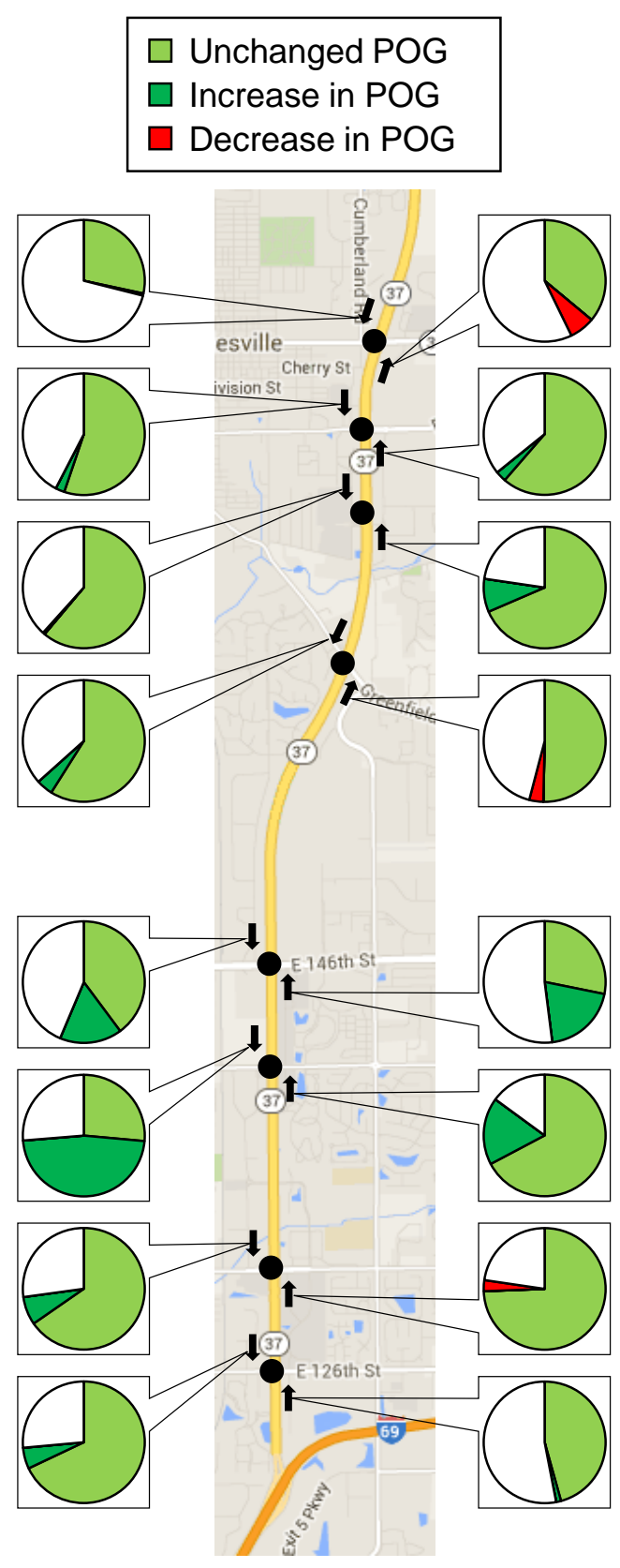

Figure 1.7 Example before/after assessment of percent on green using high-resolution data 


\subsection{Synthesis: A New, Structured Approach for Analyzing Arterial Performance}

Vehicle re-identification data and high-resolution data provide intelligence on arterial operations and, when combined together, enable a powerful set of tools to be developed. However, the complexity of arterial operation, particularly its temporal and spatial variation, demands that a structured approach be taken to make use of such tools to the greatest advantage. Already in the examples presented here, the notion of there being aspects of operation relevant to individual movements, to intersections, and to corridors has been shown, and it has been seen that performance will differ with the time series selected for analysis: time of day, day of week, and so forth.

This research proposes a structured approach to the analysis that uses different levels that go from holistic, system-level views, down to individual signalized movements. Figure 1.8 shows a graphical example of how this might break down for an agency.

- At the network or " $50,000 \mathrm{ft}$ " level, the entire arterial program is of interest. Agencies make planning and programming decisions at such a level, and resources for operations and maintenance are distributed according to need. The overall plan for running the system is relevant; interaction with other systems as well as other agencies is also important. The performance measures at this level consist of aggregations of the lower levels and quality of service provided by the system. For example, the travel time between selected origins and destinations would make a good high-level performance measure.

- At the corridor or "5000 ft" level, the scope is narrowed to an individual roadway facility, which in an arterial would typically be a corridor or a localized grid area comprising several intersections. The performance measures may consist of direct measurements of travel time at the corridor level as well as aggregations of intersection-level metrics. At this point, relevant performance measures would include the travel time characteristics of the corridor and the existence of deficiencies in capacity or anomalies in the operation. Sometimes multiple corridors work together in a system, perhaps varying with time of day, and perhaps affecting operation of freeways and other facilities.

- At the intersection or "500 ft" level, the analyst becomes more concerned with the details of individual movements. Here, the performance measures of specific movements become important. When zoomed in at this level, it is likely that a deficiency is already known from higher level analysis; the details can be helpful to assess ways to remedy the situation and to evaluate the effects of an action. 
Network Level (50,000 ft)

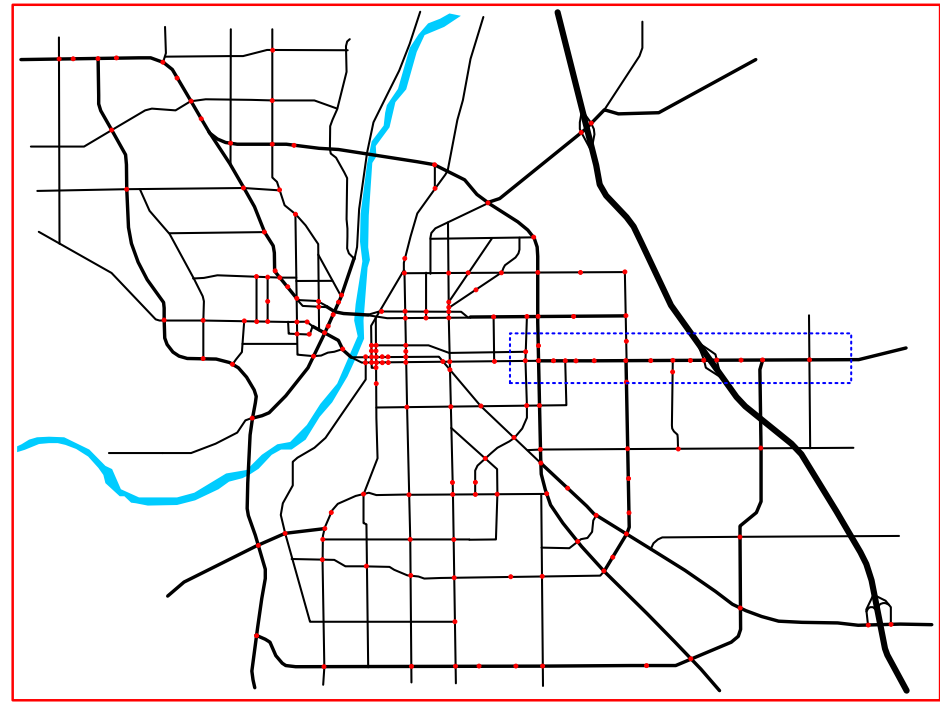

Corridor Level (5000 ft)

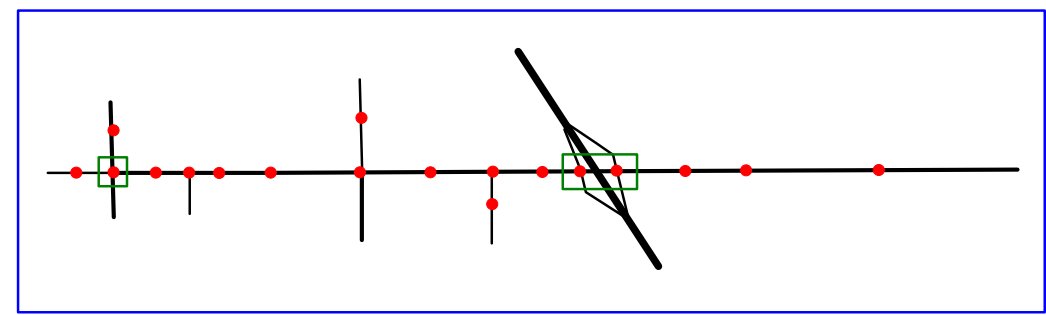

Intersection Level (500 ft)
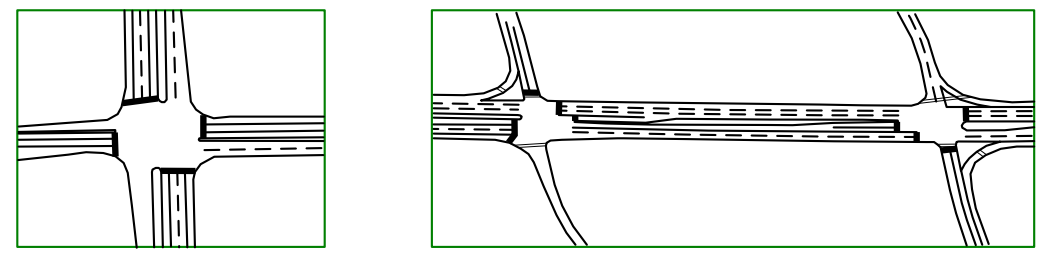

Figure 1.8 Analysis levels for arterial management 
Another way to structure the analysis of arterial operations is to divide the activities into groups. Figure 1.9 shows a chart that illustrates the idea. Under the overall umbrella of management, the activities of monitoring, analysis, and improvement are supported by performance measures. Arterial operation can be divided into two functions based on the spatial organization of systems. System control involves the coordination of multiple intersections on a corridor for progression, while local control is concerned with phase-switching at a single intersection. Different components can be seen to be relevant to each activity under operation.

These two perspectives on performance map into two categories of operational concerns that would be considered for system management. Progression is the function of a signal system whereby traffic is moved along multiple signals in a corridor, ideally with little to no stopping and with little delay when stops are incurred. This involves system control; it affects mostly the major movements, and the effectiveness can be measured with travel times as well as with progression metrics at the intersection level. Capacity allocation is the function of a signalized intersection where competing traffic demands of different movements are reconciled by the amounts of green time apportioned to them. This is the main function of local control, operating with the constraints needed to meet the demands of system control. All of the movements are affected, not only those serving the mainline along roadways. The effectiveness can be measured using intersection level metrics, and aggregated to higher levels.

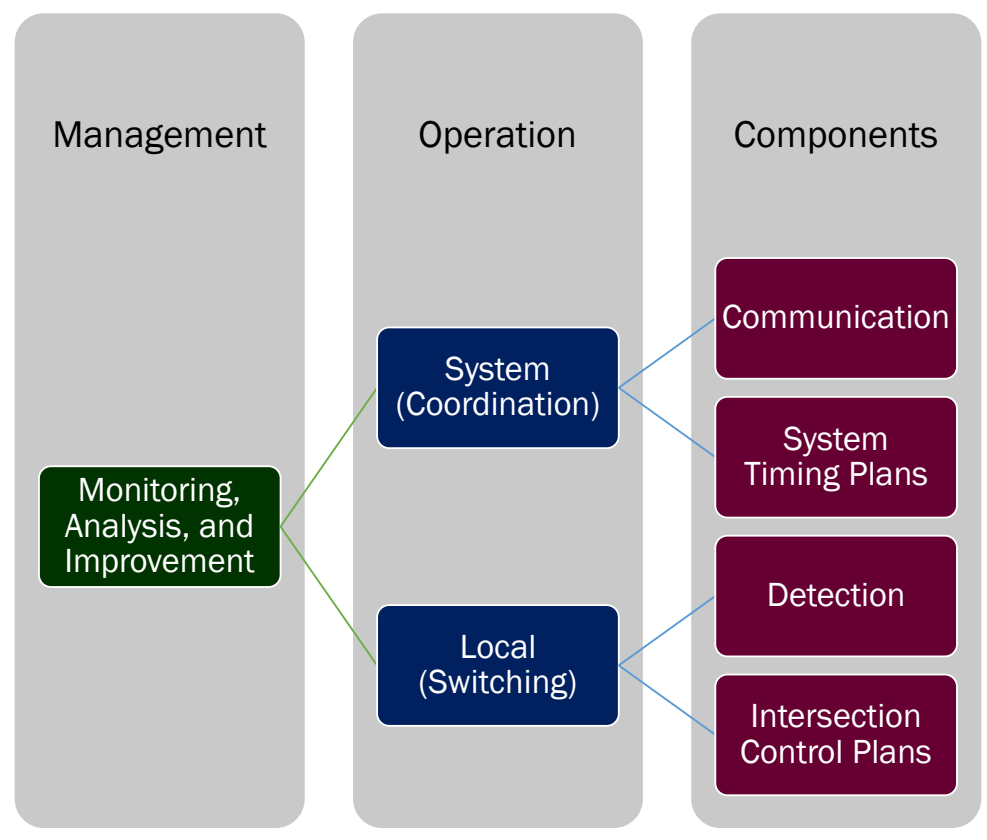

Figure 1.9 Breakdown of arterial management into components 
This research synthesizes these concepts into a unified vision for establishing condition metrics for managing arterial systems at a broad level, in the Network Report. Concepts from pavement management are reviewed to gain perspective on an effective way of assessing conditions for a large inventory of roadway facilities. In pavement management, ratings of different aspects of pavement conditions are used to develop a composite index for roadway sections. These can then be used to evaluate the overall network - for example by considering the percent of the system in good condition or in poor condition. Similarly, the same concepts can be applied to arterial management, by considering the aspects of evaluation and how to roll them up into condition indicators.

The Network Report takes on this problem and proposes a framework to develop it. Figure 1.10 illustrates the basic idea. The core elements of the analysis are the basic definitions of the roadway segments and their attributes. The next level that builds on those definitions is the condition ratings, which is where data is integrated and given context by comparison to thresholds. Finally, that condition data is aggregated and used to develop performance indicators at a network level. With such a framework, it becomes feasible to consider high level metrics that reveal the overall state of an arterial system, track changes over time, and after perhaps enough data has been collected to understand the potential impacts of different treatments, predict the changes in performance that can be expected with investments.



Figure 1.10 Network performance framework 


\section{Chapter 2: Reports and Case Studies}

This project generated one four-module report that covered performance measures in detail, four additional reports discussing topics related to applications of those performance measures, and two case studies that demonstrated the use of the performance measures to evaluate conditions in two different real-world operational scenarios. This material is one part of the deliverables of the project. This chapter summarizes the content of those reports.

\subsection{Performance Measures of Interrupted-Flow Roadways}

The report entitled "Performance Measures of Interrupted-Flow Roadways Using Re-Identification and Signal Controller Data" summarizes the use of performance measures generated from vehicle reidentification data and high-resolution event data from signal controllers to evaluate arterial roadways. The report relies on the use of conceptual "levels" on which an analysis takes place: intersection, corridor, and network. The material is organized into four modules that can be presented independently:

- Module 1 presents basic concepts for arterial management, including a discussion of objectives for arterial operation and measurable items at each level.

- Module 2 discusses vehicle re-identification data and high-resolution controller event data in detail, including basic data requirements and some example applications.

- Module 3 discusses performance measures oriented toward managing travel times through an arterial system. These are used to evaluate progression through traffic signals and the associated impact on route travel times through signalized intersections.

- Module 4 discusses performance measures oriented toward managing capacity allocation at signalized intersections. These are used to evaluate the utilization of capacity and locations where demand exceeds capacity in a network of arterials.

The four modules each include several examples drawn from field evaluation of traffic signal systems using vehicle re-identification and high-resolution controller event data. The report also includes a shorter executive summary that can be used to communicate the essential concepts of the full report to less technical audiences, including agency executive staff and the public.

\subsubsection{Module 1 Overview}

The key to a performance evaluation of any system, including an arterial network, is to determine the objectives of the system operation. The evaluation can then determine the degree to which the system meets those objectives. Different agencies will have different objectives depending on their own policies, and these will vary with the context of the arterials, such as their environment and geographic distribution. Table 2.1 shows an example of how two different stakeholders might have different perspectives on objective priorities for the same system. Stakeholder A is more interested in management of vehicle movements, while for Stakeholder B these are secondary to improving service for pedestrians and bicycles. These objectives are likely to evolve over time as well, especially as the characteristics of service change in response to changes driven by the objectives. The selection of performance measures should relate strongly to the desired objectives for the system.

The emergence of new data sets has made it possible to begin developing more detailed information about operations than has been possible in the past. This project has focused on two data sets in particular, vehicle re-identification data and high-resolution controller event data. Module 1 of the report presents 
some background on these data sets and discusses performance measures that can be developed from them at different levels (intersection, corridor, and network). Table 2.2 presents a mapping of potential performance measures from these data sets against different objectives that could be considered for evaluating an arterial system. Several examples are given for performance measures at the network, corridor, and intersection levels.

Another important topic is about understanding how to implement performance measures. The report briefly touches on some specific tasks and roles of agency personnel as they relate to performance measures. Table 2.3 shows a summary of typical personnel roles. Importantly, the implementation of a system to manage the data sets, which are required to support performance measures from vehicle reidentification and high-resolution data, introduces some tasks that may be new for some agencies. This includes the development of a system to support the storage and retrieval of the data, as well as some "data curation" tasks needed to maintain data in the long term, such as whether or not to archive the data beyond some horizon.

Table 2.1 Example objectives and how they might be prioritized between two stakeholders

\begin{tabular}{|l|r|r|}
\hline Objective & Stakeholder A & Stakeholder B \\
\hline Improve Progression & 1 & 4 \\
\hline Improve Capacity Allocation & 2 & 3 \\
\hline Improve Pedestrian Service & 3 & 1 \\
\hline Improve Bicycle Service & 4 & 2 \\
\hline Maintain Working Detection & 5 & 8 \\
\hline Maintain Working Preemption & 6 & 9 \\
\hline Minimize Pollution and Noise & 7 & 5 \\
\hline Automate Traffic Counts & 8 & 6 \\
\hline Develop Origin-Destination Data & 9 & 7 \\
\hline
\end{tabular}


Table 2.2 An example of matching potential performance measures to possible objectives

\begin{tabular}{|c|c|c|c|c|c|c|c|c|c|c|c|}
\hline \multirow[b]{2}{*}{ Objective } & \multicolumn{11}{|c|}{ Performance Measure } \\
\hline & 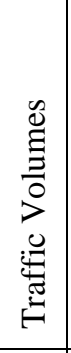 &  & $\begin{array}{l}\stackrel{.}{\pi} \\
\widetilde{\sim} \\
\bigcup \\
\supset\end{array}$ & 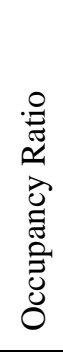 & 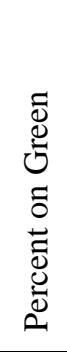 & 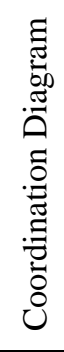 & 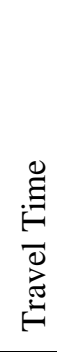 & 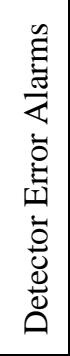 & 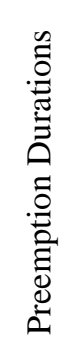 & 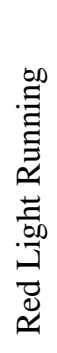 & 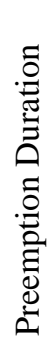 \\
\hline Improve Progression & & & & & $\mathrm{X}$ & $\mathrm{X}$ & $\mathrm{X}$ & & & & \\
\hline Improve Capacity Allocation & $\mathrm{X}$ & $\mathrm{X}$ & $\mathrm{X}$ & $\mathrm{X}$ & & & $\mathrm{X}$ & & & & \\
\hline Improve Pedestrian Service & $\mathrm{X}$ & $\mathrm{X}$ & & & & & & & & & \\
\hline Improve Bicycle Service & $\mathrm{X}$ & $\mathrm{X}$ & & & & & & & & & \\
\hline Maintain Working Detection & $\mathrm{X}$ & & & $\mathrm{X}$ & & & & $\mathrm{X}$ & & & \\
\hline Maintain Working Preemption & & & & & & & & & $\mathrm{X}$ & & \\
\hline Minimize Pollution and Noise & & & & & $\mathrm{X}$ & $\mathrm{X}$ & & & & & \\
\hline Automate Traffic Counts & $\mathrm{X}$ & & & & & & & & & & \\
\hline Develop Origin-Destination Data & & & & & & & $\mathrm{X}$ & & & & \\
\hline Improve Roadway Safety & & & & & & & & & & $\mathrm{X}$ & $\mathrm{X}$ \\
\hline
\end{tabular}

Table 2.3 Personnel roles in arterial management and opportunities for performance measures to assist and improve tasks, and new roles (highlighted) required for successful deployment of performance measures

\begin{tabular}{|l|c|c|c|c|}
\hline \multirow{2}{*}{ Task } & \multicolumn{3}{|c|}{ Personnel Role } \\
\cline { 2 - 5 } & $\begin{array}{c}\text { Traffic System } \\
\text { Engineer }\end{array}$ & $\begin{array}{c}\text { Traffic Analyst } \\
\text { TMC Operator }\end{array}$ & $\begin{array}{c}\text { Maintenance } \\
\text { Technician }\end{array}$ & IT Specialist \\
\hline Project Management & Assisted & & & \\
\hline System Design & Assisted & & & \\
\hline System Evaluation & Improved & & & \\
\hline Control Plan Development & Improved & & & \\
\hline System Checking & & Improved & & \\
\hline Complaint Handling & Improved & Improved & & Required \\
\hline Maintenance Requests & Improved & Improved & & Required \\
\hline Implementation of Contingencies & Improved & Improved & & \\
\hline Data System Maintenance & & & & Required \\
\hline Data Curation & Required & Required & Assisted & \\
\hline Traffic Equipment Installation & & & Improved & \\
\hline Detection System Maintenance & & & Improved & Improved \\
\hline Communication System Maintenance & & & Improved & \\
\hline $\begin{array}{l}\text { Traffic Equipment Troubleshooting } \\
\text { and Repair }\end{array}$ & & & Assisted & \\
\hline Equipment Inventorying & Assisted & & \\
\hline
\end{tabular}




\subsubsection{Module 2 Overview}

The second module provides more background on the two data sets that are applied for arterial performance measures: vehicle re-identification data, and high-resolution controller event data. Several examples are presented to demonstrate their use. Several examples are included, a few of which are shown here.

Vehicle re-identification data works by matching unique identifiers of vehicles at different locations in a roadway network, to find the travel time between them and other information such as origin-destination patterns. Figure 2.1 shows an overlay chart that includes travel times for one direction through an arterial over a number of different Wednesdays. Vertical lines show when signal timing plans change. The same data is shown as cumulative frequency diagrams for individual Wednesdays in Figure 2.2. The charts illustrate both the overall trend in travel time as well as variation by time of day, and individual dates where travel times were somewhat anomalous.

High-resolution data is used to analyze signal performance in detail, often by using detector states to measure vehicle activity in response to changing phase states. Figure 2.3 shows an example of how vehicle arrivals measured by an advance detector can be related to the overall flow patterns as would be visualized by a time-space diagram. The resulting performance measure, the percent on green, can be tabulated for all of the cycles within a day, as shown in Figure 2.4. The chart shows the individual cycleby-cycle data, which can be highly variable, and a 20-cycle moving average. This type of information can be aggregated to a higher level, and correlated with the travel time data. Additional examples, including a before-after signal retiming study, are presented in the full report.

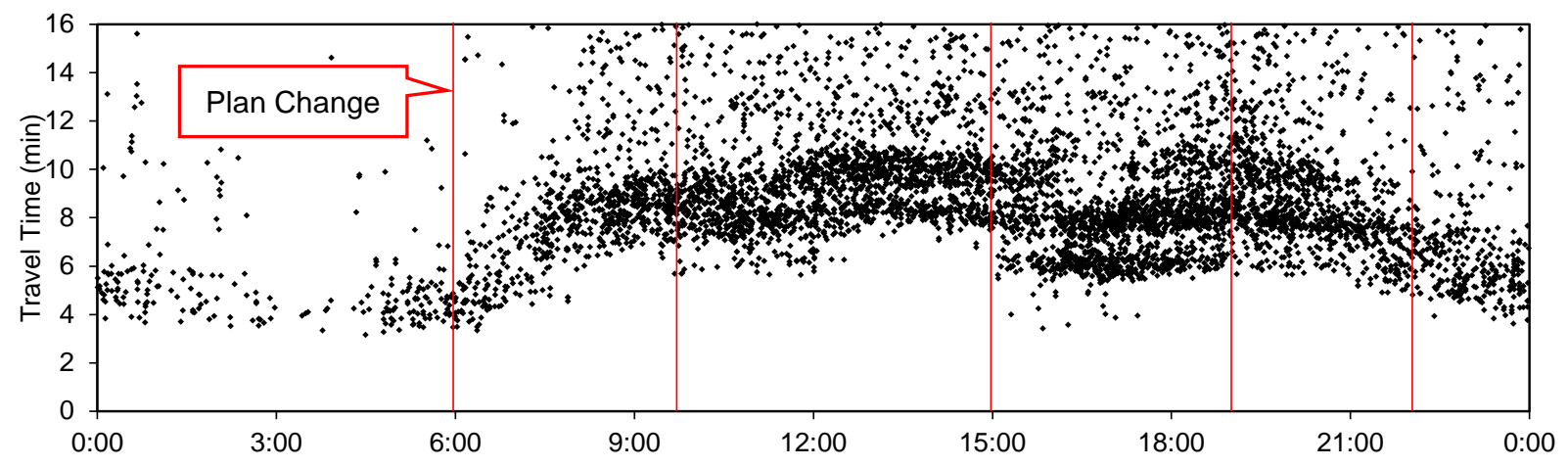

Figure 2.1 View of corridor travel time data aggregated over Wednesdays over 7 months 




Figure 2.2 Cumulative frequency diagrams for individual Wednesdays

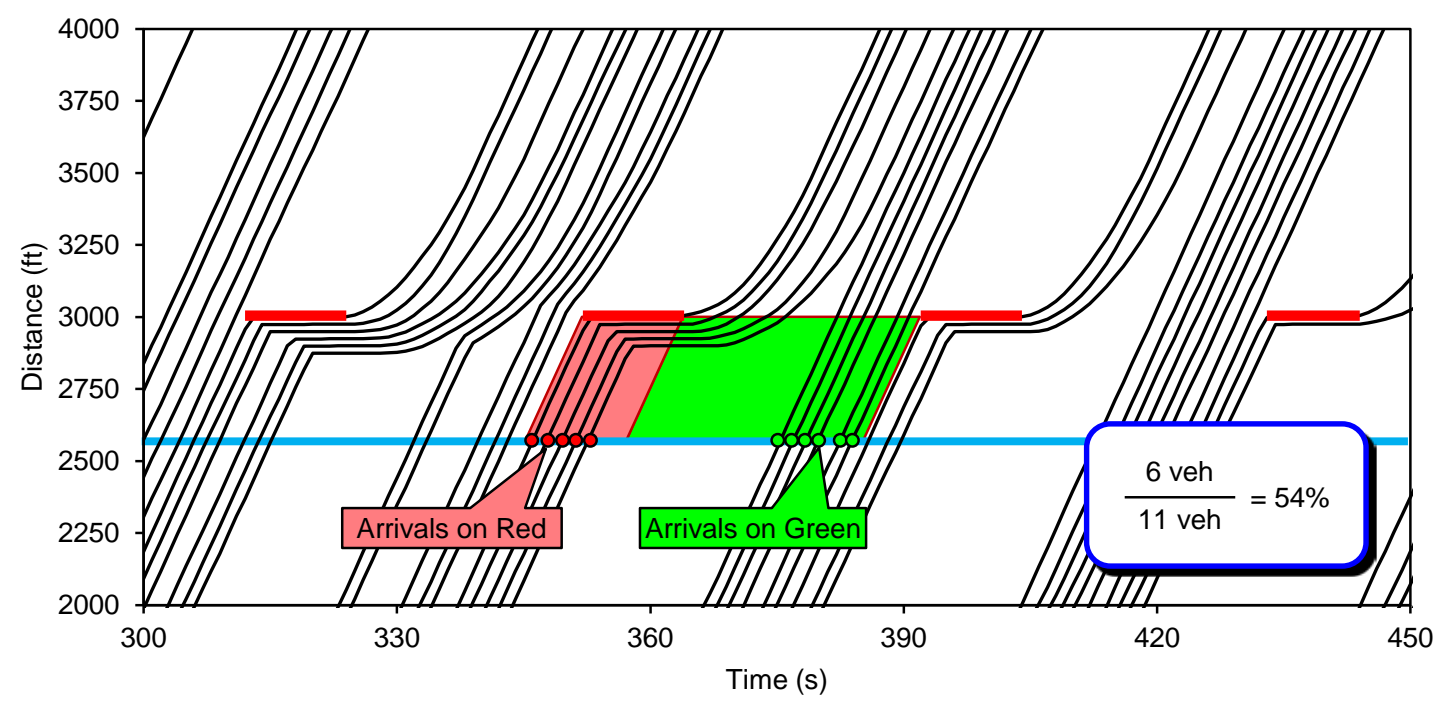

Figure 2.3 An example high-resolution data metric: Percent on green calculation 


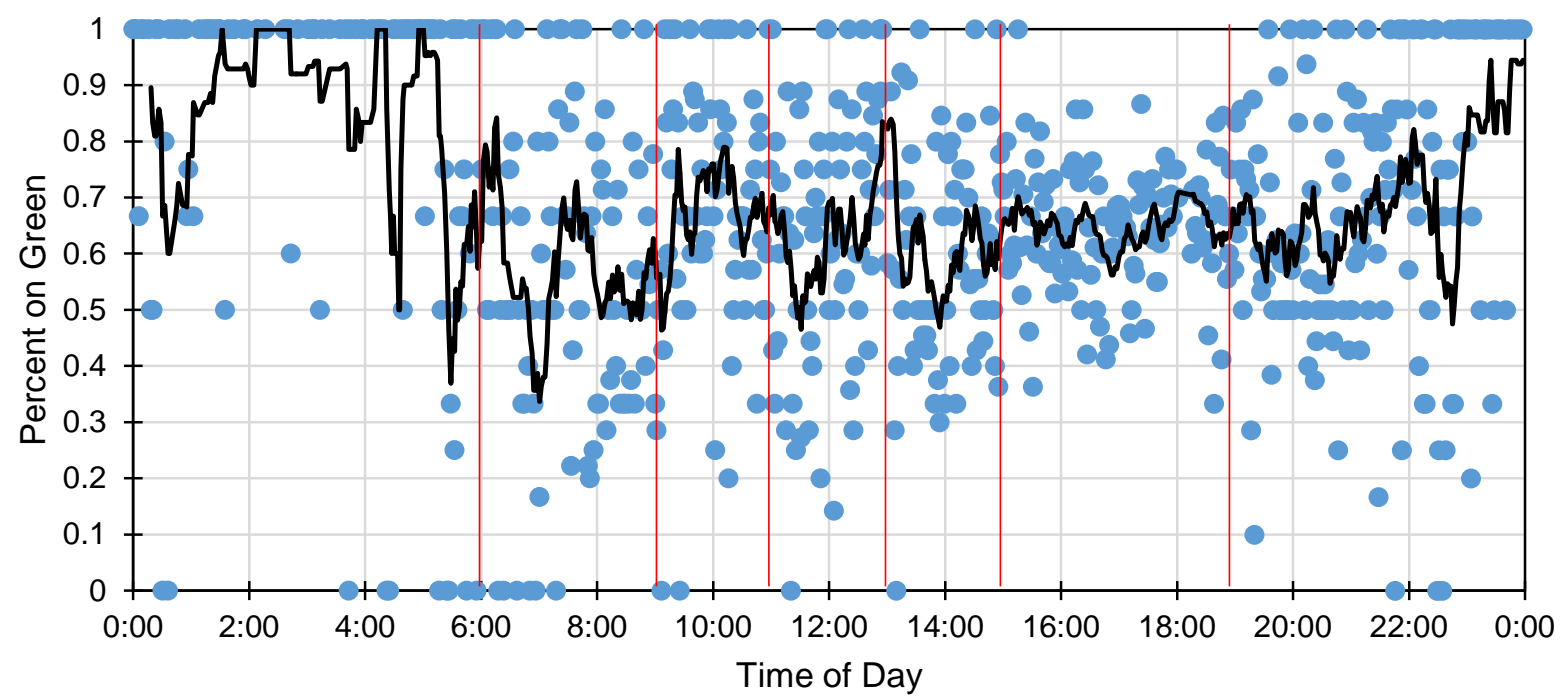

Figure 2.4 An example high-resolution data metric: Percent on green over 24 hours for a movement at a signalize intersection

\subsubsection{Module 3 Overview}

This module looks at how vehicle re-identification data and high-resolution controller event data can be applied to travel time and travel time reliability objectives. These are most relevant to signal progression through routes in a network, typically following corridors, and where arriving traffic interacts with signal timing at individual approaches of individual intersections. There are clearly opportunities for analysis at the intersection, corridor, and network level. Module 3 presents several examples from case studies where the data was applied to signal progression objectives.

The Purdue Coordination Diagram (PCD) is explained in detail in this module, and a number of observable traffic conditions are related to how they would be exhibited in the PCD through several visual examples. For a simple, contrasting illustration, consider good progression versus poor progression. Figure 2.5a shows a time-space diagram exemplifying perfect progression, with no vehicles stopped or slowed by a traffic signal, and Figure 2.5b shows a PCD from real-world data that exhibits a similar pattern, with most of the vehicles arriving in green during certain times of day. Meanwhile, Figure 2.6a shows a different pattern, with many vehicles arriving in red, and Figure 2.6b shows a corresponding PCD where most arrivals are in red during certain times of day.

Figure 2.7 presents an example of corridor travel times, which are plotted as monthly averages for weekdays over the course of a year. The red line shows when signals were retimed on the corridor, which corresponds to a visible decrease in the travel times. These are charted over time, showing that the improvement is sustained for at least a year. This demonstrates an effective corridor level metric, in this case supporting a before-after review as well as following up with continuous monitoring. 
Performance characteristics of intersections and corridors can also be aggregated to the network level to identify deficiencies across the system. Figure 2.8 shows an example using a performance measure called the maximum vehicle delay (MVD), which looks at the longest possible vehicle delays by movement at an intersection. This map view shows the highest MVD value by intersection across seven corridors in the Indianapolis area. The data are sorted by quintiles, enabling the locations of the greatest delay to be quickly identified.

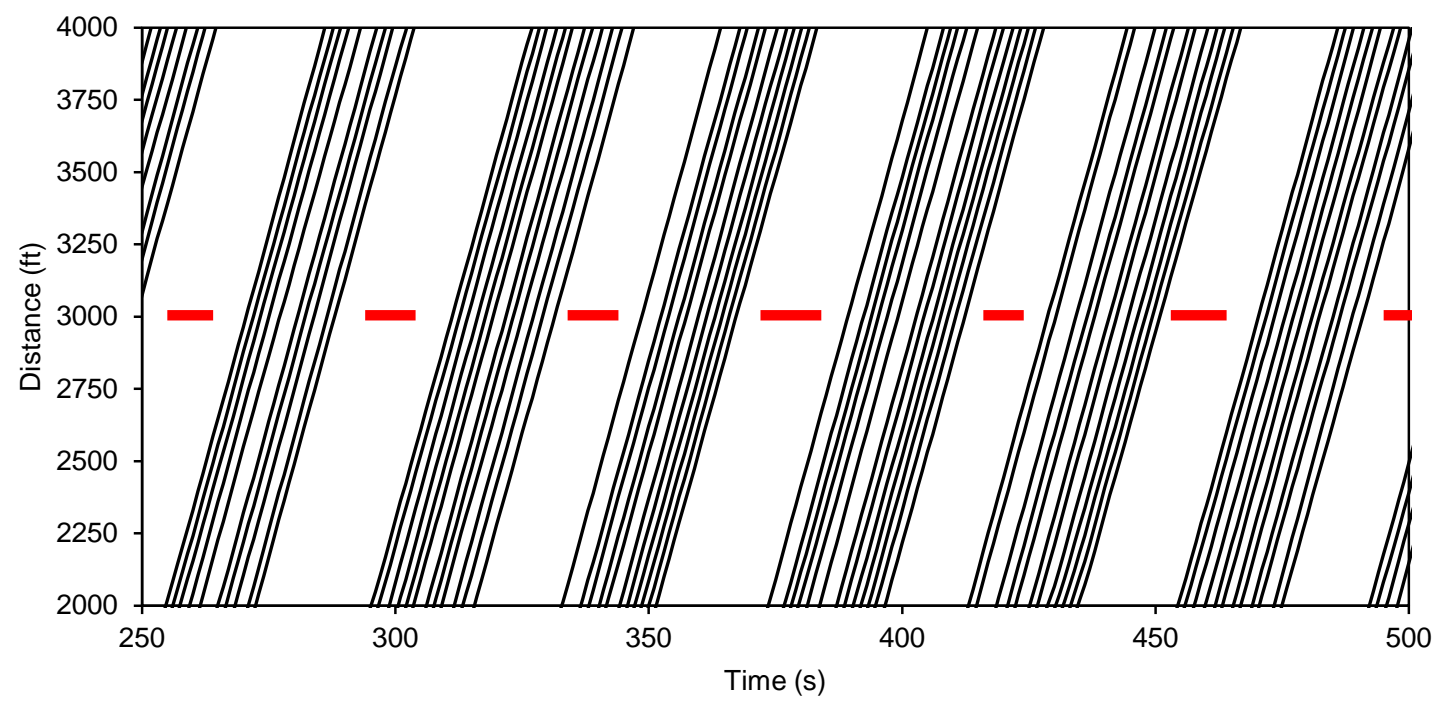

(a) Conceptual time-space diagram

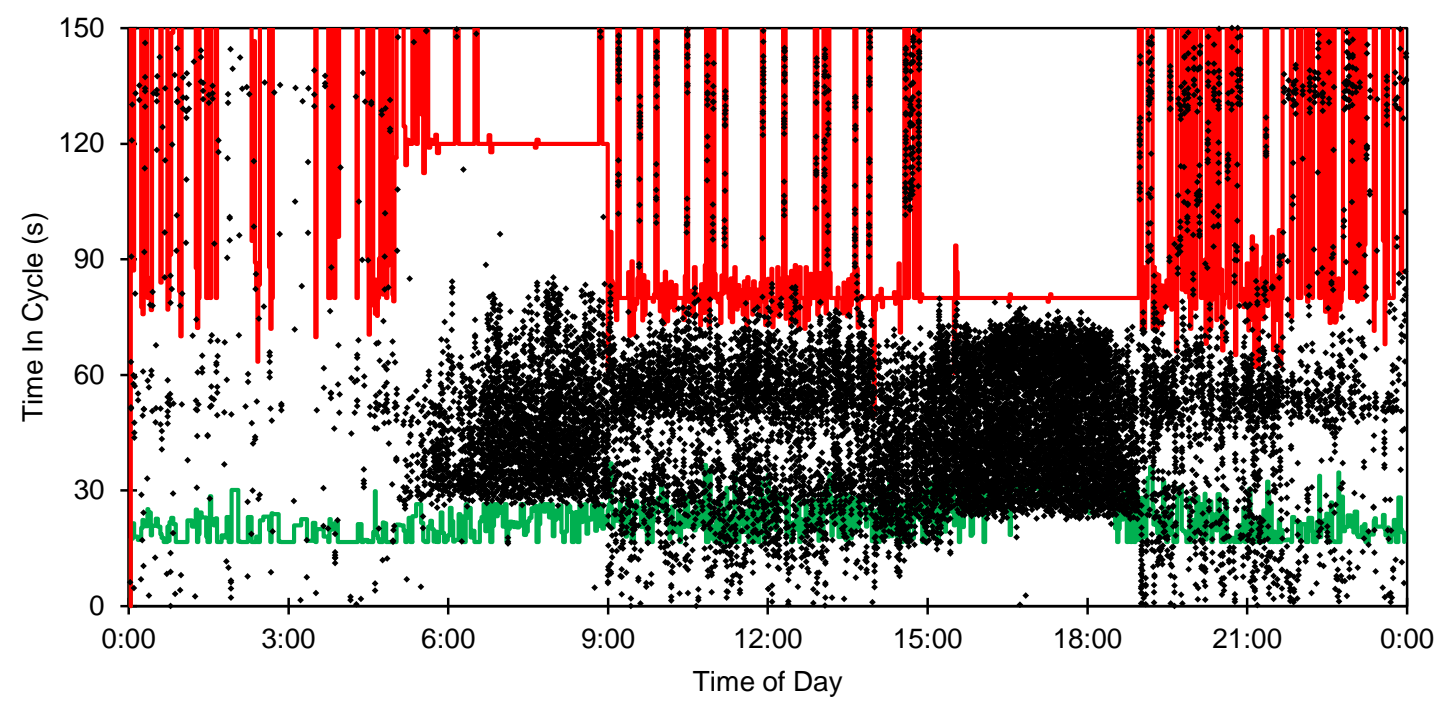

(b) Coordination diagram

Figure 2.5 Traffic flow through an intersection with near-perfect progression 


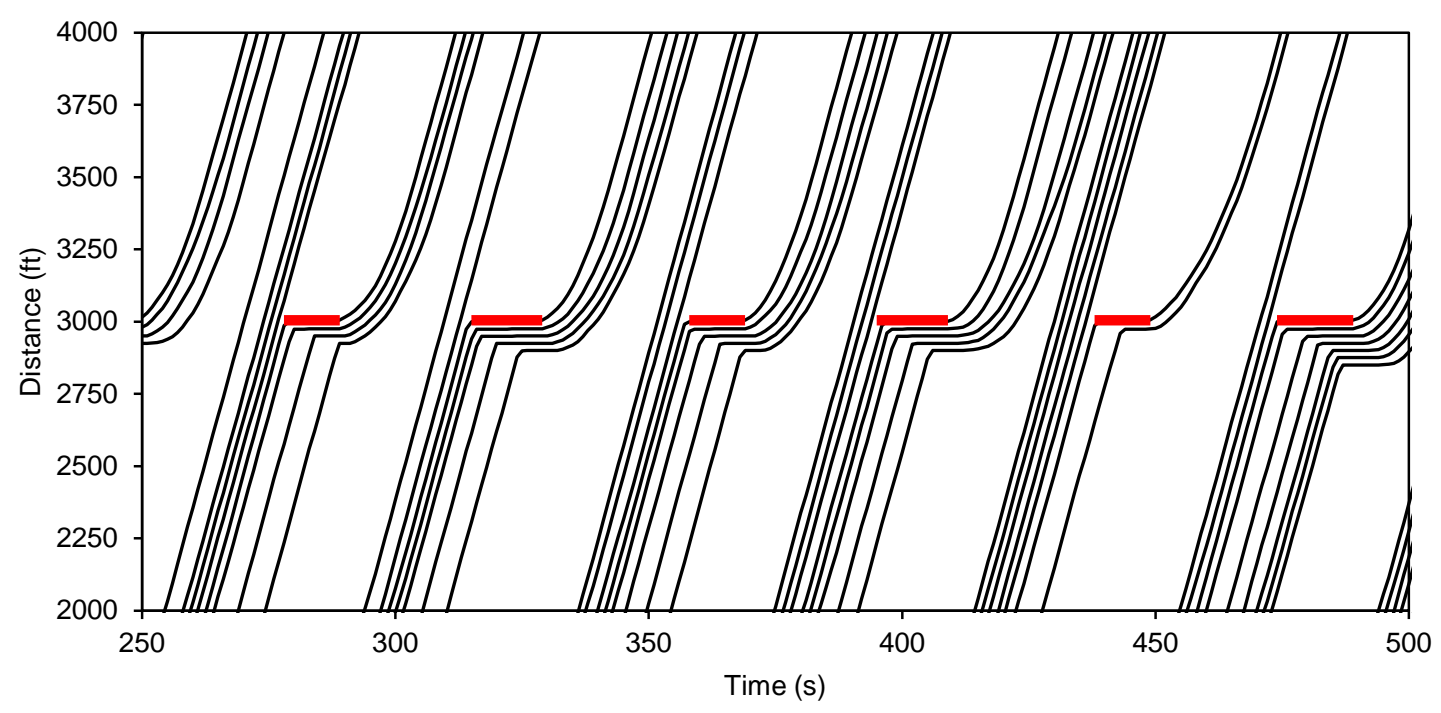

(a) Conceptual time-space diagram

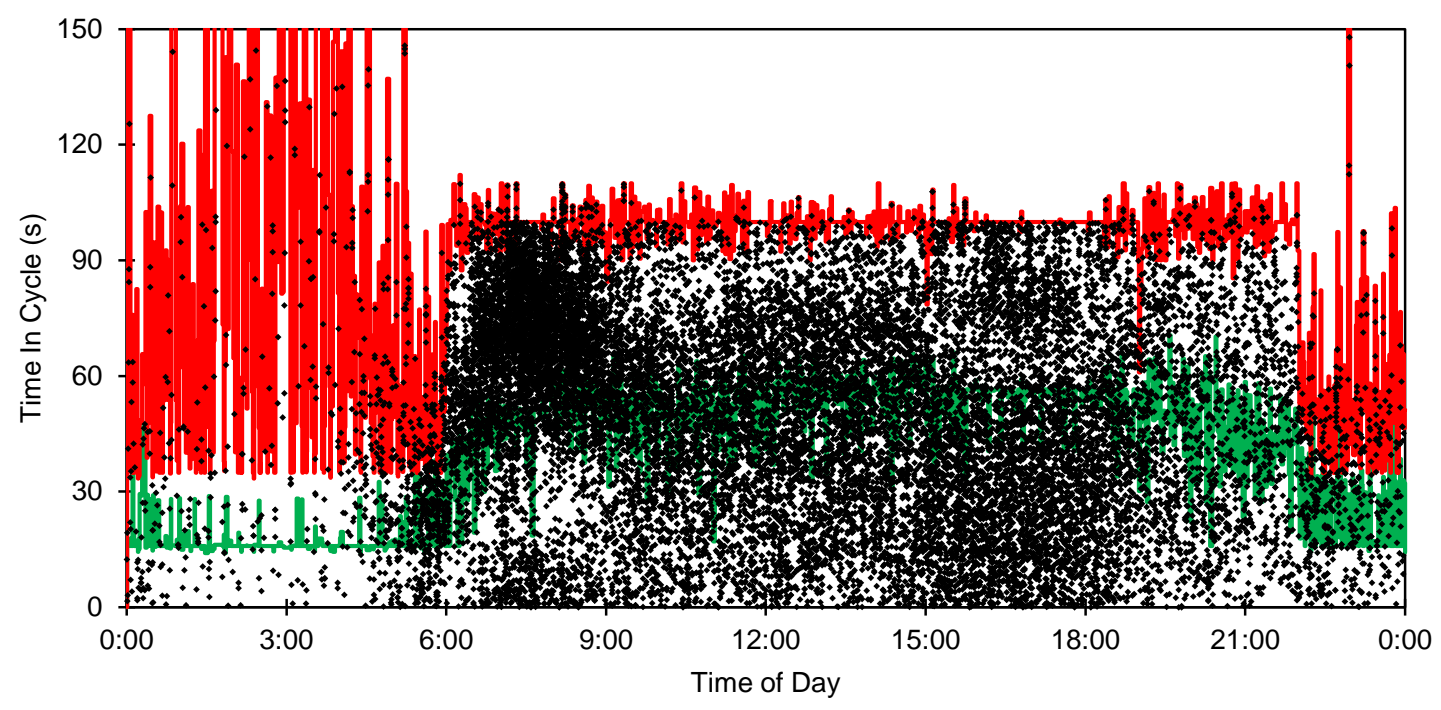

(b) Coordination diagram

Figure 2.6 Traffic flow through an intersection with poor progression 


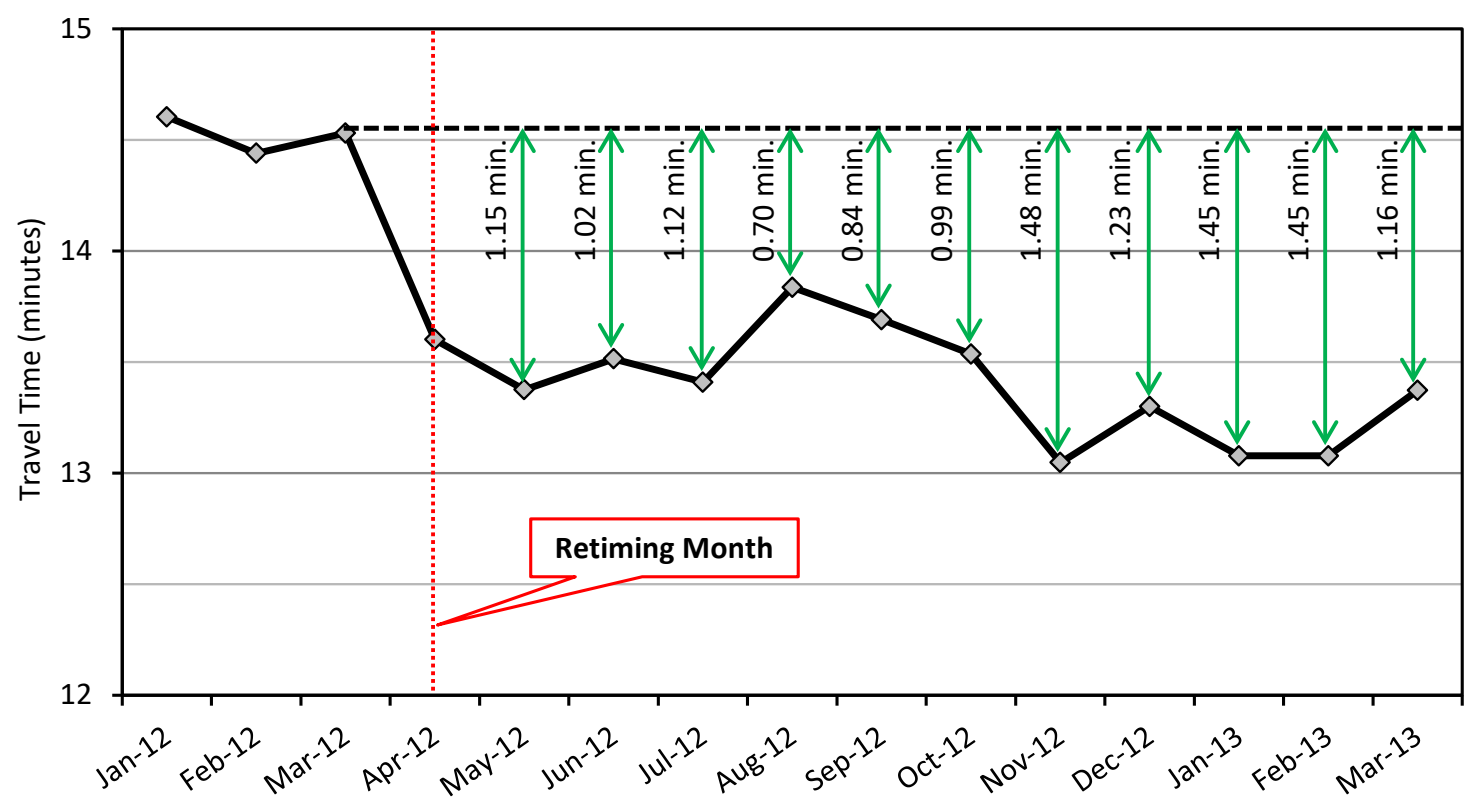

Figure 2.7 Evaluation of corridor travel times over one year after signal retiming

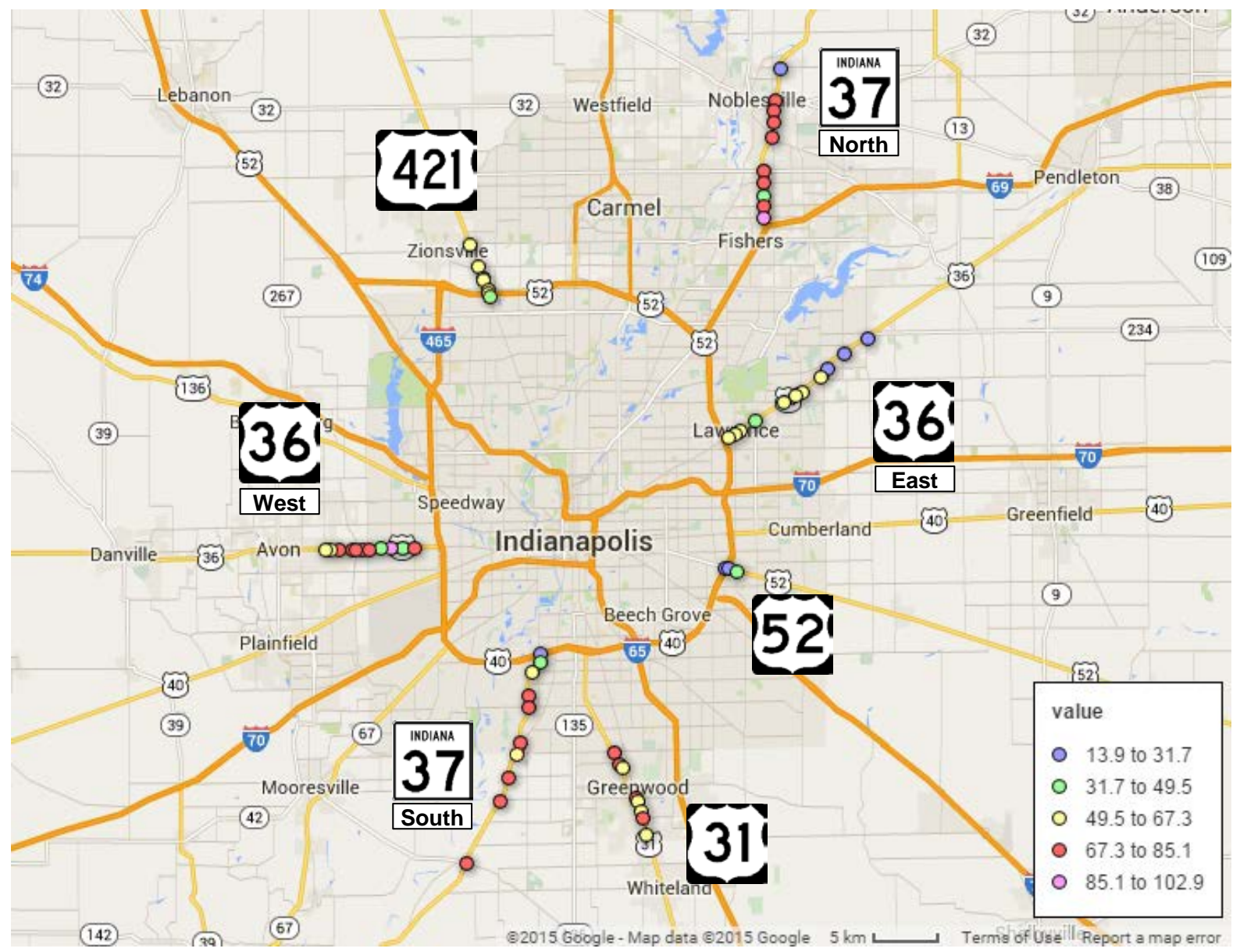

Figure 2.8 Map showing highest median MVD by intersection, organized into five groups

Final Report 


\subsubsection{Module 4 Overview}

Module 4 examines performance measures relevant to traffic demand and intersection capacity in the analysis of arterial networks. Several performance measures are demonstrated in a series of use cases that show application at the intersection, corridor, and network levels. This module demonstrates the performance measures using several case studies that investigate impacts of changes to the signal timing, including both split adjustments and cycle length adjustments. Applications of the data for predicted impacts of traffic growth are also discussed.

For an individual intersection, the performance of individual movements can be examined. Many intersections use an eight-phase signal timing scheme that separates left-turn and through movements, as shown in Figure 2.9. This structure can be directly applied as an organizational pattern for viewing movement performance. For example, Figure 2.10 shows the number of split failures per movement/phase, which maps directly to Figure 2.9. A split failure is an instance where there is insufficient green time to clear the amount of demand present on a phase within one cycle. The chart quickly reveals which movements are experiencing the highest number of these, and during what times of day they are occurring. Figure 2.11 shows a similar view for a before-after study showing how the number of split failures changed with respect to a timing plan change.

Movement values can easily be aggregated to the intersection, corridor, and network levels. Figure 2.12 presents an example taken from the network level. In this case the data is abstracted away from the geographic relationships and the intersections are ranked by performance from the highest to smallest number of split failures per hour, for different times of day. Here, the maximum number of split failures on any individual movement is used as the individual intersection value. Perhaps surprisingly, this analysis finds that the midday time period actually experiences more split failures than the PM peak for most of the system, and the AM peak has fewer split failures than the evening time period. Another interesting observation is that the resulting curve shapes are roughly consistent from one time of day to the next, and the shapes suggest a Pareto-like distribution. That is, a relatively small proportion of the intersections would seem to account for a disproportionate number of the split failures.

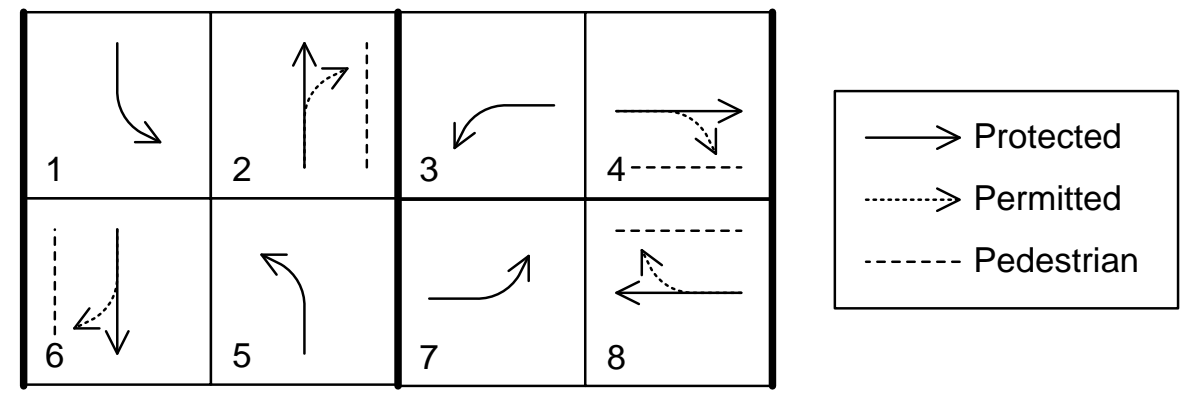

Figure 2.9 Ring diagram showing the sequence of phases at a signalized intersection 

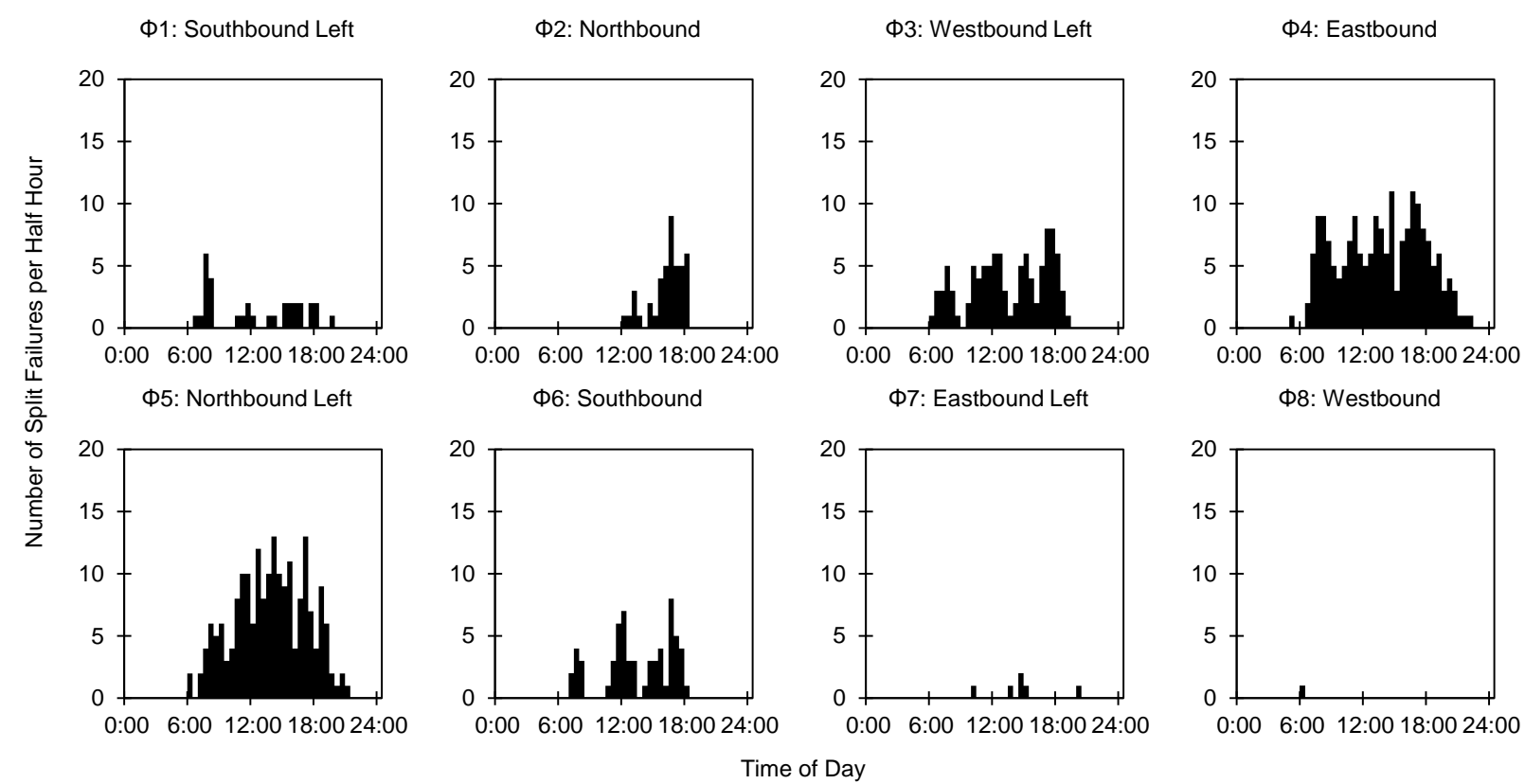

Figure 2.10 Number of split failures per half-hour, by phase


Figure 2.11 Number of split failures before and after split adjustment 


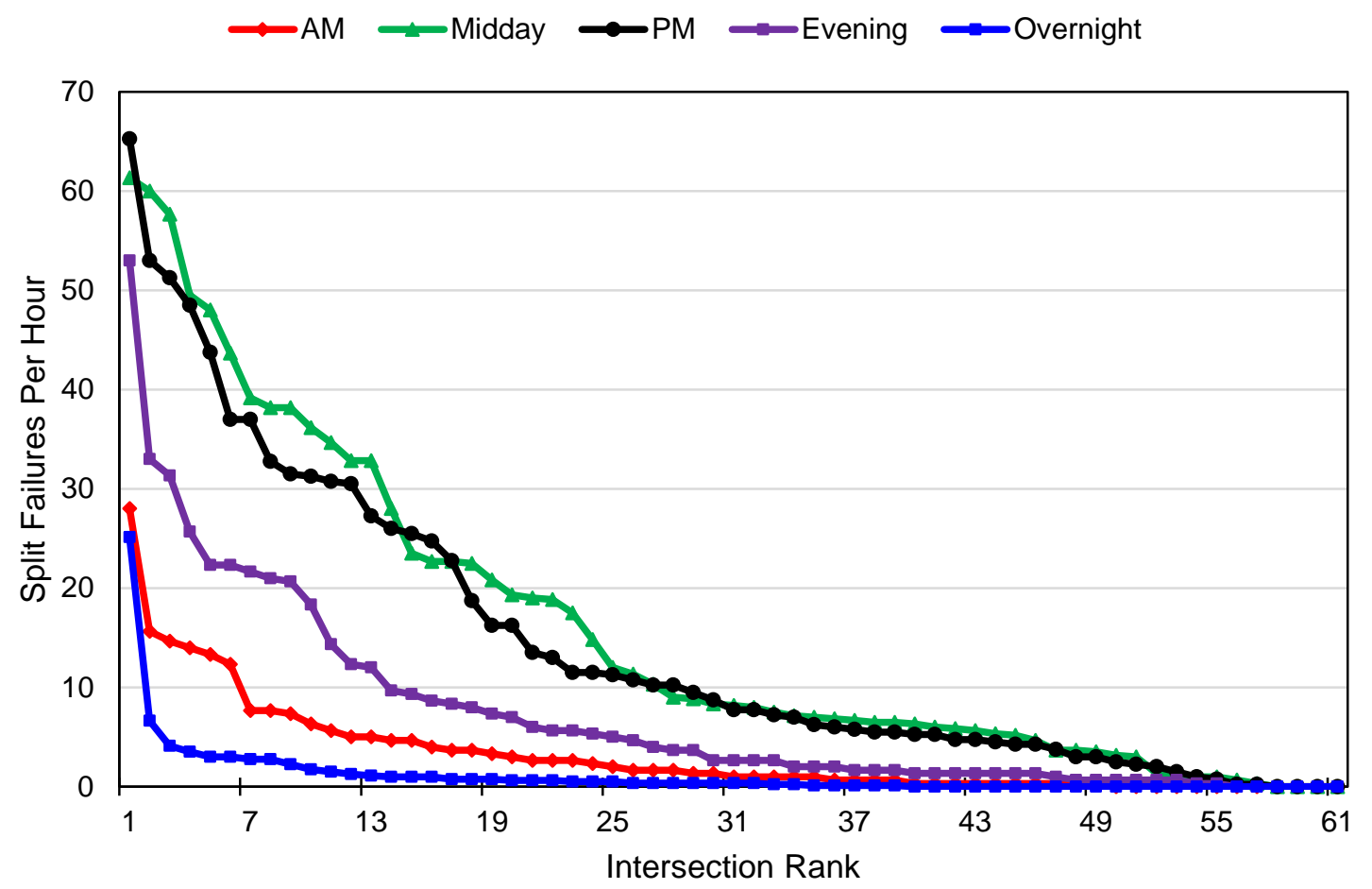

Figure 2.12 Maximum phase failure rate per intersection across 61 intersections, by time of day

\subsection{Real-Time Report}

The previously described four-module report introduces a variety of performance measures for arterial analysis with a temporal perspective of the recent past, or with comparisons of before and after periods. It is also necessary to develop methods of viewing current conditions in real time. This second report focuses on possible ways to use of vehicle re-identification data and high-resolution data for real-time analysis.

Real-time analysis enables system operators in a traffic management center (TMC) to respond to conditions as they develop and intervene when needed. Sensors have been used for real-time condition reporting on freeway systems, but these methods do not translate well to arterial conditions because of the different nature of interrupted flow facilities. Essentially, that speed measurements on such arterial roadways do not correlate with travel times because the delay is incurred by stopping and queuing at intersections, rather than with speed reductions due to congestion. Furthermore, it is not possible to measure queuing activity using the same spatial and temporal distribution of speed measurements that can be used for freeways.

The first chapter of the report examines system requirements for real-time analysis. The system needs are explored through discussion of a scenario where freeway traffic is diverted onto parallel arterial highways. Basic questions, as would be asked of system operators in such a scenario, are outlined that represent what needs to be ascertained from data. For example, the operators must determine an 
appropriate detour route, determine if signal timing needs to be changed to accommodate the detour traffic, provide an anticipated travel time to travelers, and so forth.

The next chapter investigates possible uses of vehicle re-identification data for real-time analysis of arterial performance. The proposed approach is to look at a record of past data to provide context for the current operation, using the concept of cascading windows. Figure 2.13a presents an example of cascading windows on top of example travel time data. Starting after 6:15 a.m., 15-minute windows are created for analysis, which move forward in time by a 5-minute step. Combining the analyses for the series of windows provides context for the current travel times. Three types of visualizations are proposed in this report: cascading CFDs (Figure 2.13b), cascading histograms (Figure 2.13c), and stacked histograms (Figure 2.13d). Each of these shows the relevant data for the current conditions superimposed on the past conditions from previous time windows. The cascading CFDs show that travel times are increasing, because the current CFD is to the right of the previous ones. The slope is also becoming less steep, meaning that there is increased variability of travel times. The cascading histograms show the same trends with a different graphical interpretation: the peak of the current histogram moving to the right and spreading out. Finally, the stacked histogram shows an overall trend from all the data, but the contribution from the current time window (the darkest blue series) is clustered toward the higher end of the overall distribution, and is fairly spread out. These visualizations enable the analyst to determine if travel times are increasing or decreasing, or becoming more or less reliable. 


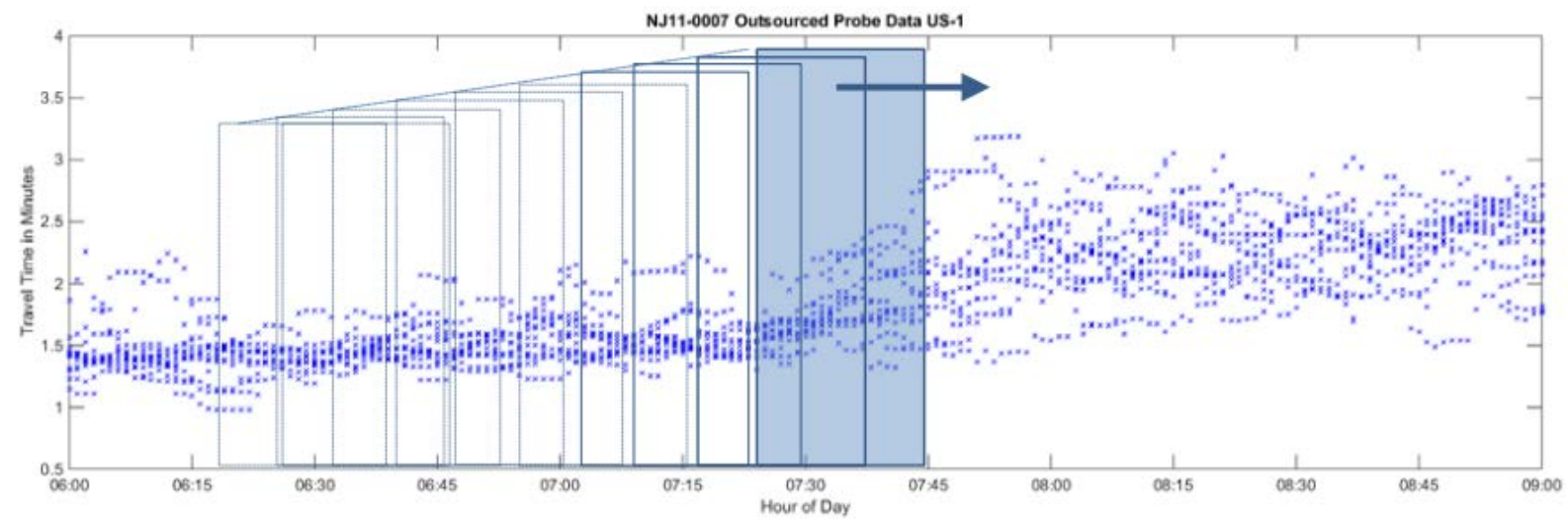

(a) Cascading time window concept.

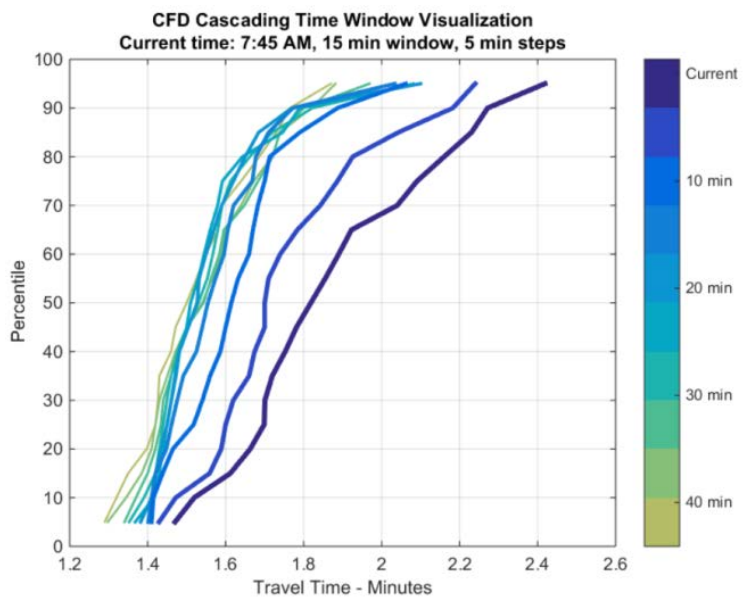

(b) Cascading CFDs

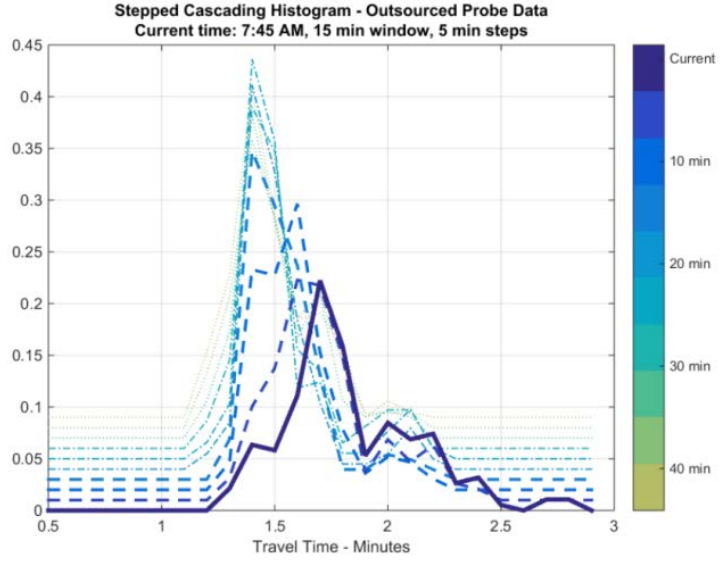

(c) Cascading Histograms

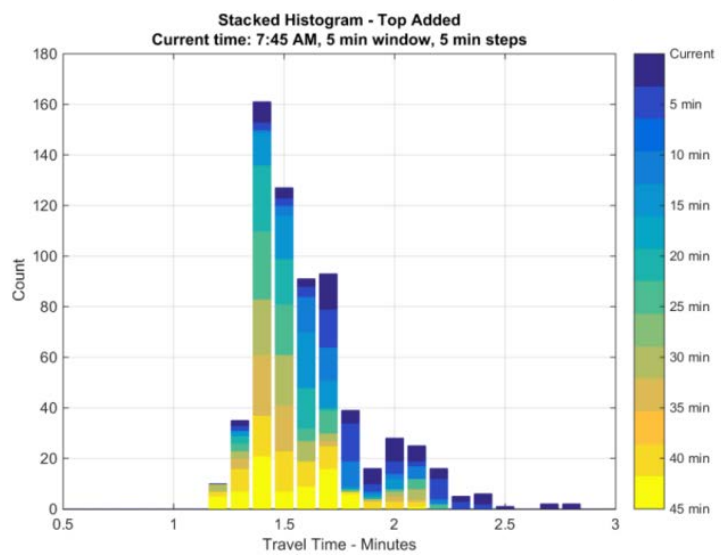

(d) Stacked Histograms

Figure 2.13 Visualizations of travel time for real-time monitoring, using cascading windows 
The technical considerations for aggregating data are further discussed in the next chapter of the report. Signal operation introduces some unique requirements for data collection. The choice of the size of a time window is influenced by the cycle lengths used by the traffic signals. Cycle lengths typically range between 1 and 3 minutes; an aggregation window needs to be longer than one cycle length, and it is preferred to include several cycles. Care must also be taken to avoid the choice of a time window that interacts with the cycle length in such a way to influence the sampling. A minimum time window of 10 minutes is suggested to avoid these issues. Another technical consideration is that of data density. Based on analysis of resulting graphics with diminishing sample sizes, a minimum of 15 samples is suggested. Other considerations such as the number of steps are also examined.

The final chapter in the report discusses potential uses of the high-resolution data for real-time analysis. Two performance measures highlighted for this application are the use of the green occupancy ratio (GOR) and red occupancy ratio (ROR) to examine excessive demand. As discussed earlier, high values of ROR and GOR are indicators of split failures, and successive split failures indicate that queues are likely growing. Figure 2.14 shows an example view of such a metric, where each approach at an intersection shows the number of split failures occurring over a sample time period (such as the past 30 minutes). In this example, it is likely that the northbound approach has an operational problem. Traffic volumes are another piece of information that could potentially be obtained from high-resolution data in real time.

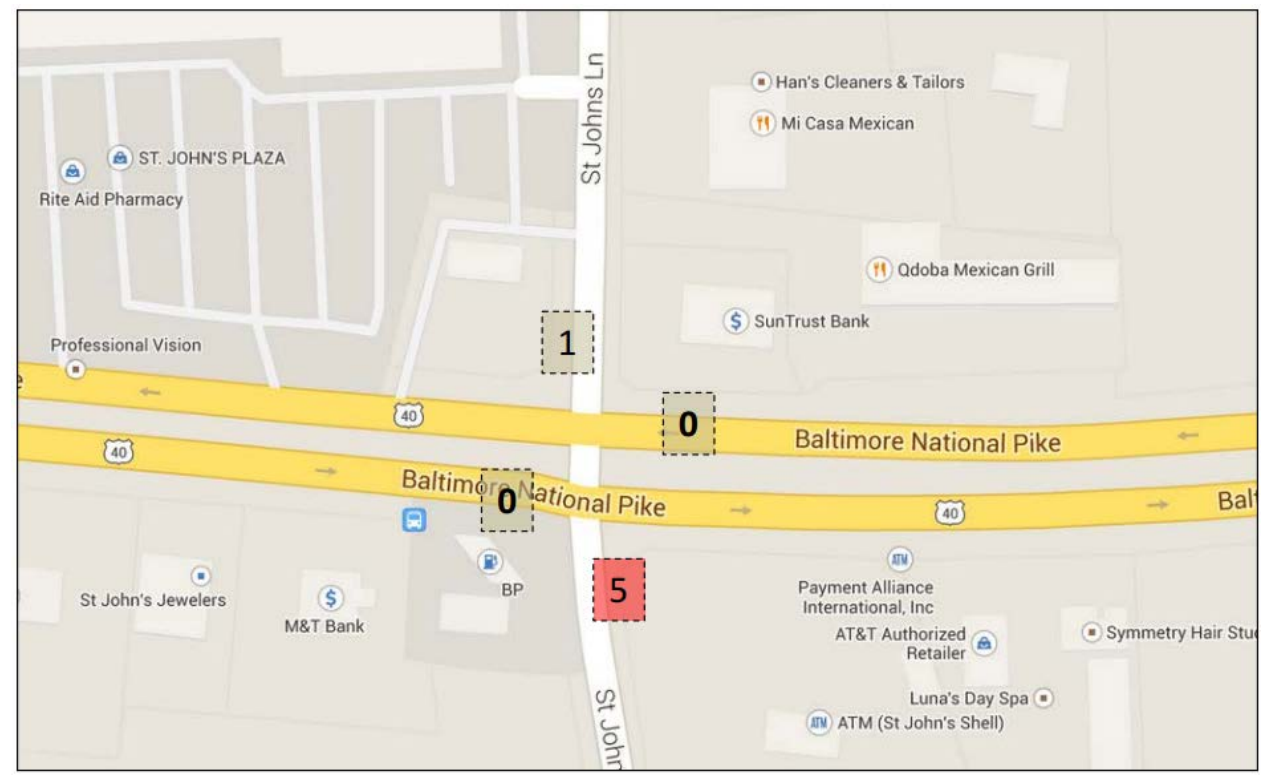

Figure 2.14 Proposed visualization of the number of split failures on approaches at a signalized intersection 


\subsection{Arterial Trip Length Characteristics Report}

Methods to classify where a roadway falls on the mobility versus land access spectrum have traditionally relied on professional judgment typically based on assessing its geometric attributes. This research takes an alternative approach to quantifying the throughput versus land access properties of a roadway by introducing a new method called the "Roadway Trip Length Characteristic" or RTLC. Rather than using indirect attributes of the roadway design, such as intersection density or number of access points, the RTLC directly measures how travelers use the roadway for trip making purposes. Specifically, in this approach, the distribution of trip distance along a corridor is used as a quantifiable indicator of throughput versus land access continuum. A preponderance of long trips along a corridor would indicate the primary function of throughput. If the majority of trips along the corridor are short in duration, it would indicate that vehicles are accessing goods and services within the corridor. The full distribution of trip lengths (defined as the RTLC) reflects the mixture of conflicting demands on the corridor, creating a defining signature. This RTLC can be specified under various conditions such as time of day (rush hour versus non-rush hour), special events, or seasonal conditions.

Measuring roadway trip lengths and the statistical distribution of those trip lengths on a specific facility can be performed cost effectively with modern re-identification equipment The proliferation of consumer wireless technology allows re-identification to be performed automatically using unique identifiers in Bluetooth and Wi-Fi electronic communication protocols.

Bluetooth and Wi-Fi re-identification sensors, placed at key intersections along a corridor as illustrated in Figure 2.15, record the unique electronic identifiers of consumer electronics in vehicles as they pass. A roadway vehicle trip is described by the series of sensors traversed. A vehicle that enters the roadway at $3^{\text {rd }}$ street and exits at $10^{\text {th }}$ street will be observed by sensors $\mathrm{C}, \mathrm{D}, \mathrm{E}$, and F, producing a sensor sequence pattern of CDEF. By analyzing these patterns, and with knowledge of the distance between each sensor, trip lengths can be measured and various statistics about the trip length on the roadway can be calculated.

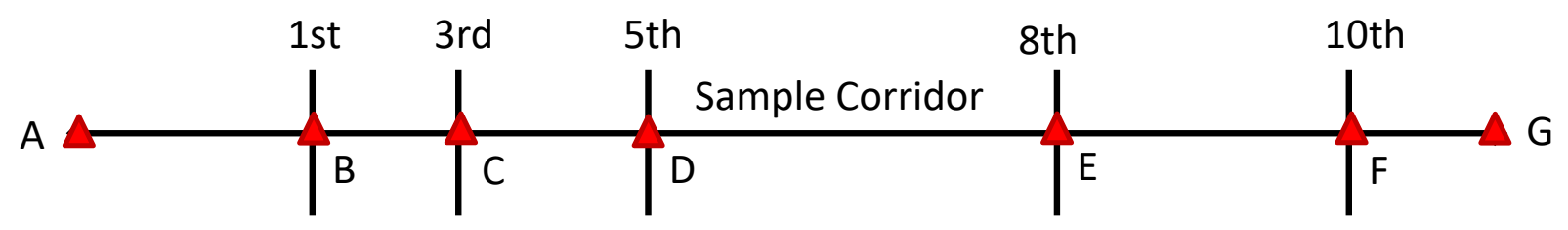

Figure 2.15 Placement of re-identification sensors for assessing corridor movement

The case study from Maryland route 140, depicted in Figure 2.16, illustrates the use of the RTLC approach. Sensors were placed along MD-140 for a period of approximately two weeks, beginning on June 5, 2014 and extending to June 17, 2014.

Trip sequences were assessed for all Bluetooth re-identified vehicles in this corridor during the data collection period. Bluetooth is able to sample approximately $5 \%$ of all trips. Combining the trip sequencing information with the distance between sensors provides the base data to illustrate the distribution of roadway vehicle trip lengths. A histogram of observed roadway vehicle trip lengths is illustrated in Figure 2.17.

Final Report

Contract \#: DTFH61-14-C-00035 


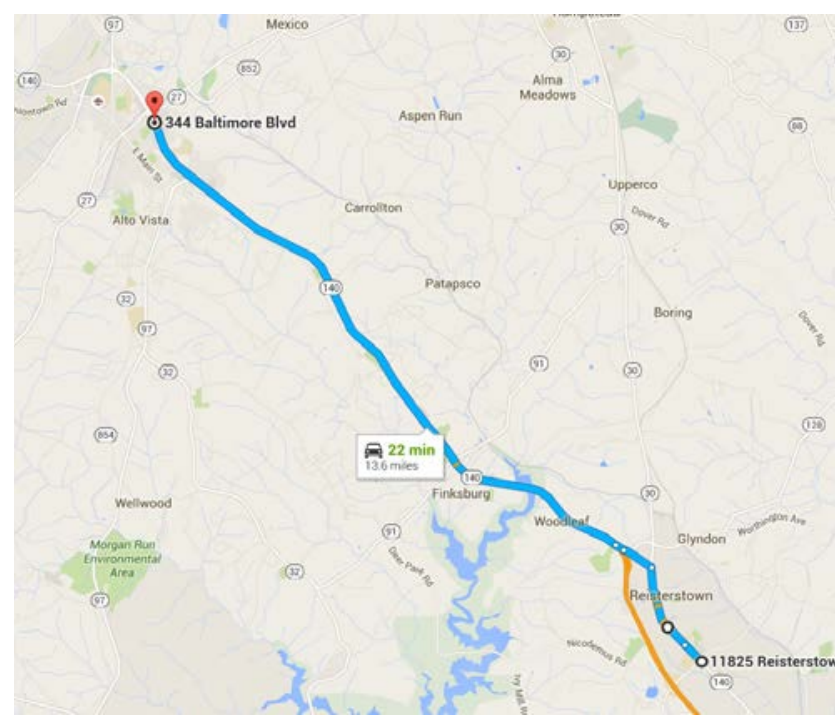

(a) Location of the MD 140 study corridor.

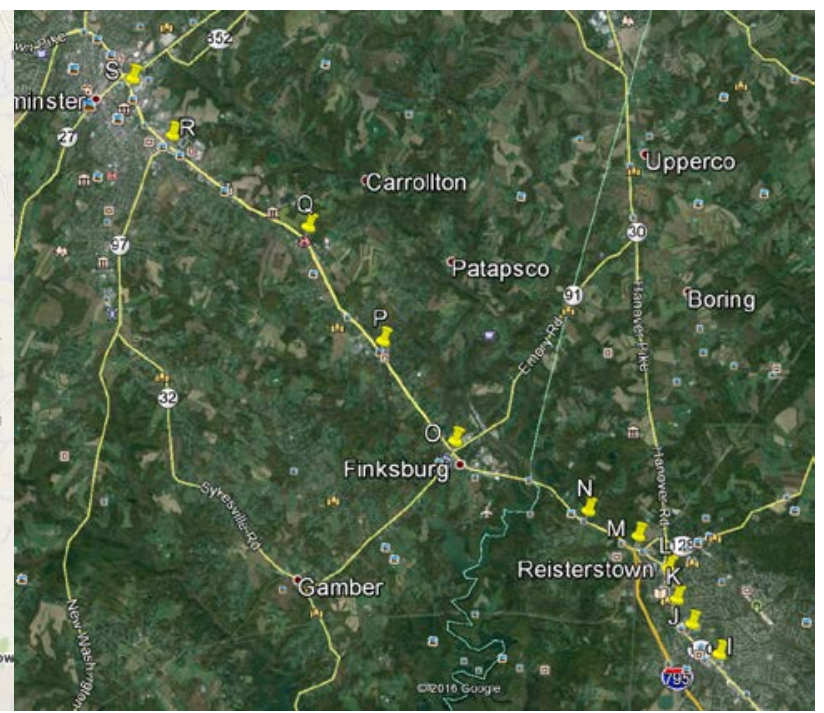

(b) Placement of re-identification sensors.

Figure 2.16 Location for the arterial trip length characterization study

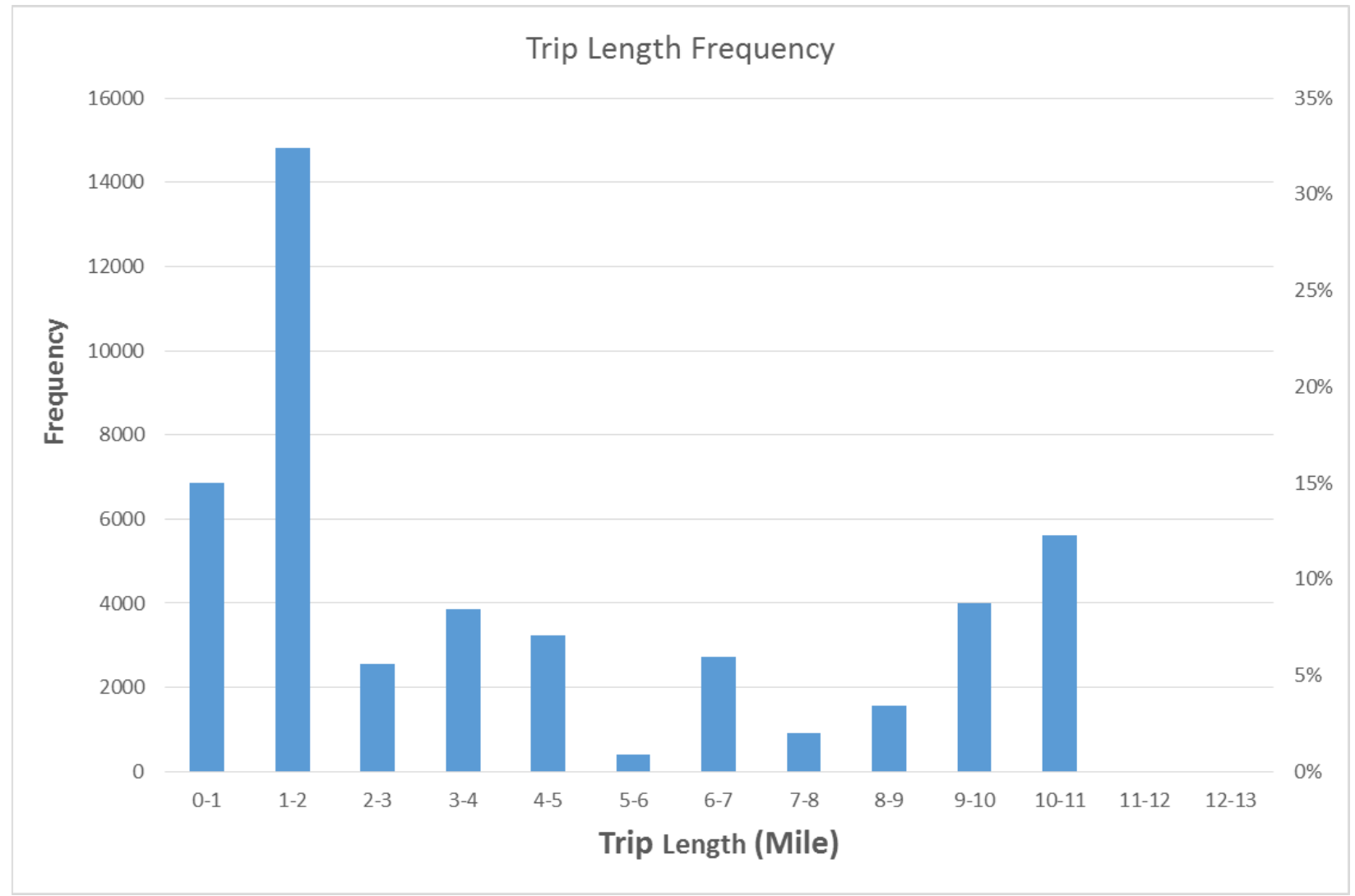

Figure 2.17 Histogram of observed trip length frequency for MD-140 
The roadway trip length statistic is an objective measure directly reflecting the subjective concept of throughput versus land access. As a directly measurable attribute, it is useful for engineering and performance assessment processes. It can supplant subjective judgment or indirect measures to provide a repeatable and defendable method for classifying roadways in order to compare and contrast roadway operations. Moreover, it can also be used to find like roadways across broad geographical regions to compare for performance, as illustrated in Figure 2.18 below. Figure 2.18 contrasts the RTLC from MD140 with that obtained from a segment on New Jersey route 37. In this comparison, the RTLC is illustrated (and normalized) using cumulative frequency diagrams (CFD) such that the distribution of trip lengths can be directly compared. Whereas the through movements were a dominant use of Maryland Route 140, in contrast, the trip lengths on NJ37 are more evenly distributed across multiple trip lengths.

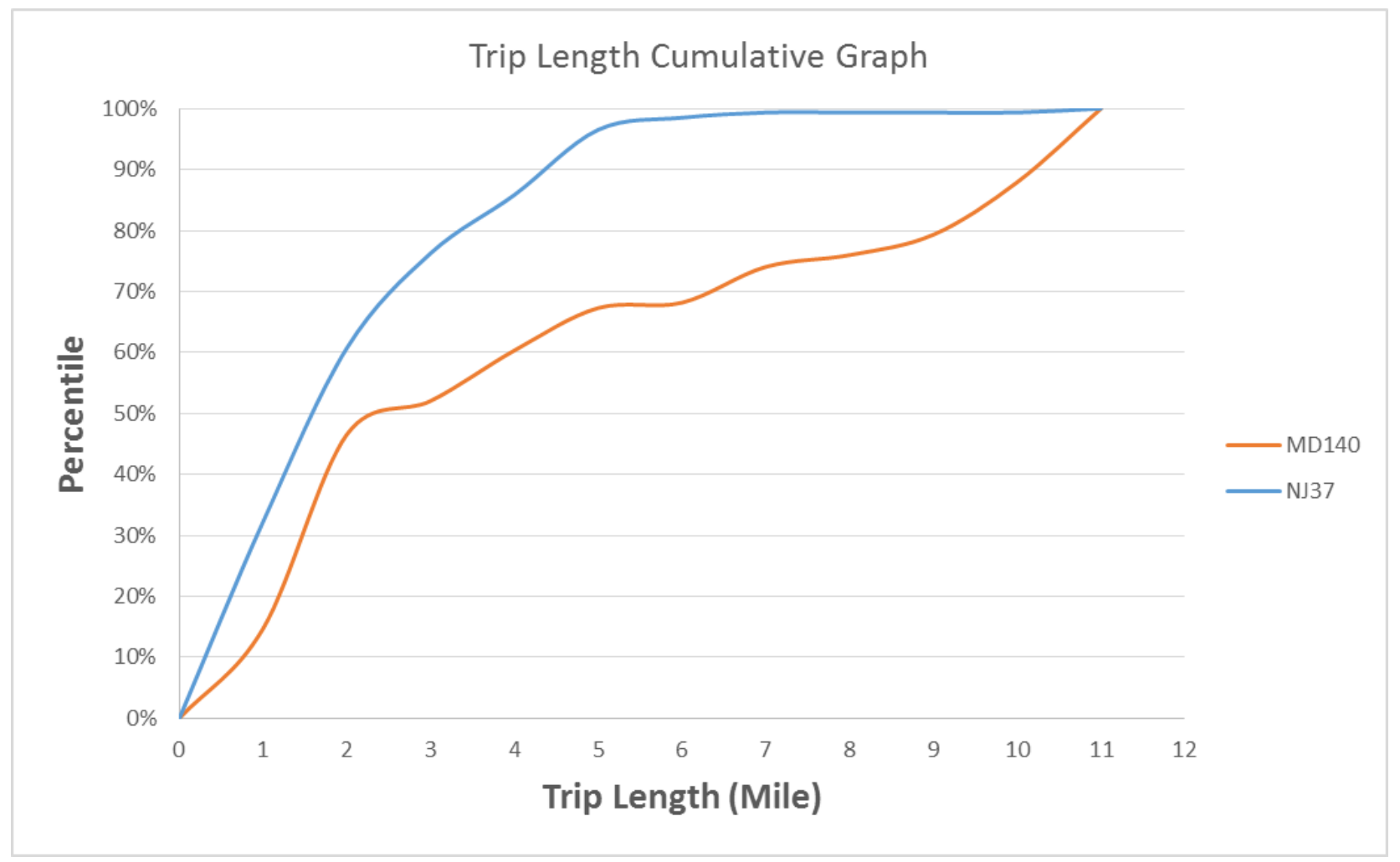

Figure 2.18 Cumulative frequency diagram of trip lengths from two different corridors 
Data and inferences gained from the RTLC method allow for comparisons of vehicle traffic operations either on the same facility (different times of day) or between two different facilities. This provides a valuable tool for management policy, signal timing and coordination schemes, and other operations treatments.

Although the RTLC was illustrated with Bluetooth based re-identification data, the RTLC is not restricted to any particular type of re-identification data, nor is it only applicable to re-identification data. As modern data sources continue to mature, individual vehicle location data can also be used as the basis for calculating RTLC information. Data sources such as vehicle probe based traffic data and vehicle-tovehicle dedicated short-range communication are anticipated to provide such data in the future.

The RTLC method provides a repeatable and objective procedure to characterize roadway function based on how it is used by drivers. The RTLC also complements classification by design attributes and allows for a higher granularity approach to roadway characterization.

\section{$2.4 \quad$ Network Report}

As mentioned in Chapter 1, the granular performance measures need to be aggregated up to higher levels to assist managers and decision makers with a way to quickly assess the entire system. This high level view is explored in the network report.

The report begins by examining concepts in pavement management systems, where performance based management is a mature practice. Figure 2.19 shows two key concepts. One is the assessment of facilities with different criteria, which can then be used to develop an aggregate score, as illustrated in Figure 2.19a. Here, a " 1 " rating is good and " 3 " is poor; four different aspects of pavement performance are rated for different facilities. With this, one can begin tracking performance over time. Figure 2.19b shows the percentage of facilities with pavement in good condition and the percentage in poor condition over time. The chart reveals gradual improvement.

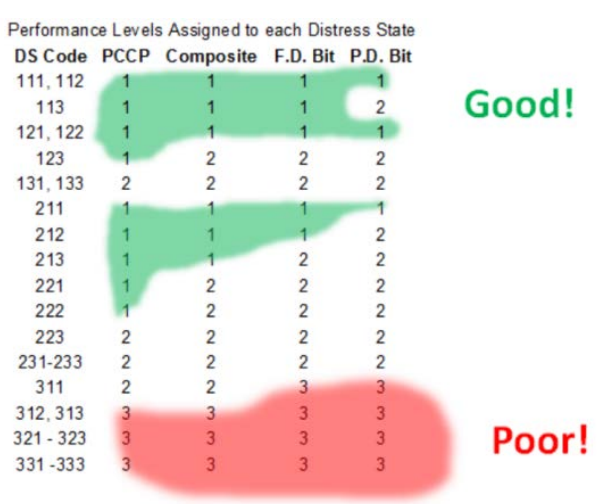

(a) Condition ratings for different combination of performance measures.

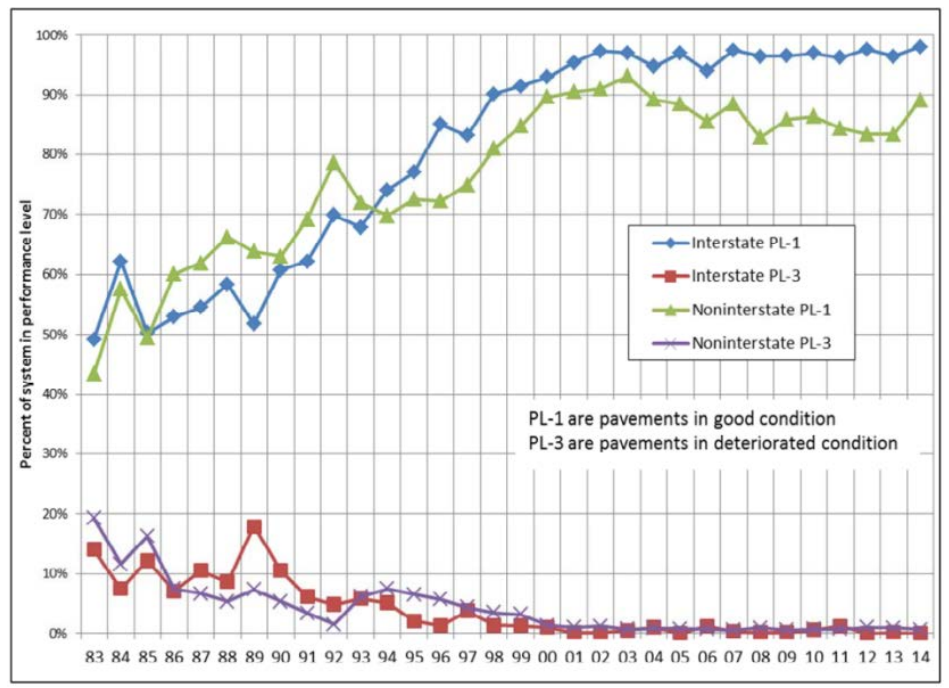

(b) Condition rating over time from the Kansas State Highway System.

Figure 2.19 Condition rating concepts from pavement management 
This overall management concept is applied to arterial traffic operations in the network report. The first step is to classify and segment arterials by their attributes. The reason for doing so is to identify target metrics appropriate to the roadway class. It would not make sense, for example, to use the same condition thresholds for a higher-speed rural arterial with a lower-speed urban arterial. The second chapter of the report discusses potential properties of arterial highway segments that could be used for this purpose, referring to the AASHTO Green Book and Highway Capacity Manual for reference on design categories and functional categories.

The third chapter proposes a series of performance measures and target thresholds to serve as the rubric for arterial condition ratings. The median travel time, travel time reliability (here measured as the difference between the $85^{\text {th }}$ and $15^{\text {th }}$ percentiles), quality of signal coordination (here measured by the percent on green), and the quality of capacity allocation (measured by the percent of cycles with split failures) are used as the basis performance measures. Next, condition thresholds are proposed to serve as the basis for these in establishing condition ratings, varying with road categories. For example, the proposed thresholds for the median travel time are shown in Table 2.4 below.

Table 2.4 Proposed condition thresholds for median travel time

\begin{tabular}{|c|c|c|c|c|c|c|}
\hline \multirow{2}{*}{$\begin{array}{c}\text { Road } \\
\text { Category }\end{array}$} & \multicolumn{2}{|c|}{ Level 1 } & \multicolumn{2}{c|}{ Level 2 } & \multicolumn{2}{c|}{ Level 3 } \\
\cline { 2 - 7 } & $\begin{array}{c}\text { Minutes/Mile } \\
\text { (maximum) }\end{array}$ & $\begin{array}{c}\text { MPH } \\
\text { (minimum) }\end{array}$ & $\begin{array}{c}\text { Minutes/Mile } \\
\text { (maximum) }\end{array}$ & $\begin{array}{c}\text { MPH } \\
\text { (minimum) }\end{array}$ & $\begin{array}{c}\text { Minutes/Mile } \\
\text { (maximum) }\end{array}$ & $\begin{array}{c}\text { MPH } \\
\text { (minimum) }\end{array}$ \\
\hline I & 1.20 & 50 & 1.50 & 40 & 31.71 & $<40$ \\
\hline II & 1.50 & 40 & 2.00 & 30 & $>2.40$ & $<30$ \\
\hline III & 1.71 & 35 & 2.40 & 25 & $>4.00$ & $<25$ \\
\hline IV & 2.00 & 30 & 3.00 & 20 & $>4.00$ & $<20$ \\
\hline
\end{tabular}

At the time of writing, such data was not available for an entire arterial network for analysis, so conceptual examples are given, with some discussion of characteristics of arterial management that may require special consideration. For example, the time-dependent properties of traffic performance fluctuate over several different periods, so a resulting system condition diagram would probably take on characteristics such as shown in Figure 2.20a. The pattern shown here indicates five weekdays followed by two weekend days, as is characteristic of traffic fluctuations. In this particular example, the weekends have relatively poor operation, indicating some effort should be spent on signal timing plans that operate on weekends.

Once data is narrowed down to the appropriate time of day, days of week, and filtered for holidays and exceptional incidents, one can begin to deduce the impacts of treatments to the operation. Figure 2.20b shows a chart of system condition over the previous 30 days, with the percentage of the system in good condition rising around day 15. This conceptual example shows how the impact of an investment like signal retiming could be visualized. This simple graphical approach would provide a much needed tool for agencies to present information about current operations and outcome assessments to decision makers and to the public. 


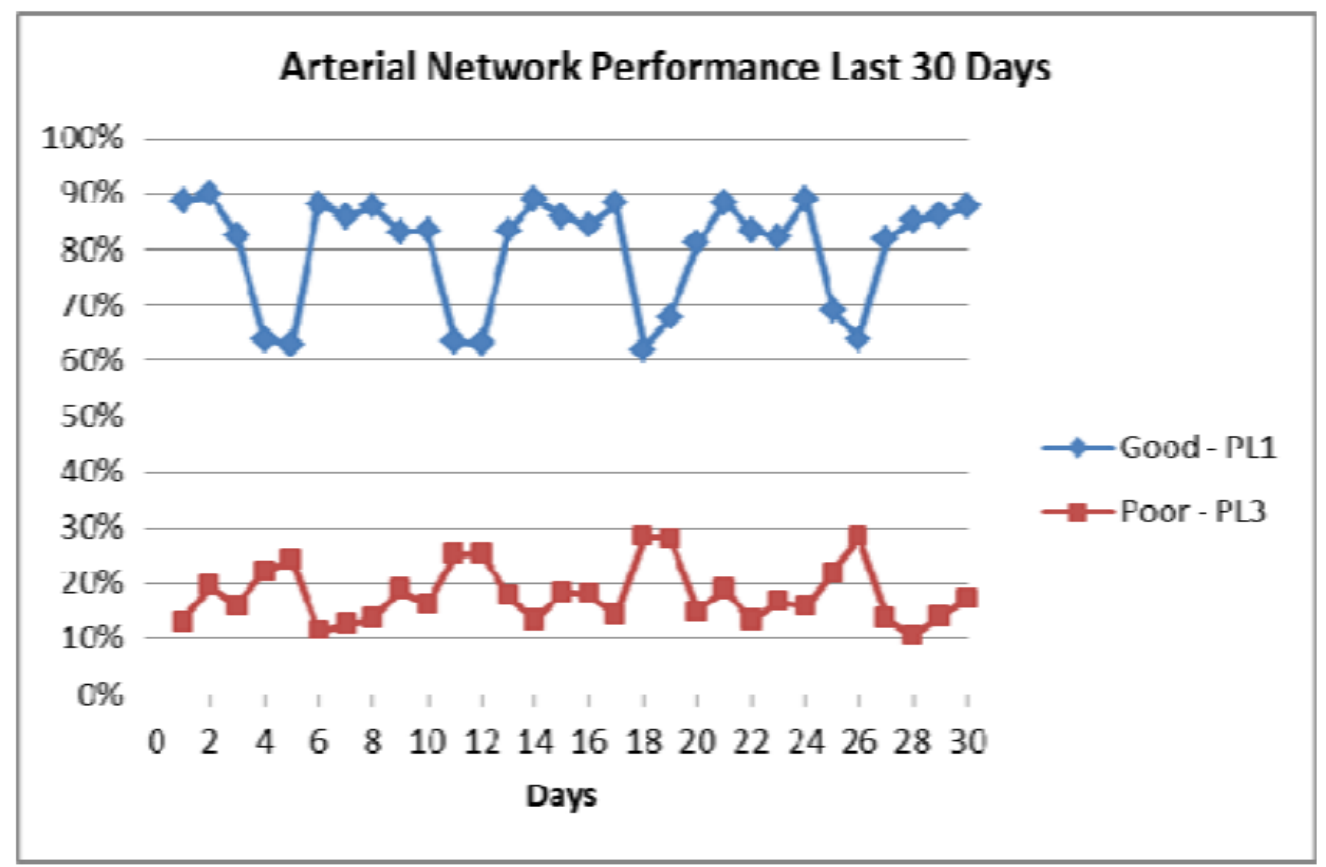

(a) A chart showing characteristic differences in weekday and weekend operations.

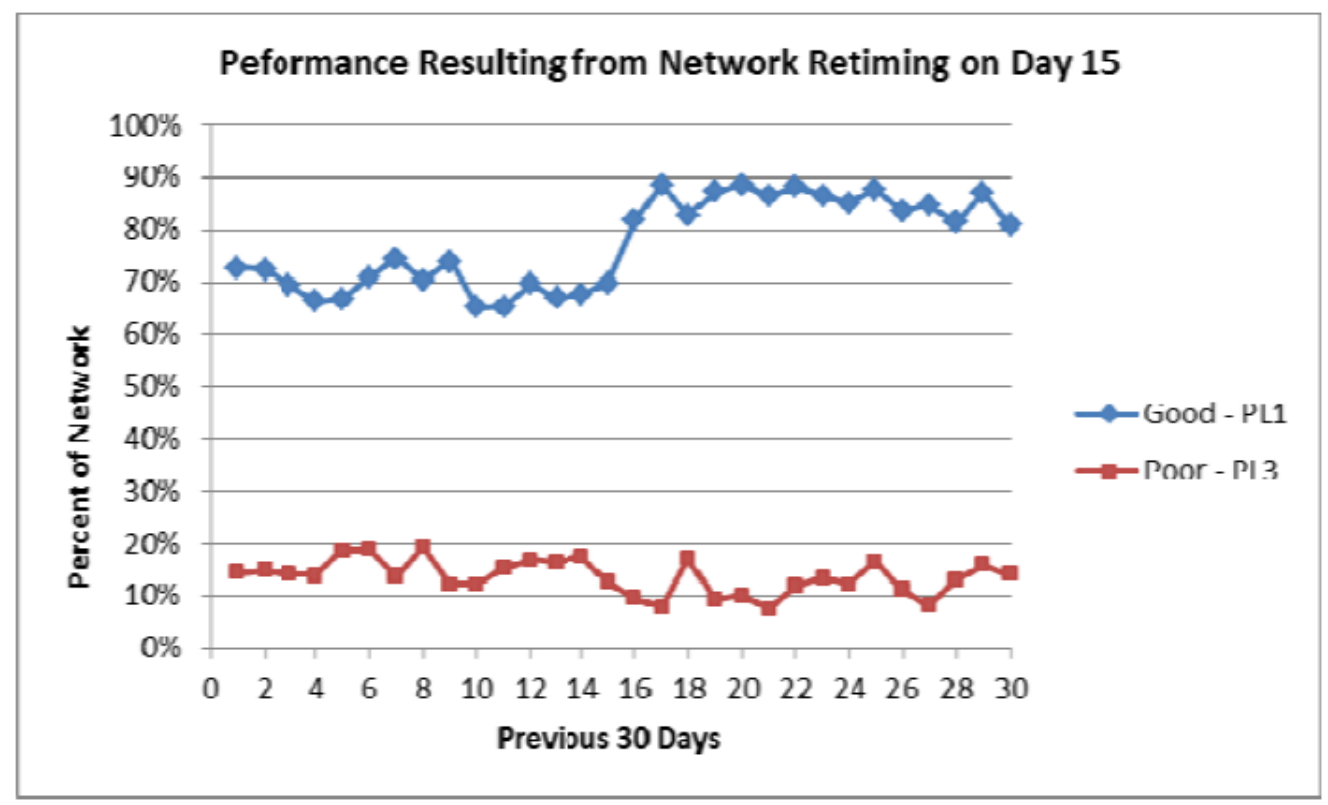

(b) A chart showing before/after impact of signal retiming.

Figure 2.20 Visualizations of arterial network performance based on percent of network operating in good and poor condition over a 30-day period 


\subsection{Graphics Report}

This report focuses on visualizations of the performance measures. It is intended as a companion document to the four-module report Performance Measures of Interrupted-Flow Roadways using Reidentification and Signal Controller Data. Rather than providing the more in-depth examination of each performance measure, the graphics report focuses on visualizations, explaining the construction and interpretation of several graphics based on vehicle re-identification data and high-resolution data.

Figure 2.21 provides a summary of the graphical performance measures highlighted in this report.

- The travel time overlay plot (Figure 2.21a) is a scatterplot where observed travel times are plotted by time of day. The observations are gathered over several days or longer to provide enough density of data to allow visualization, since one individual day may have sparser data. In Figure 2.21a, the signal plan changes are indicated by red vertical lines, which also correspond to changes in the travel time data.

- The travel time CFD (Figure 2.21b) provides a summary of travel time data for a particular data segment, such as a particular time of day. This may be gathered for one particular day or several days combined together.

- The Purdue Coordination Diagram (PCD) is a chart that shows the vehicle arrival characteristics for an individual signalized approach, typically over a 24-hour period (Figure 2.21c). The dots show individual vehicle arrivals measured with a detector, which are overlaid on the red and green intervals of individual cycles through the time period. Dots in the shaded region above the green line are arrivals in green, while those below the green line are arrivals in red.

- The percent on green (POG) is a summary metric based on the arrival data (Figure 2.21d). It is helpful for examining conditions before and after a change is made. In Figure 2.21d, the POG for four approaches at two intersections are shown; the pie charts indicate changes in the value after a change to the signal timing. A brief visual inspection finds that POG increased at two of the approaches and decreased at the other two.

- The number of split failures can be examined by looking at the red occupancy ratio (ROR) and green occupancy ratio (GOR). When both are high, it is likely that a split failure occurred. Tabulation of split failures across all the movements at an intersection enables hot spots of congestion to be identified. Figure 2.21e shows a plot of the number of split failures by movement at several dozen intersections; a handful of problem movements are easily identified by spikes in the chart.

- The individual plots of ROR versus GOR for an individual movement can also be shown, as in Figure 2.21f. The individual values for each cycle are indicated here. It would be possible for an analyst examining Figure 2.21e to "drill down" to the details of an individual movement for more information. 




(a) Travel Time Overlay Plot.

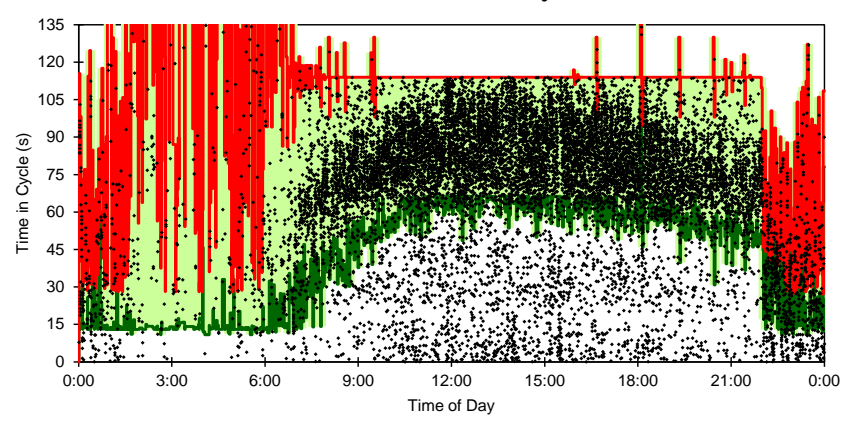

(c) Purdue Coordination Diagram (PCD).

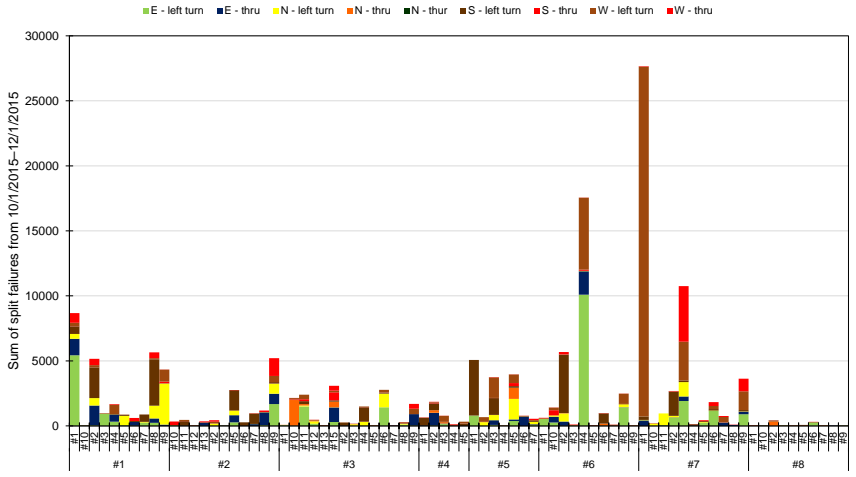

(e) Longitudinal plot of split failures.

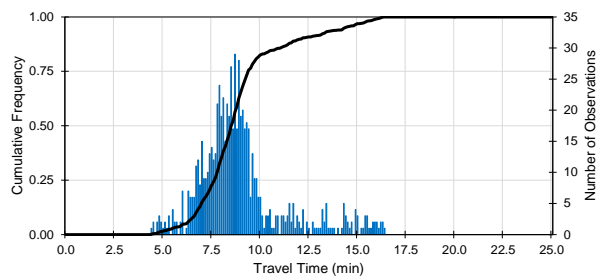

(b) Travel Time CFD.

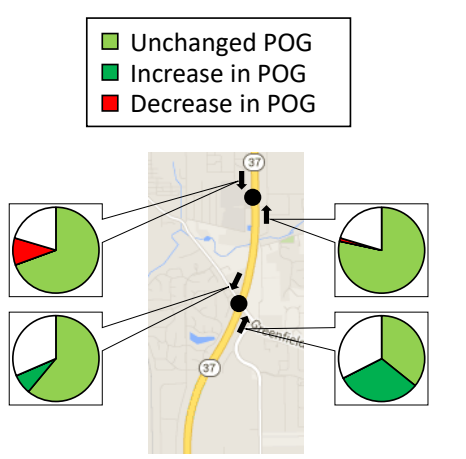

(d) Changes in Percent on Green (POG).

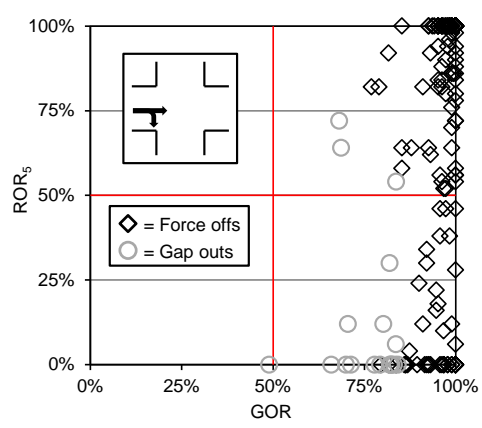

(f) ROR vs. GOR for One Phase.

Figure 2.21 Summary of visualizations discussed in the Graphics Report 


\subsection{Case Studies}

Two case studies using combined sensor data for field analysis were developed as part of this project. The first case study used data that had been collected over a 5-year period (2010-2015) on a high-speed arterial in the Indianapolis area, where the traffic signal offsets were repeatedly optimized based on traffic patterns observed in high-resolution data, and results were quantified using vehicle re-identification data. The second case study demonstrated the application of these two data sources in an unplanned detour situation where an Interstate highway was diverted onto parallel arterial highways that included a signalized section.

\subsubsection{Impact of Five Years of Offset Optimization}

This study focuses on the performance of Indiana State Road 37, which is a 10-intersection arterial highway located north of Indianapolis, Indiana. Figure 2.22 presents a map showing the location of this route. This roadway was the focus of several pilot studies on the use of high-resolution controller event data, which included the first few studies on offset optimization based on that type of data, initially with a four-intersection subset of the system in 2009, followed up with a corridor-wide study in 2010. In subsequent years, the offset optimization was repeated in 2013 and again in 2015. Each time this was done, vehicle re-identification data was collected at the same time, measuring conditions before and after the new timing plans were programmed. Thus, there were 5 years of data that could be analyzed to understand the impact of offset optimization on the traveling public. Weekday and Saturday timing plans were analyzed separately using the same method.

Comparing traffic volumes (collected with high-resolution data) with the number of re-identified vehicles found that about $5 \%$ of the total volume was captured by Bluetooth MAC address matching. The travel time distributions before and after each individual retiming were first filtered for outliers, then characterized by finding their averages and standard deviations, for comparison purposes. User benefits were calculated by scaling the travel time values using the volume and travel time value factors. For this study, travel time values were taken from the 2011 Urban Mobility Report and converted into 2015 values using Consumer Price Index differences, and a unit change in travel time standard deviation was considered equivalent to a unit change in the average travel time. Finally, the values were annualized by scaling with an appropriate conversion factor from day to year.

Figure 2.23 shows the overall results for the 5-year comparison. Each stacked bar shows the user costs in terms of travel time (TT) and travel time reliability (TTR), with the percent change in the total value for each year. Figure 2.23a shows the differences for Saturdays and Figure 2.23b shows weekdays. Each iteration was associated with a modest reduction ranging between 2.5-8.5\%. The total user benefit associated with these differences is shown in Table 2.5. Some disbenefits are observed, mostly due to directional tradeoffs within each year. That is, improvement in one direction sometime came at the cost of worsened performance in the other direction. However, the overall outcome for each pass is a net positive. The total user benefit achieved over the 5-year period is approximately $\$ 4.2$ million. 


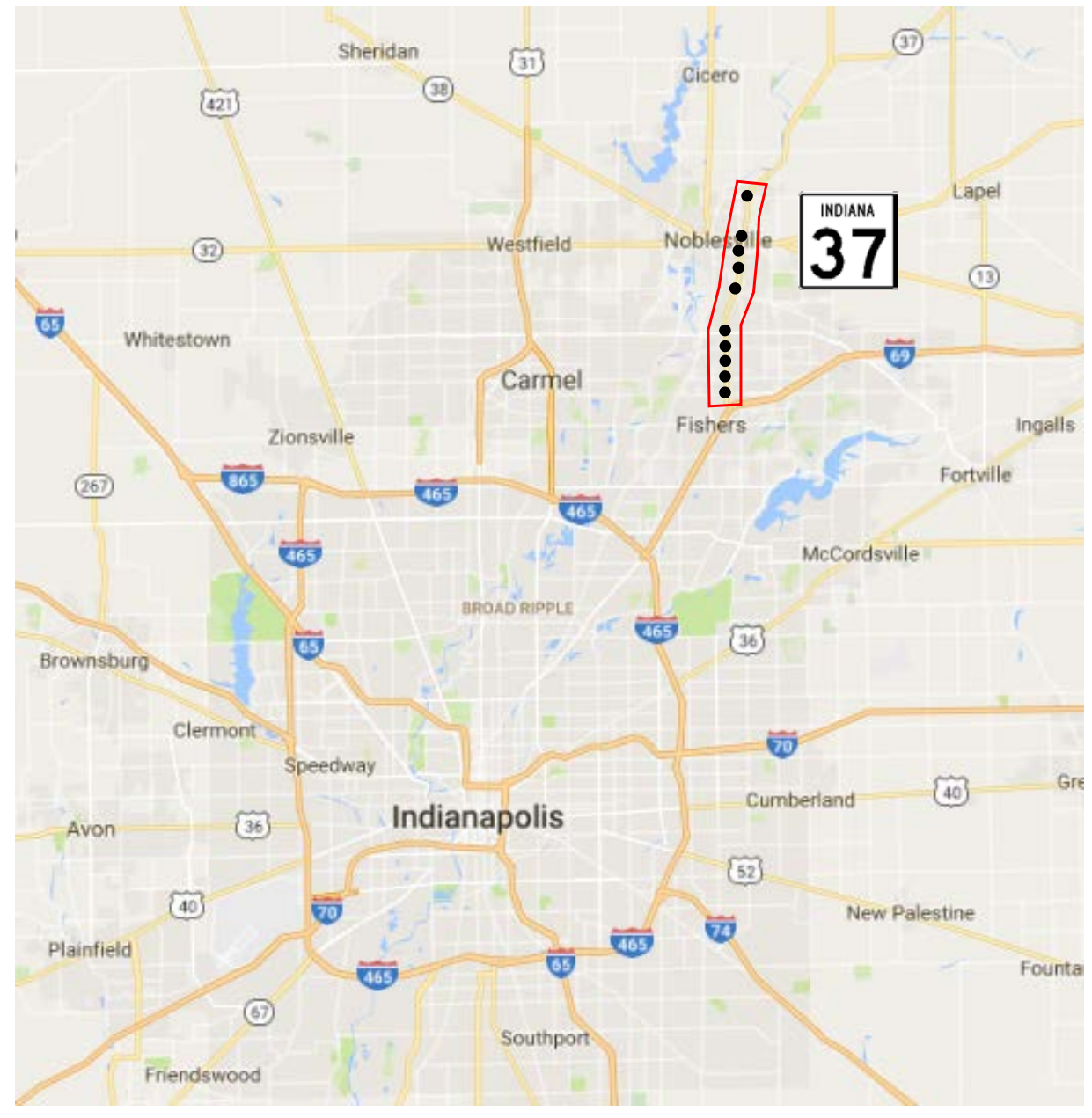

Figure 2.22 Location of Indiana State Road 37 relative to Indianapolis and neighboring communities

(a)

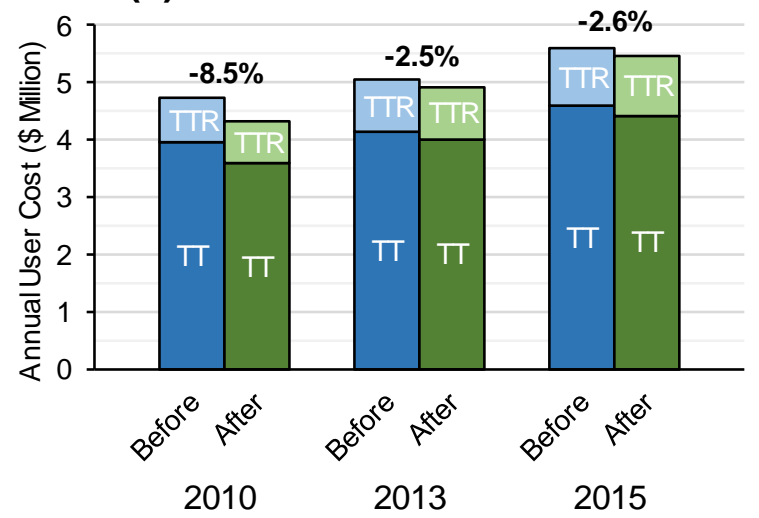

(b)

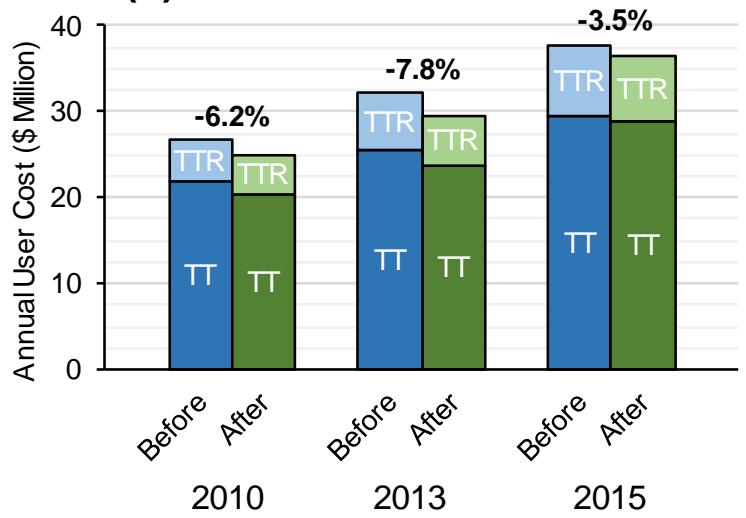

Figure 2.23 Annual travel time (TT) and travel time reliability (TTR) costs for Saturdays and weekdays06001900, before and after offset optimization. (a) Combined annual northbound/southbound TT and TTR costs, Saturdays; (b) Combined annual northbound/southbound TT and TTR costs, weekdays; 
Table 2.5 Annual TT and TTR user benefits from offset optimization.

\begin{tabular}{|l|r|r|r|r|}
\hline Comparison Group & Year & Travel Time & Travel Time Reliability & Total \\
\hline Saturday -Northbound & 2010 & $\$ 138,231$ & $-\$ 18,773$ & $\$ 119,458$ \\
\hline Saturday -Southbound & 2010 & $\$ 235,527$ & $\$ 49,557$ & $\$ 285,084$ \\
\hline Saturday -Northbound & 2013 & $\$ 307$ & $-\$ 13,732$ & $-\$ 13,425$ \\
\hline Saturday -Southbound & 2013 & $\$ 107,385$ & $\$ 32,003$ & $\$ 139,388$ \\
\hline Saturday -Northbound & 2015 & $\$ 53,942$ & $\$ 22,278$ & $\$ 76,220$ \\
\hline Saturday -Southbound & 2015 & $\$ 89,432$ & $-\$ 21,002$ & $\$ 68,430$ \\
\hline Weekday -Northbound & 2010 & $\$ 984,006$ & $\$ 498,710$ & $\$ 1,482,716$ \\
\hline Weekday -Southbound & 2010 & $-\$ 471,029$ & $-\$ 477,777$ & $-\$ 948,806$ \\
\hline Weekday -Northbound & 2013 & $\$ 961,588$ & $\$ 277,381$ & $\$ 1,238,969$ \\
\hline Weekday -Southbound & 2013 & $\$ 684,400$ & $\$ 588,986$ & $\$ 1,273,386$ \\
\hline Weekday -Northbound & 2015 & $-\$ 799,909$ & $-\$ 10,853$ & $-\$ 810,762$ \\
\hline Weekday -Southbound & 2015 & $\$ 637,189$ & $\$ 639,879$ & $\$ 1,277,068$ \\
\hline Total & & $\mathbf{\$ 2 , 6 2 1 , 0 6 9}$ & $\mathbf{\$ 1 , 5 6 6 , 6 5 7}$ & $\mathbf{\$ 4 , 1 8 7 , 7 2 6}$ \\
\hline
\end{tabular}

\subsubsection{Impact of an Unplanned Interstate Detour}

In August 2015, the northbound direction of I-65 near Lafayette, Indiana was shut down in response to unexpected settlement of a bridge pier that made it unsafe for traffic to continue using the bridge. The Indiana Department of Transportation diverted the traffic onto a 62-mile detour route, illustrated in Figure 2.24. Because of road work taking place in the city of Lafayette, it was not possible to route the detour through local roads. Also, the geometry of the ramp at exit 141 made it a better candidate to begin the detour than the alternate at exit 158.

The detour route followed northbound US 52, a four-lane divided highway, before making a left onto Indiana 28, a two-lane highway. The route then made a right turn onto US 231, which is also a two-lane highway, becoming a four-lane divided highway with traffic signals as it passes around the Lafayette area. That route included a left turn following the routing of US 231, before making one last right turn onto another two-lane section before rejoining I-65 at exit 193. I-65 was closed for approximately 6 weeks during bridge repairs.

Bluetooth devices were deployed in the field about a week after the detour began. Ten devices were deployed in total, as shown in Figure 2.24. The devices were left in the field for nine days. In addition, high-resolution data was available from most of the intersections on US 231. The high resolution data could be used to compare volumes before and after the diversion, as shown in Figure 2.25. Here, a week of volumes at the same location is presented for one week prior to the detour versus one week during the detour. Note that volumes more than doubled for much of the day, and nearly tripled during the weekends.

The Bluetooth data could be used to determine vehicle routing through the detour, as well as travel times on each alternative route. As Figure 2.24 shows, in addition to the official detour, some alternative routes are also possible. One of these follows US 52 (known locally as Sagamore Parkway) up to exit 175. This path goes through road work in Lafayette. The other leaves the detour route at Indiana 43 (known locally as River Road) in West Lafayette, and follows it up to exit 178. Figure 2.26 shows the resulting travel times and proportions of identified matches for each of the three alternatives. The data shows that about 
$60 \%$ of the diverted traffic followed the detour, with about 27\% following the Sagamore Pkwy route and 13\% following the River Rd route. The Sagamore route had a longer median travel time, and a much higher interquartile range (IQR), meaning the travel time was highly unreliable-not unexpected, due to the road work going on at the time. Motorists who chose the River Rd path did end up saving about three minutes' travel time. However, overall results were quite similar, suggesting that user equilibrium was at least approximately established in the detour area.

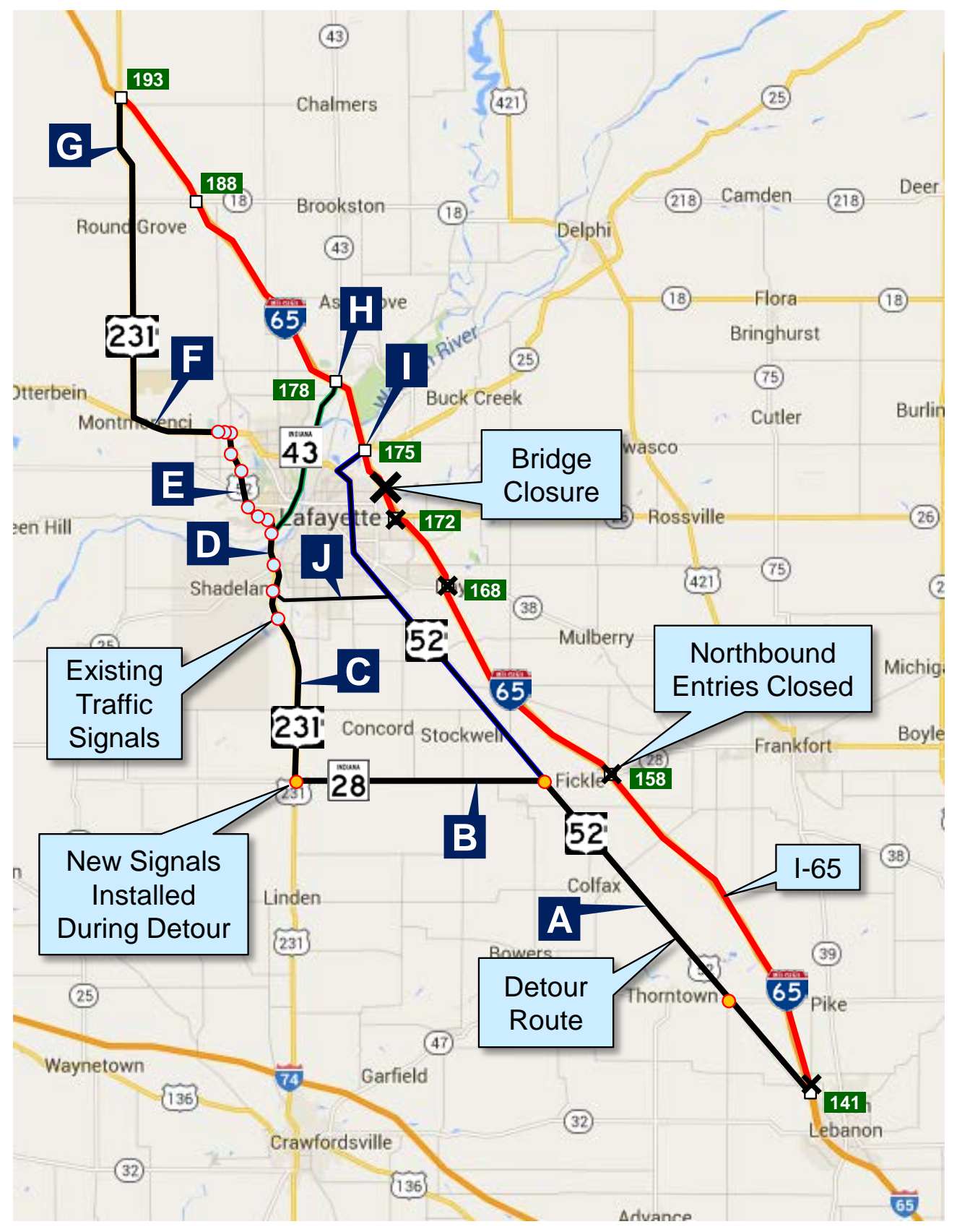

Figure 2.24 Map of the I-65 detour. The black line shows the official detour, while the blue and green lines show alternative routes. Letters A-I indicate where Bluetooth monitoring stations were located 


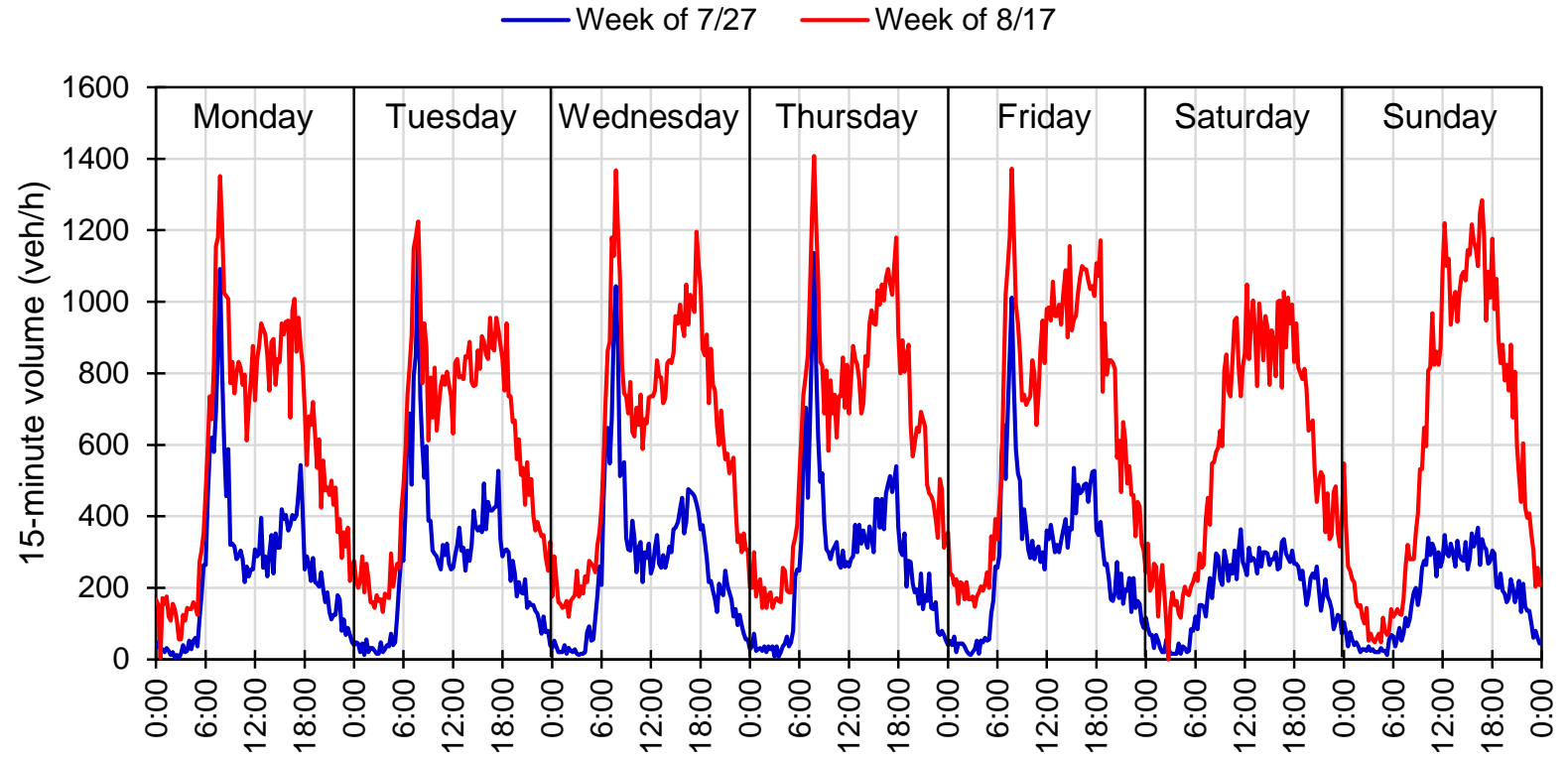

Figure 2.25 Weekly comparison of traffic volumes before and after the I-65 detour 


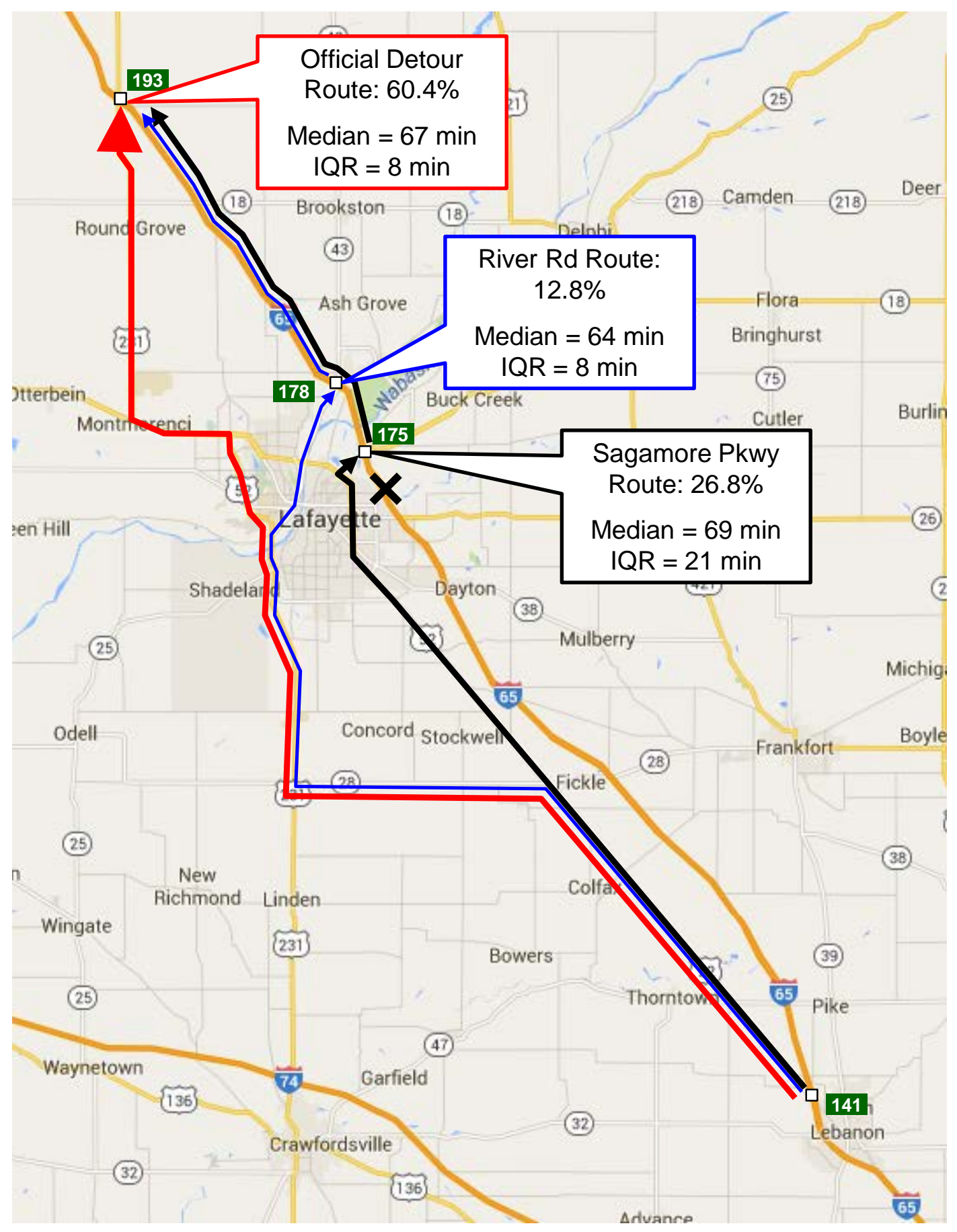

Figure 2.26 Map of origin-destination routes taken and travel times experienced on each 
Figure 2.27 shows results for detour route travel times. Figure 2.27a shows the travel times from the origin point to several destinations, while Figure 2.27b shows the amount of delay occurring on individual segments along the route. Figure 2.27a shows the slope of the curve gradually becoming more horizontal with distance, which is a fairly typical pattern for arterials as delay accumulates at intersections. Figure 2.27b shows that the highest amount of delay is actually seen on the first and second segments, which include situations where two lanes of traffic merge into a single lane and make a left turn (segment A to $\mathrm{B}$ ), or where that single lane of traffic makes a right turn (segment B to C). The remaining segments have some delay but it is lower in magnitude.

The high-resolution data was also used to evaluate the efficacy of a signal timing plan that was implemented for the detour. Figure 2.28 shows a more detailed map of the signalized portion of US 231, which includes 12 intersections. At the time that the detour began, only intersections 4-8 operated as a coordinated system along US 231, which was set up for a much lower volume of traffic. Intersections 1-3 were not coordinated, intersection 8 was not yet signalized and was operating as two-way stop controlled, and intersections 10-12 were coordinated for east-west traffic. By the time that the data collection effort was underway, all twelve intersections had been coordinated under a new timing plan intended to shunt northbound traffic through the system as efficiently as possible. High-resolution data was available for eight of the twelve intersections.

Figure 2.29 shows PCDs for all of the intersections from which data was available during a representative date during the detour after the emergency plan was deployed. A visual glance at the PCDs finds evidence of a well formed platoon captured within the green band at each intersection along the route. Progression was also fairly good in opposing southbound direction, as well. Table 2.6 shows the results in terms of the percent on green (POG), as well as the green-to-cycle ratio $(g / C)$ and the platoon ratio $\left(R_{p}\right)$ for each approach in the system for which there was data. Northbound POG values were found to exceed $80 \%$ for all of the approaches except for the entry approach (Int. 1) in the northbound direction, and several had POG above $90 \%$. Platoon ratios exceeded 1.1 for most, further indicating better progression than under random arrivals (as indicated by $R_{p}=1.0$ ). Travel in the southbound direction was not considered when designing the timing plan, but the results show that it performed reasonably well at most approaches. The good quality of progression in the northbound direction explains why there was relatively little delay associated with the sections of the signalized arterial in Figure 2.27b. Sections $\mathrm{C}$ to $\mathrm{D}, \mathrm{D}$ to $\mathrm{E}$, and $\mathrm{E}$ to $\mathrm{F}$ have much less delay than A to B and B to C. Section E to F has the highest amount of delay among the signalized arterial sections, likely because it includes a left turn at Int. 10, as shown in Figure 2.28.

Final Report

Contract \#: DTFH61-14-C-00035 


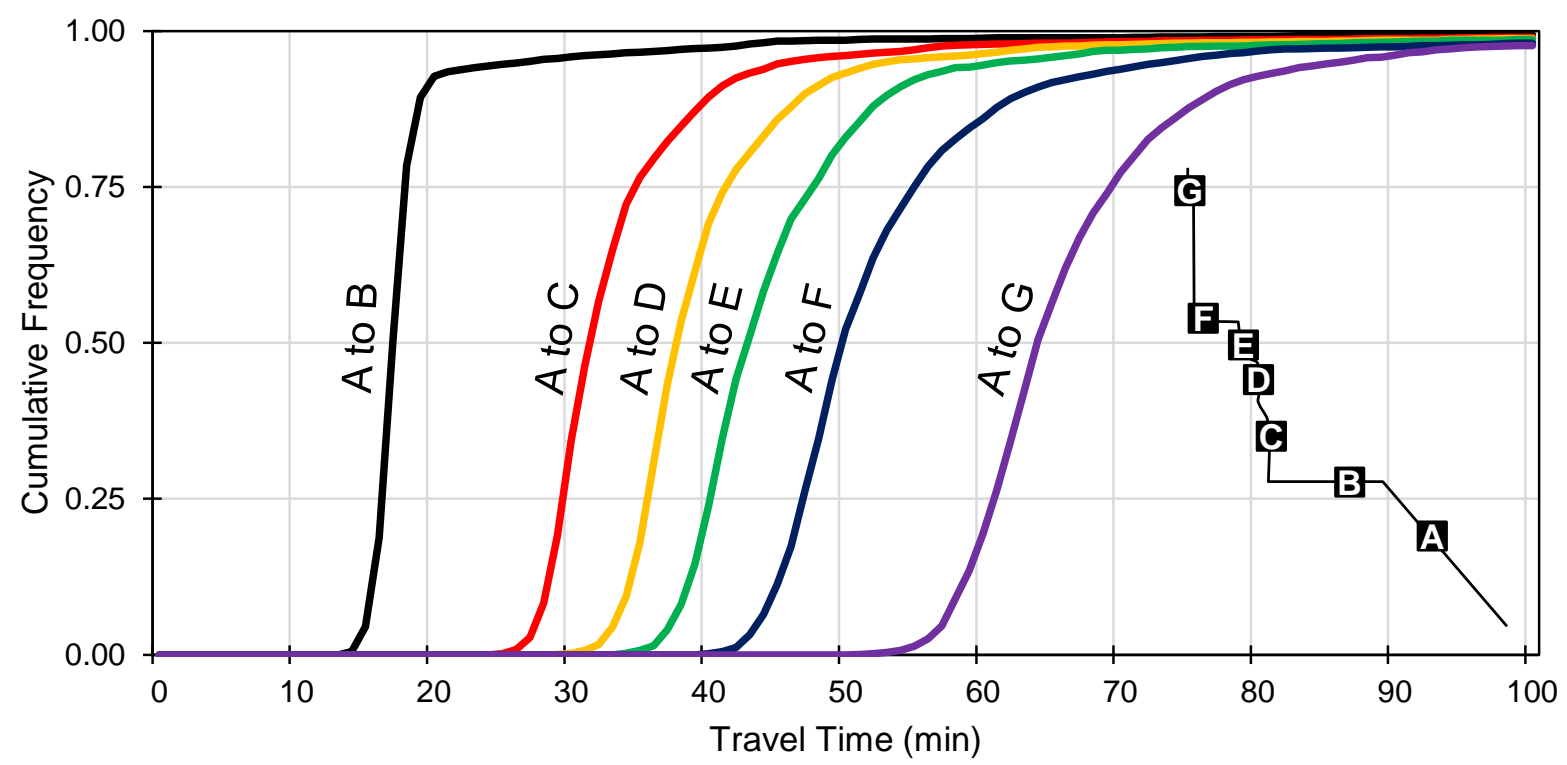

(a) Travel time from origin to destination

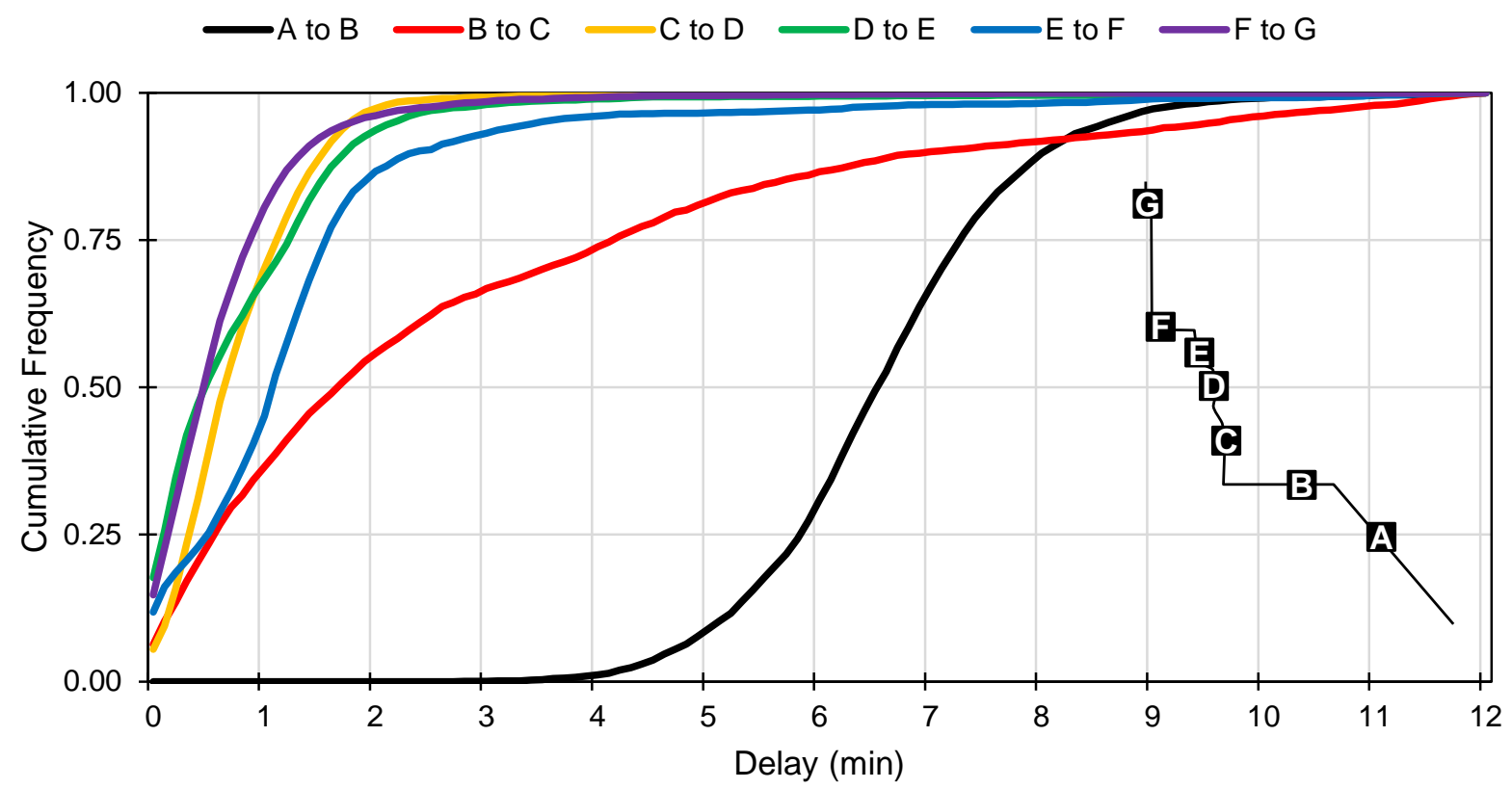

(b) Northbound delay by segment

Figure 2.27 Travel time data during the I-65 detour 


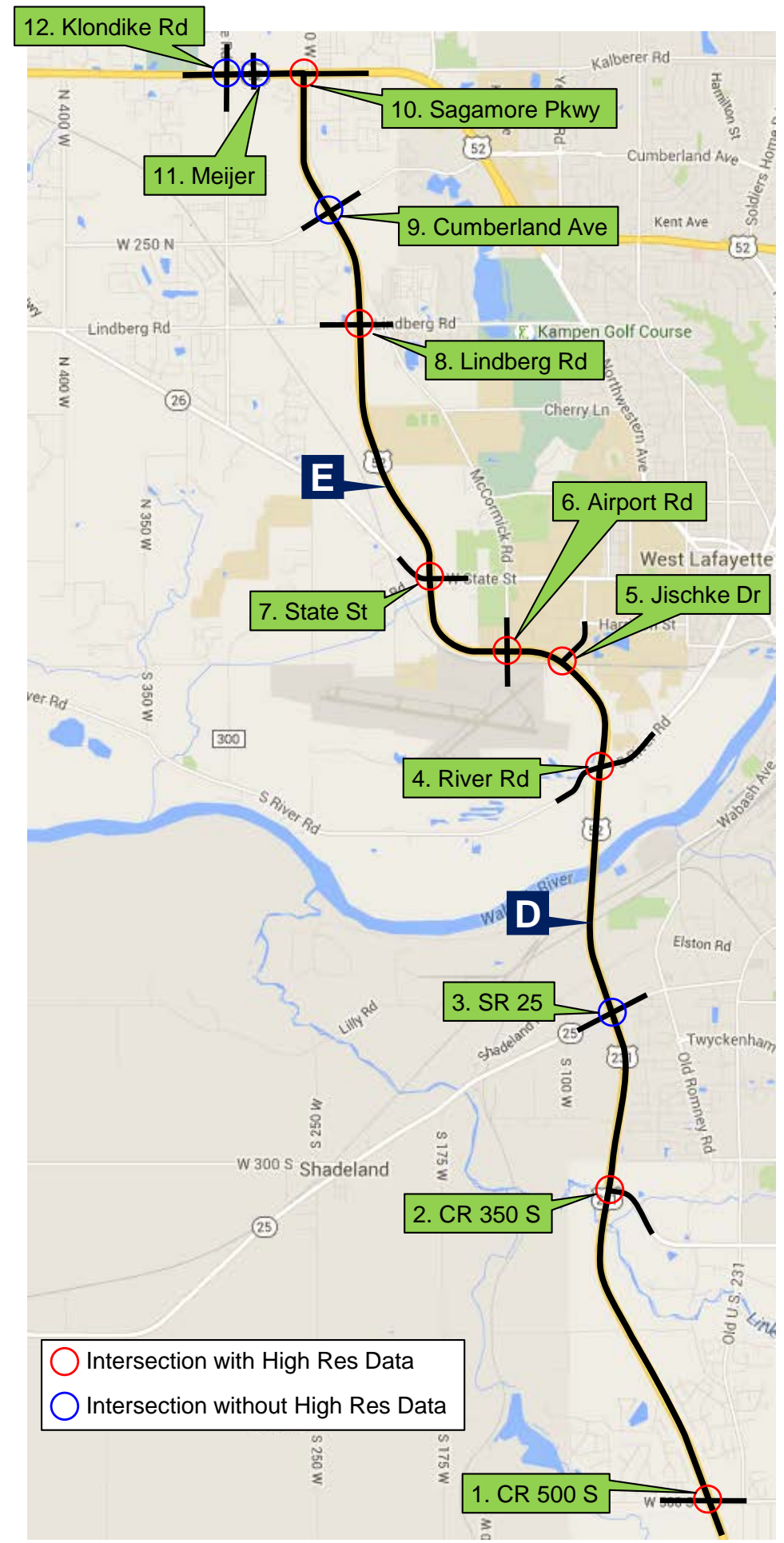

Figure 2.28 Map of the existing signalized portion of US 231 

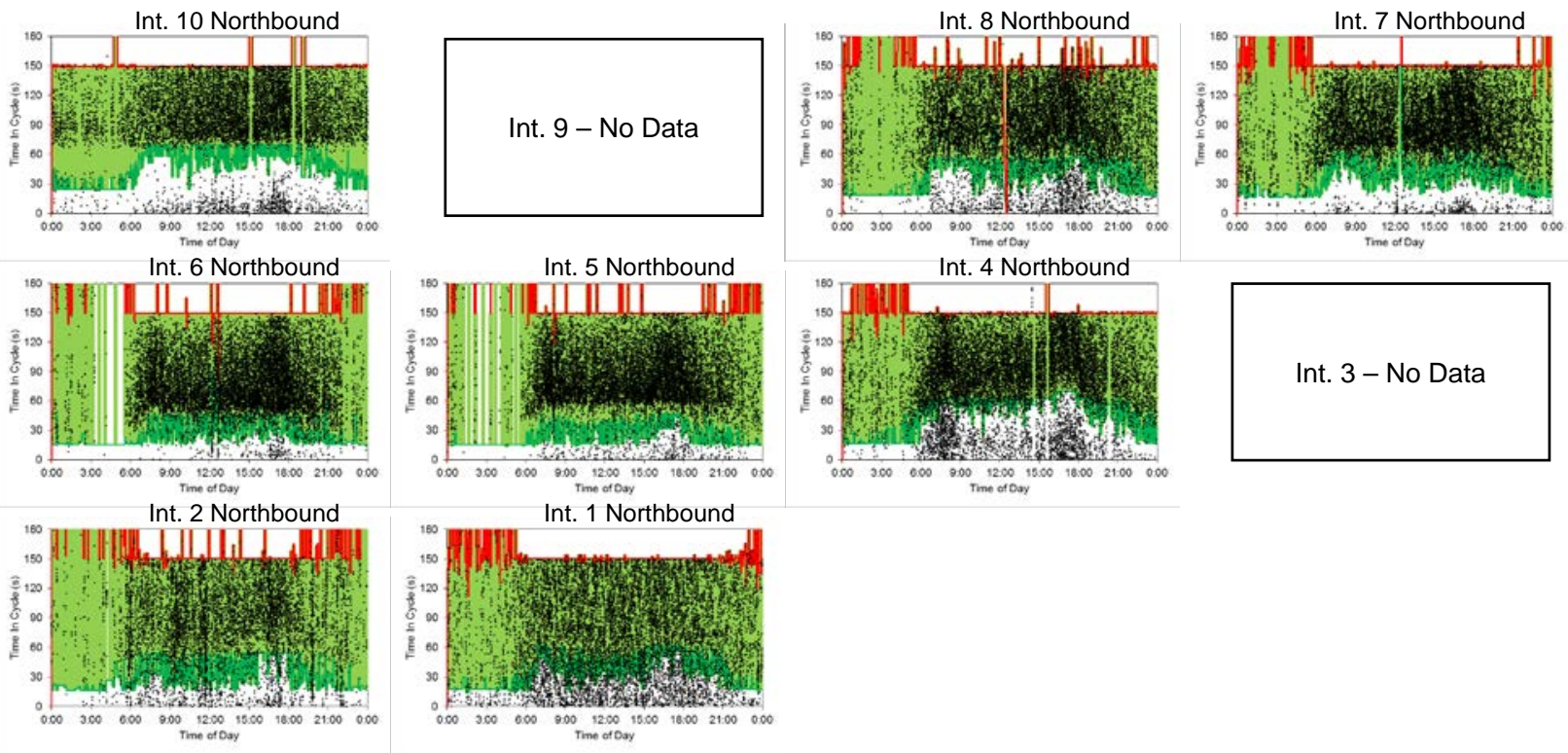

(a) Northbound
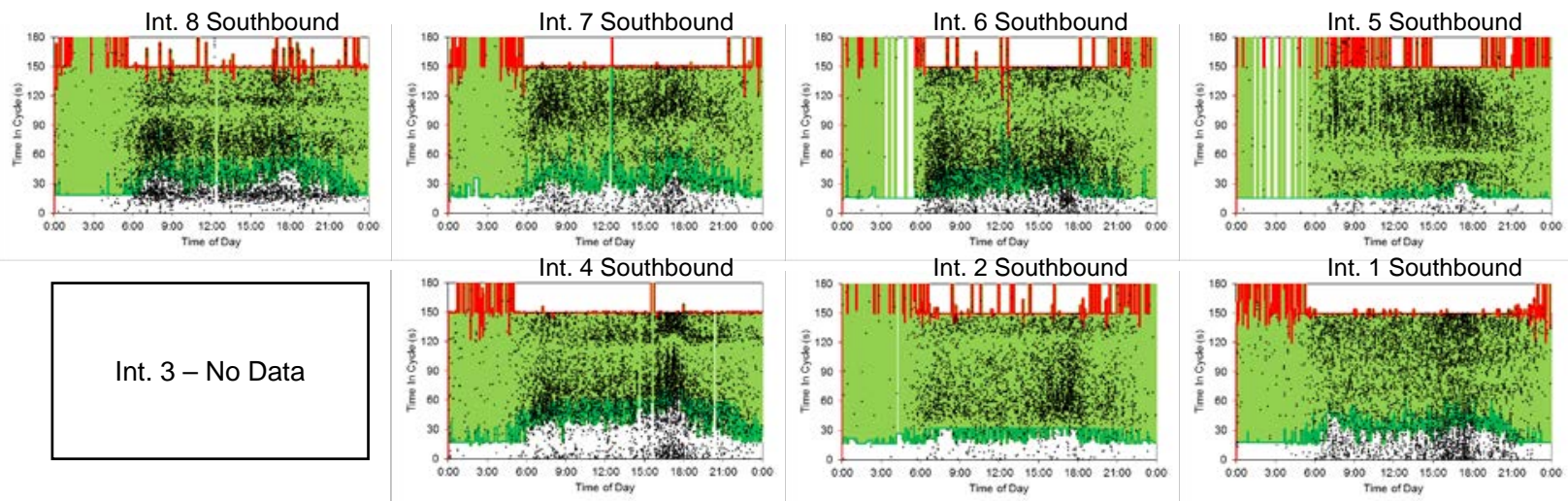

(b) Southbound

Figure 2.29 PCDs from the US 231 corridor. 
Table 2.6 Summary performance metrics for progression along US 231

\begin{tabular}{|l|c|c|c|c|c|c|}
\hline & \multicolumn{3}{|c|}{ Southbound } & \multicolumn{3}{c|}{ Northbound } \\
\hline Intersection & POG & $g / C$ & $R_{p}$ & POG & $g / C$ & $R_{p}$ \\
\hline 10 & \multicolumn{3}{|c|}{ N/A } & $90.2 \%$ & $66.8 \%$ & 1.35 \\
\hline 9 & \multicolumn{3}{|c|}{ No Data } & \multicolumn{3}{c|}{ No Data } \\
\hline 8 & $70.3 \%$ & $77.4 \%$ & 0.91 & $88.2 \%$ & $76.3 \%$ & 1.15 \\
\hline 7 & $82.8 \%$ & $79.7 \%$ & 1.04 & $94.5 \%$ & $77.6 \%$ & 1.22 \\
\hline 6 & $79.7 \%$ & $85.9 \%$ & 0.93 & $95.8 \%$ & $86.1 \%$ & 1.11 \\
\hline 5 & $96.4 \%$ & $91.7 \%$ & 1.05 & $96.4 \%$ & $86.4 \%$ & 1.12 \\
\hline 4 & $74.5 \%$ & $72.1 \%$ & 1.03 & $80.8 \%$ & $68.4 \%$ & 1.18 \\
\hline 3 & \multicolumn{3}{|c|}{ No Data } & & No Data \\
\hline 2 & $95.8 \%$ & $87.0 \%$ & 1.10 & $89.5 \%$ & $82.4 \%$ & 1.09 \\
\hline 1 & $75.6 \%$ & $80.5 \%$ & 0.94 & $76.0 \%$ & $77.4 \%$ & 0.98 \\
\hline
\end{tabular}




\section{Chapter 3: Resources to Support Performance Measurement}

This project includes several products to encourage widespread adoption of the breakthroughs in arterial characterization using new data sources. These resources include a lexicon of terms as well as three software development efforts that serve as reference implementations for the performance measure visualizations and data standards developed previously in this initiative. Consistency of language, data standards and reference-able pseudo-code, when used in conjunction with prevailing procurement practices, encourage uniformity of application within the industry, create increased value for the customer (typically a transportation jurisdiction), and hasten adoption of best practices.

Improvements in technology and methods are often accompanied by a confused and fragmented market as vendors vie for market share. Vendors typically provide proprietary technology and software spanning sensor field hardware all the way to analytics and visualizations from back-office processing software, resulting in wide variance in implementation from vendor to vendor and a corresponding variance in user experience. As technology proliferates and market matures, price competition begins to enforce uniformity and interoperability through procurement specifications. Product consistency across vendors, and uniformity of customer experience are signs of a mature, standards-driven market place. The reidentification market finds itself at a transition point. Initial product offerings by vendors have been vertically integrated solutions based on vendor specific (typically proprietary) software and data structures integrated with hardware (sensor) offerings. Interoperability of re-identification equipment with down-stream processing for performance measures and visualization are NOT the norm in the market. Currently data analytics options available to customers are limited to the analytics offered by the equipment vendor unless the customer invests in a significant and costly data integration process.

A goal of this project is to provide additional tools to encourage best practice and uniformity in the market. In addition to a lexicon of terms, this toolset includes the creation of a re-identification segment travel time data standard, referenced as CWS5200, implementation of the arterial performance measure and network performance metrics based on the CWS5200 in an open source software known as VPXplore, and the implementation of the RTLC in Traffax's BluSTATs analysis software along accompanying algorithm documentation. Combined, these tools will encourage market maturation creating uniform expectations and experience for the customer, increasing customer value, extending the shelf-life of data, and ultimately faster market growth.

\subsection{Lexicon}

The project's lexicon is intended to complement other project deliverables by providing background information, definitions, and clarification of terms and acronyms. It draws on concepts, language and ideas from the transportation engineering field in general. For this work, such terms, at times, take on specific or narrower meanings than that used in common vernacular.

\subsection{Common Data Format}

This SBIR initiative also targeted the delivery of common data formats to facilitate adoption of, and uniformity within, arterial performance measures from re-identification and high-resolution controller data (HRCD). The traffic data industry has typically been characterized by independent data collection activities to support separate and distinct data analysis activities. For example, data needed for long term planning of roadway capacity was collected separately from the data needed by operations personnel 
responsible for day to day management of traffic and incidents. A common data format allows for more for efficient data collection from newer and richer data sources such as re-identification and HRCD, allowing data collection activities to support numerous analysis functions such as traffic operations, transportation management centers and long-term planning. Data standards also allow for cost efficiency during procurement, insuring the output from different vendors can support data analysis activities without custom integration of vendor specific data feeds.

A common HRCD format would minimize the variability of implementation from vendor to vendor. A significant step toward a common data format for HRCD was completed by a team lead by Purdue in 2012, in a document named INDIANA TRAFFIC SIGNAL HI RESOLUTION DATA LOGGER ENUMERATIONS [14]. These enumerations, or numbered codes, have been used effectively to combine data from multiple signal control vendors in early implementations. Although not a fully formatted data message standard, such as an XML application programming interface (API) data feed, the enumerations provide common definitions, and set expectations within the industry for uniformity. Indeed they have been widely accepted, and now all major signal manufacturers support the enumerations.

Within this initiative a standard data format was developed for segment level travel time data based on reidentification technology. The standard was developed in conjunction with the University of Maryland Center for Advanced Transportation Technology (CATT) research and analytics branch, called CATTWorks. As such the standard is labeled as the CWS5200 re-identification data standard where CWS stands for CATTWorks Standard. This standard format is intended to support various forms of reidentification technologies such as Bluetooth, Wi-Fi, automated license plates readers, and toll tag readers to name a few. The CWS5200 standard is intended to provide a uniform method of conveying observed travel times collected along corridors using any form of re-identification technology, and a complete description is available in the Common Data Format Report. 


\subsection{Arterial Performance Measures Software}

This Small Business Innovative Research (SBIR) project includes aspects of software development meant to encourage widespread adoption of the breakthroughs in arterial characterization resulting from the use of new data collection methods such as re-identification data. The software developed to support arterial performance measures is intended to encourage uniformity of application by providing reference routines (or pseudo-code) for implementation of the performance measures and data standards developed in this initiative. A full description of the arterial performance measures software, including code listings and source code access is available in the project report entitled, "Arterial Performance Measures Software” [15].

The software reference routines are provided in a standard numerical processing and visual scripting language, Matlab ${ }^{\mathrm{TM}}$, common to the analysis and R\&D community. These reference software routines are embedded in an analysis tool, jointly developed between Traffax Inc. and the University of Maryland (UMD) Center for Advanced Transportation Technology (CATT), referred to as VPXplore. VPXplore, which originated as a set of Matlab ${ }^{\mathrm{TM}}$ scripts to assist in comparing traffic data from different sources, has evolved into open source software for the comparison and visualization of re-identification and commercial probe vehicle data.

Support for the CWS5200 data standard was implemented in both VPXplore and BluSTATs, Traffax's engineering analysis software for Bluetooth and WiFi re-identification data, allowing the porting of processed sensor data to common open source platforms. BluSTATs is representative of any vendor's custom analytics processing software. VPXplore was augmented for use with CWS5200 data standards, and extended for overlay and cumulative frequency diagram visualizations for arterial performance measures, all operating on CWS5200 data sets. The flowchart in Figure 3.1 is representative of the type of open data format evolution needed to unlock travel time information from proprietary vendor-specific analytics packages and to support a larger variety of public agency needs.

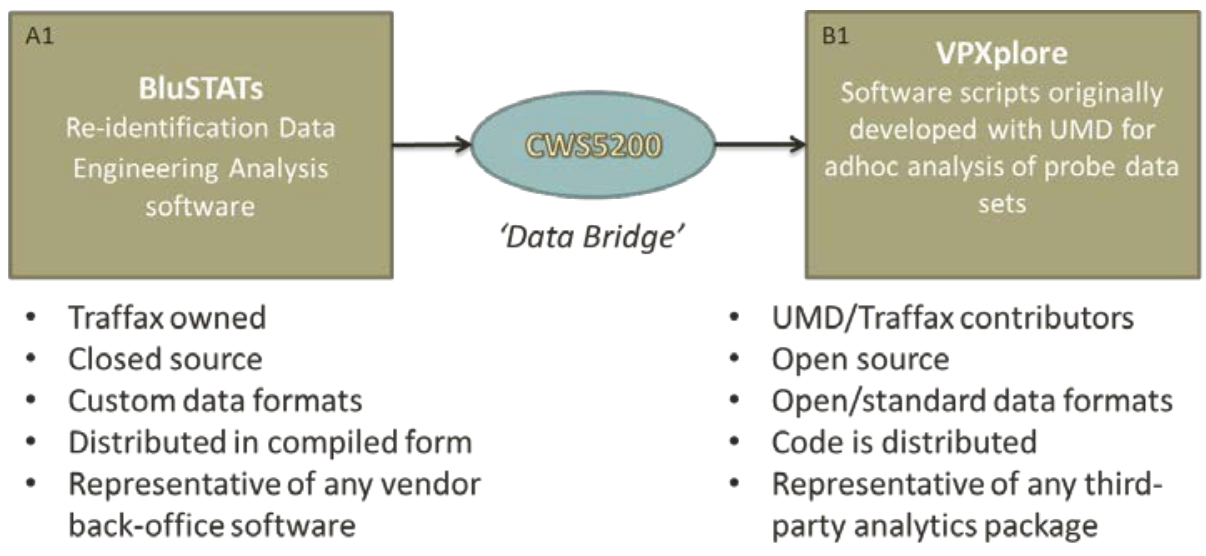

Figure 3.1 Implementation framework of the CWS5200 data standard

Representative output from VPXplore is shown in Figure 3.2 below showing a comparison between travel times produced from a commercial vehicle probe data set and a re-identification data set for the same facility. The data sets are compared using both the 24 hour overlay view and the cumulative frequency distribution (CFD) chart. 


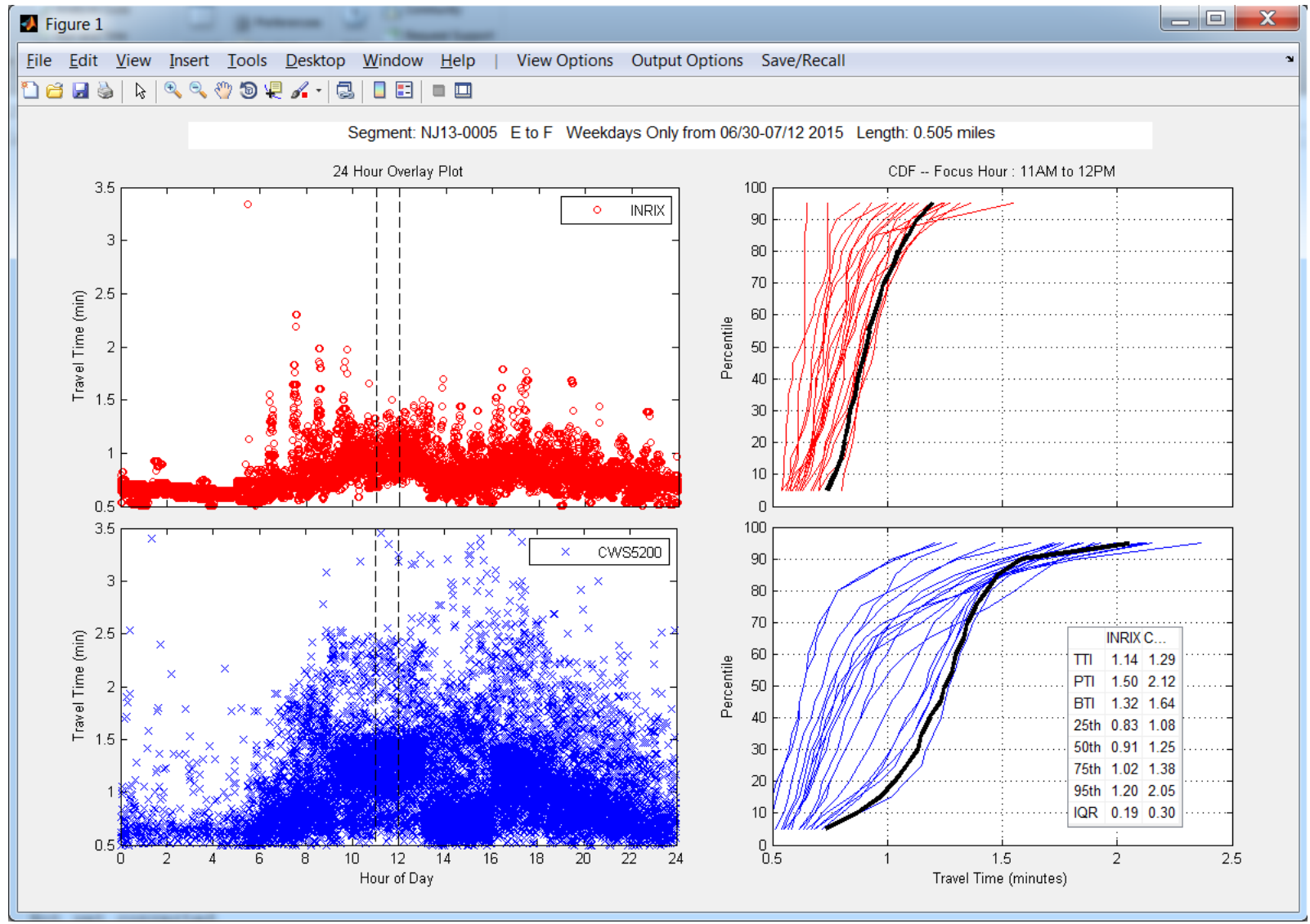

Figure 3.2 Representative output of VPXplore between two data sets using overlays and CFDs 


\subsection{Network Metrics Software}

In this initiative, the VPXplore open-source software (as described in 3.3 above) was extended to support the base travel time network performance measures developed in "Network Performance Measures for Arterials - a Systematic Level Perspective” [16] (referred to as the NPMA report), and as summarized in section 2.4 above. The NPMA report introduced a framework for arterial network performance measures and management patterned off of other infrastructure management systems and based on four key condition performance metrics from either re-identification travel time or high-resolution controller data.

VPXplore was augmented to provide the two measures emanating from re-identification travel time data sets which include: (1) median travel time measured in minutes per mile, or miles per hour, and (2) travel time reliability, implemented as the difference in the $15^{\text {th }}$ and $85^{\text {th }}$ percentile travel time, divided by the median travel time for normalization.

NPMA support is built upon the VPXplore software architecture, leveraging the integration of the CWS5200 standard data format for segment level re-identification data. The implementation of VPXplore required two additional software modules as illustrated in Figure 3.3 below. . The first module, Analytics Processing, calculates the specific performance measures called out in the NPMA report based on the CWS5200 data structure. The second module, the Visualization Engine, produces customized visualizations of the NPMA performance measures, a sample output of which is illustrated in Figure 3.4.

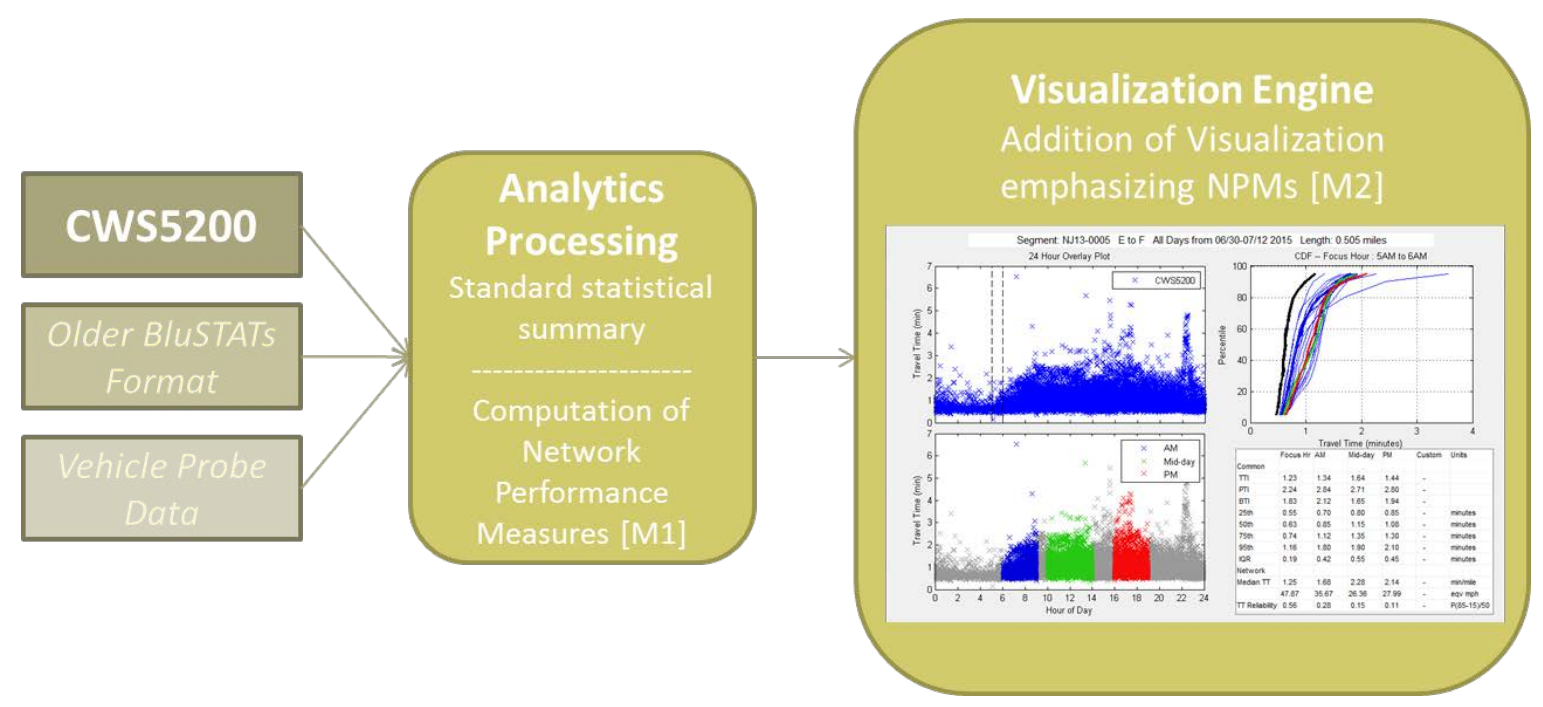

Figure 3.3 Augmentation of VPX Software for Network Performance Measures 


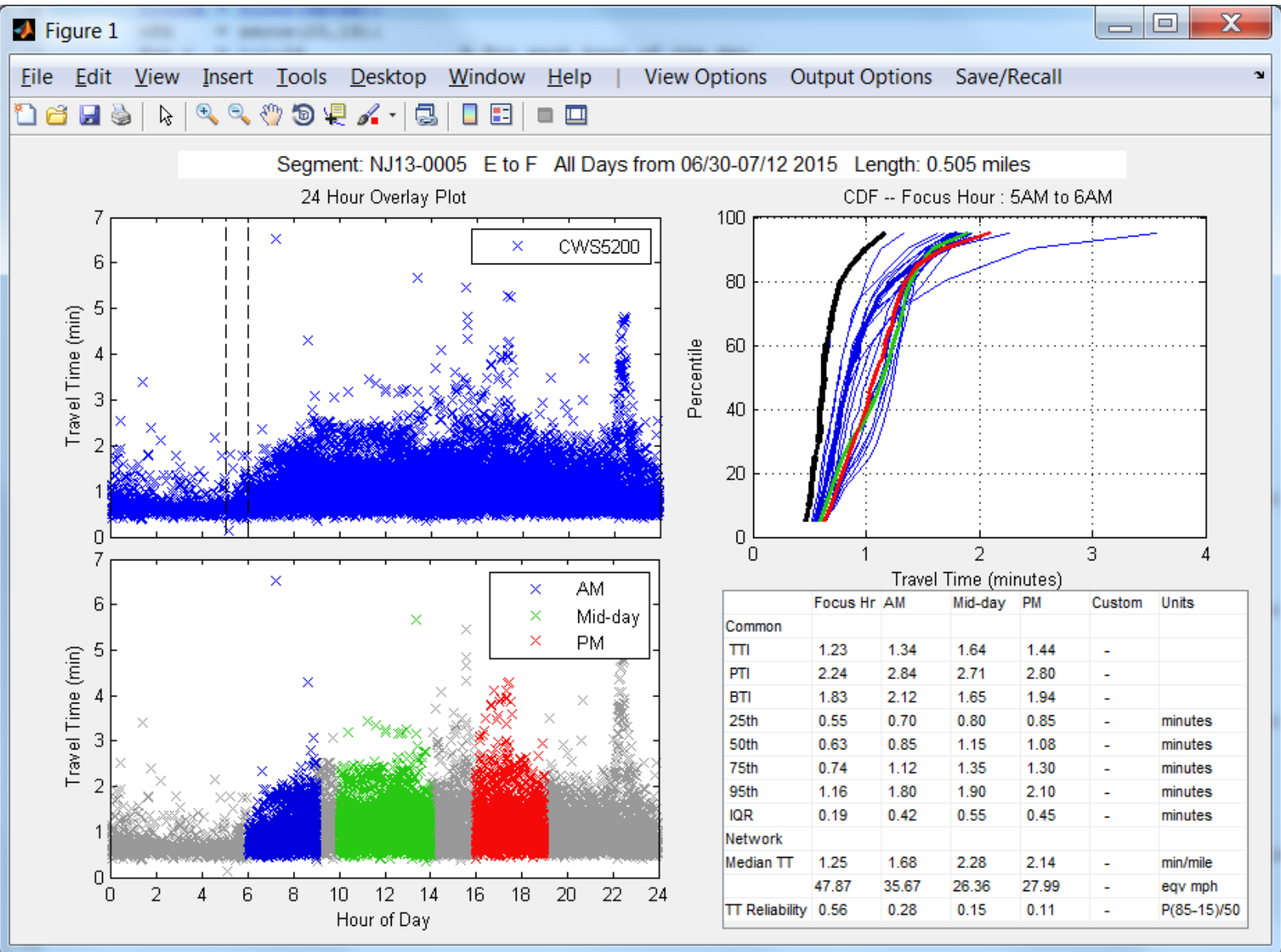

Figure 3.4 Sample output of the Network Performance Measures implemented in VPXplore

\subsection{Software Demonstration}

A recorded demonstration was prepared explaining the purpose, development, framework, and potential uses of the developed open-source software. The recorded presentation can be viewed at: https://webmeeting.umd.edu/p3c5ymxiq97/?OWASP CSRFTOKEN=e1c84f4446075128a4ceb0979ccc05c3c12da7eb212a4 f14ca957d2e11c4514c and the PowerPoint slide deck can be accessed at https://tinyurl.com/ydz7dm3j. 
The implementation of the roadway trip length characteristic (RTLC), as discussed in Section 2.3 of this report, is carried out in the BluSTATs software, owned and maintained by Traffax Inc., and distributed in a compiled form for the Windows ${ }^{\mathrm{TM}}$ operating environment. The RTLC operates on vehicle detection data from the sensors within a corridor before it is aggregated to segment level travel times. The goal of the software, along with its detailed report entitled "Arterial Trip Length Characteristics Software” [17], is to provide a reference for implementation, sort of a standard recipe, for the RTLC. The software operationalizes the RTLC statistics for use on data collected in the field, and the associated report [17] provides a detailed explanation of the processing sequence and algorithms needed for implementation.

A diagram of the approach is shown in Figure 3.5 below. Data from field sensors are input into BluSTATs. Station processing performs cleaning, filtering and aggregating to provide detection data in a station data structure format. Station detection data sets are then input into the RTLC processing which consists of two processing steps.

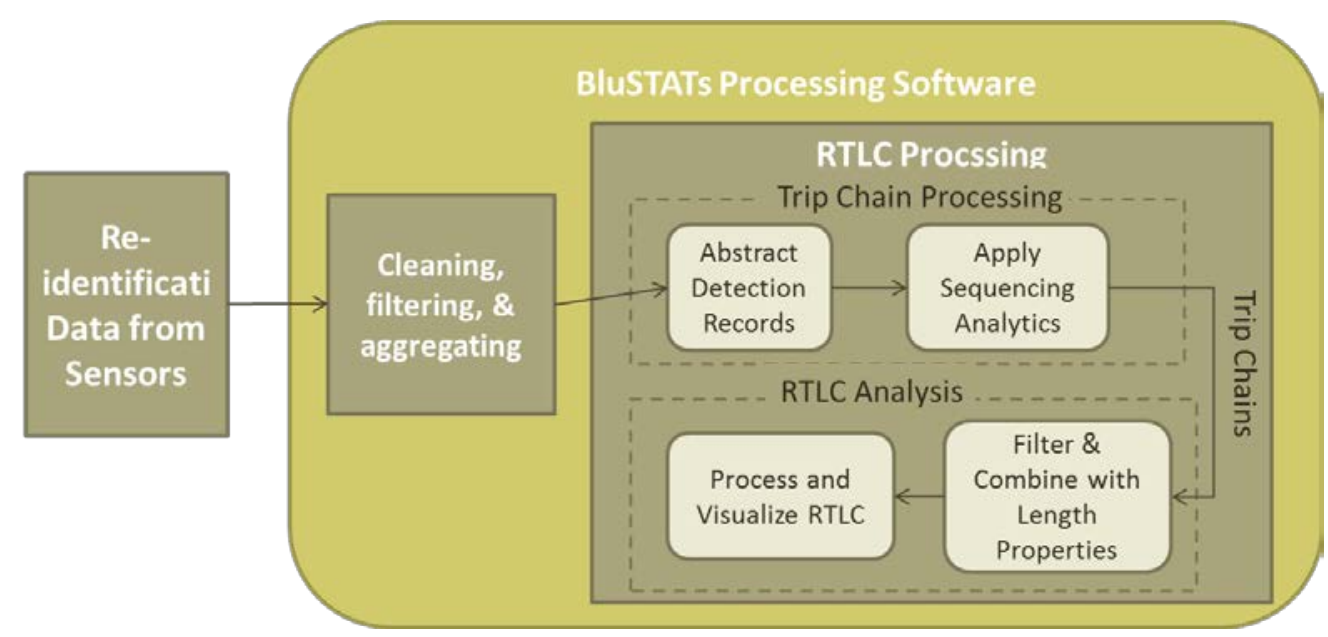

Figure 3.5 Implementation of RTLC within BluSTATs

The first process is a trip chain analysis in which the vehicles are observed as they progress through a series of stations, resulting in what are referred to as trip chains. Trip chains are denoted in a short-hand format in which each station along a corridor is assigned a letter in the order of progression along the corridor. The first station is assigned ' $A$ ', the second ' $\mathrm{B}$ ', the third ' $\mathrm{C}$ ' and so on. The short hand notation provides the path taken on the corridor. The trip chain 'BCD' indicates the vehicle was first observed at station B, then $\mathrm{C}$, and lastly $\mathrm{D}$. The analysis of trip chains results in a tabulated list of unique trips observed, and the total number of observances of that trip, a sample of which is shown in Figure 3.6 below. Trip chain analysis is a foundational step that contributes to various analytics, and contains optional filtering parameters. 


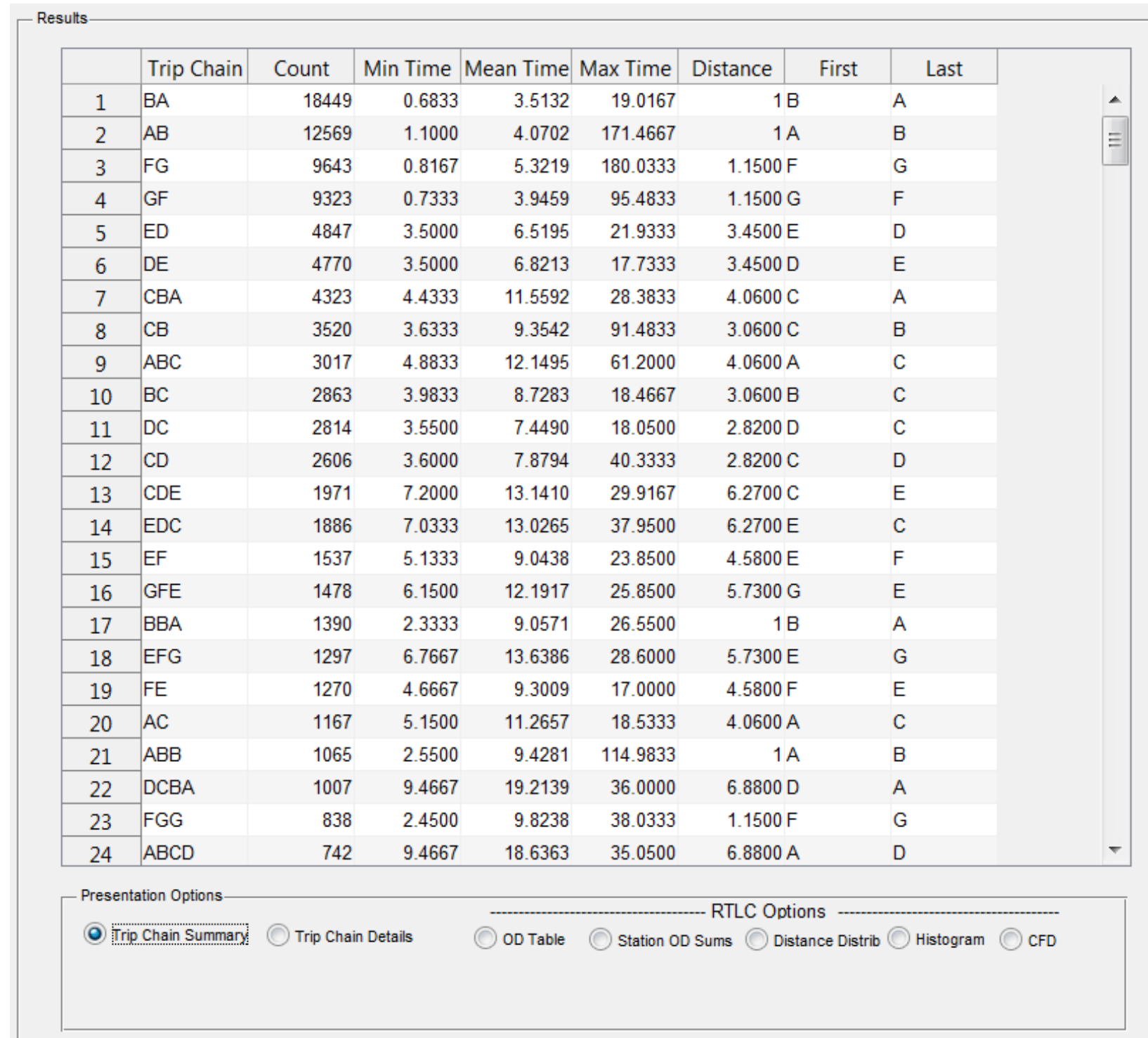

Figure 3.6 Trip chain summary table for US-1 with RTLC filter options applied

The second process creates the RTLC using the information in the trip chain summary table combined with distance information and additional filtering that removes noise and outliers, and consolidates output. These filters eliminate zero length trips, loop-back trips, and omit trip chains observed below a specified minimum observation threshold. With additional filtering and tabulated trip distances (as shown in Figure 3.6) the data in the trip chain summary table is transformed into various tables providing trip characteristics. One such table is the trip length distribution as shown in Figure 3.7, which forms the basis for histograms and cumulative frequency distributions (CFDs) of the RTLC. A normalized histogram produced from the trip length distribution table is illustrated in Figure 3.8. 


\begin{tabular}{|c|c|c|}
\hline - Results - & & \\
\hline & Distance Bin & Count \\
\hline 1 & 0 & 56473 \\
\hline 2 & 2.3000 & 36598 \\
\hline 3 & 4.6000 & 12471 \\
\hline 4 & 6.9000 & 3165 \\
\hline 5 & 9.2000 & 1695 \\
\hline 6 & 11.5000 & 918 \\
\hline 7 & 13.8000 & 460 \\
\hline 8 & 16.1000 & 26 \\
\hline 9 & 18.4000 & 0 \\
\hline 10 & 20.7000 & 26 \\
\hline 11 & 23 & 0 \\
\hline
\end{tabular}

Figure 3.7 Distance distribution table

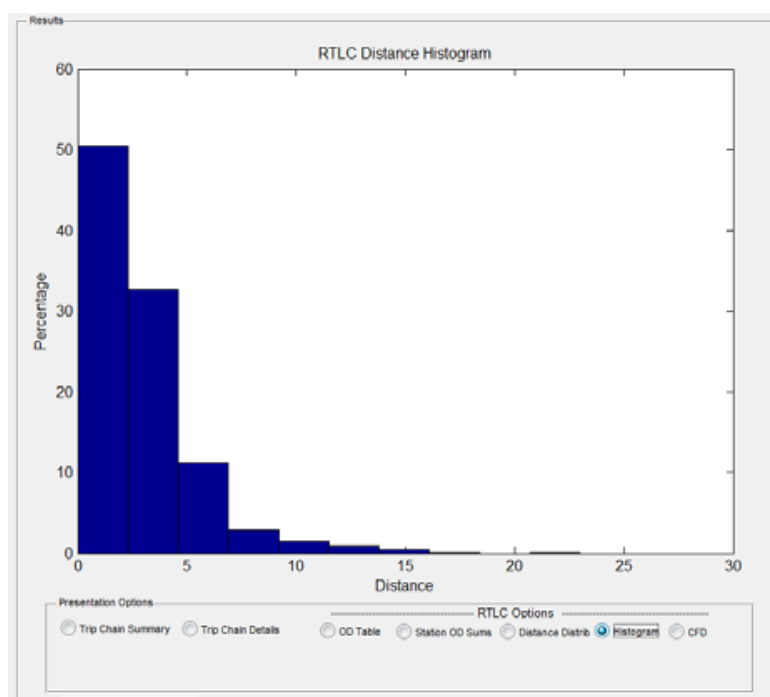

Figure 3.8 Sample normalize histogram view of the RTLC

Note that the implementation of the RTLC operates on station detection records, not on segment travel time records available in the CWS5200 segment data standard. This implementation exposed the need to define a station detection data standard, comparable to CWS5200, but applicable to individual detections at stations.

This report is intended as a reference document for the implementation of the RTLC for procurement of equipment and analytics related to re-identification data. The RTLC software, as described herein and detailed in "Arterial Trip Length Characteristics Software” [17], is available in BluSTATs version 2.25 and above. 


\section{Chapter 4: Forums}

During the course of this research project, a series of six forums were held in which the research team presented material to and interacted with practitioners. These forums took the format of interactive workshops. The goals were to introduce new data tools, educate attendees about how these could be used to assist in the management of arterial traffic control, and gather information about the needs, challenges, and preferences of the practitioners who are most likely to make use of these tools.

Figure 4.1 shows a map with the location and dates of the forums. The six forums were held in six different states in different parts of the US. They attracted over 300 attendees in total across all of the forums. This section presents an overview of each forum.

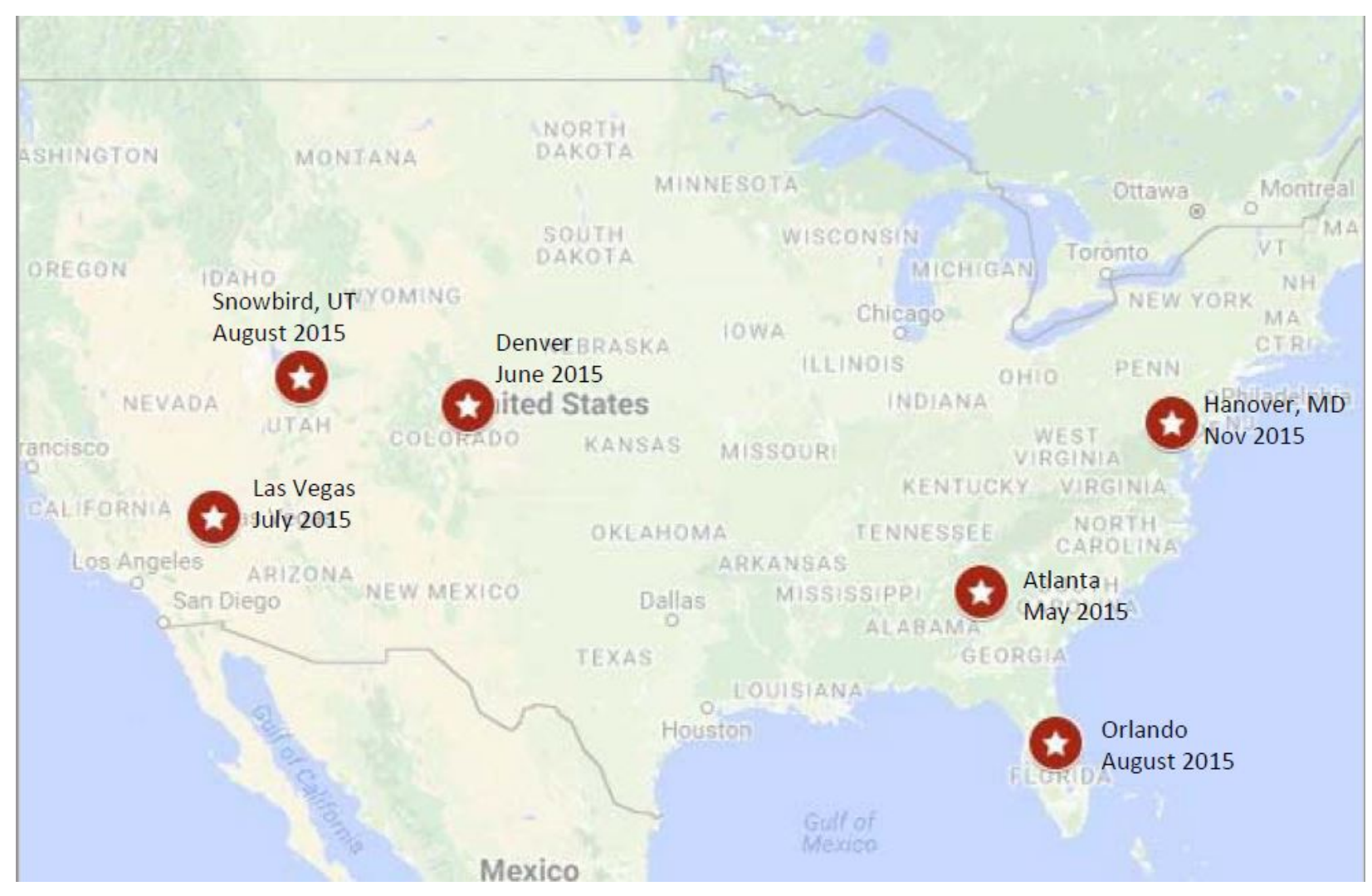

Figure 4.1 Forum Locations 


\subsection{Georgia Department of Transportation (Atlanta, GA)}

On May 13, 2015, Darcy Bullock presented a workshop behalf of the SBIR 3 project in Atlanta, Georgia. The event was hosted by Alan Davis of Georgia Department of Transportation at the offices of Atkins and was advertised through the website of the Georgia Section of the Institute of Transportation Engineers. Darcy also gave a brief presentation at the Georgia ITE monthly meeting held the following day. The workshop had a turnout of approximately 60 to 70 participants as seen in Figure 4.2.

This was a conversational style workshop. Though a great deal of material was covered, participation was strongly encouraged throughout the day and the attendees responded enthusiastically. The day's agenda is shown below in Table 4.1.

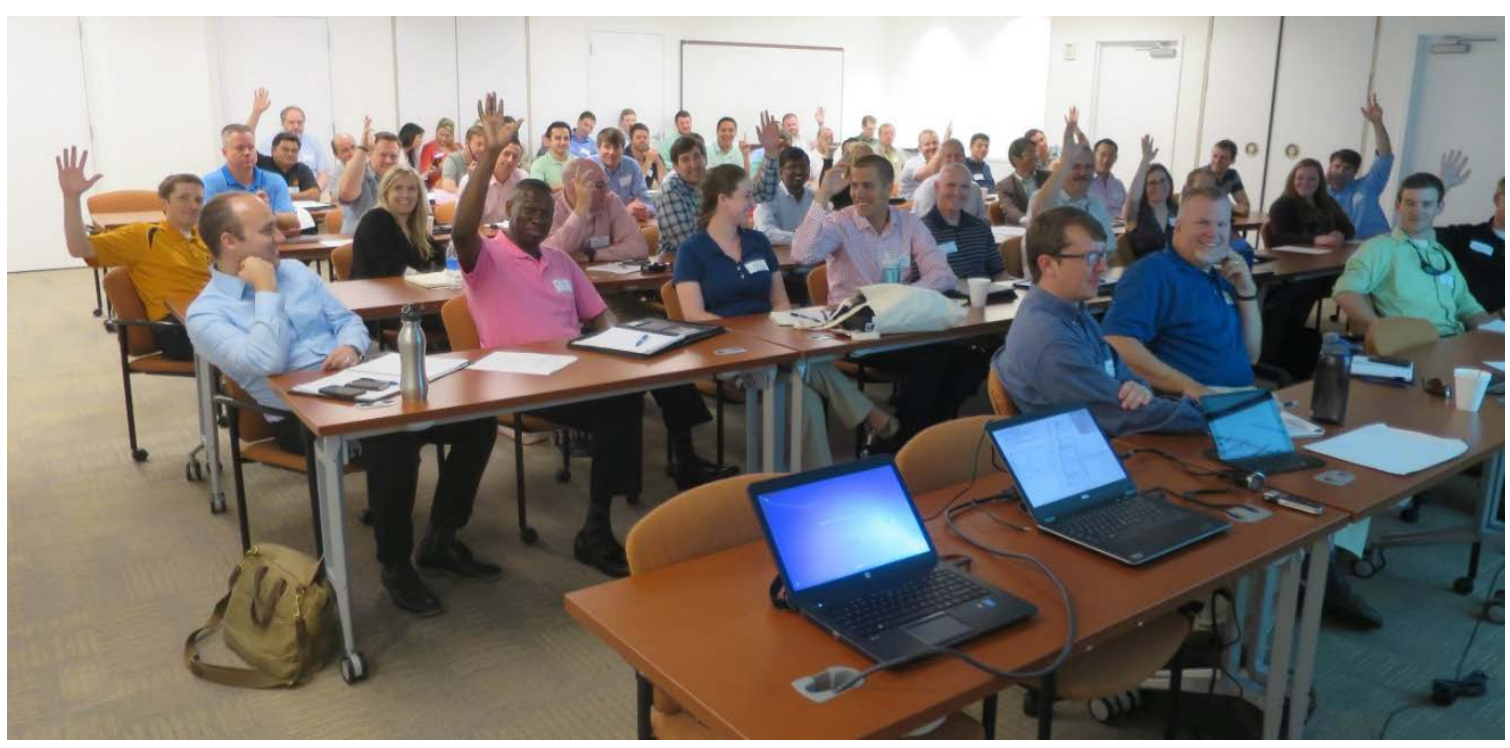

Figure 4.2 Workshop participants on May 13, 2015, Atlanta, Georgia

Table 4.1 Atlanta, GA workshop agenda

\begin{tabular}{|l|l|l|}
\hline 1. & \multicolumn{2}{l|}{ Survey } \\
\hline 2. & \multicolumn{2}{|l|}{ Introductory Remarks } \\
\hline & a. & Stan Young (Traffax) \\
\hline & b. & Alan Davis (GDOT) / Eddie Curtis (FHWA) \\
\hline 3. & Discussion Corridor \\
\hline & a. & Map \\
\hline & b. & VLC Video \\
\hline & c. & Dialog \\
\hline 4. & Basics of Probe Data Reduction \\
\hline & a. & Deployment \\
\hline & b. & How to tabulate BT Data CFD \\
\hline & c. & CFD \\
\hline 5. & Pyramid Perspective on Active Traffic Management \\
\hline 6. & PCD / Progression Concepts \\
\hline 7. & \multicolumn{2}{|l|}{ Split Failure Concepts } \\
\hline 8. & Future Directions \\
\hline 9. & Probe Data and Ranking Indexes \\
\hline 10. & Real Time Corridor Performance Measure Dashboards \\
\hline
\end{tabular}


The first item on the agenda for the day was to hand out a survey (shown in Table 4.2). This survey was administered prior to the workshop presentation and discussion to gauge the participants' assessment of the importance of traffic signal performance measures as well as their access to tools for performance measurement.

Attendees were asked to respond to the questions with the following scale:

$\begin{array}{ll}5 & \text { Strongly Agree } \\ 4 & \text { Agree } \\ 3 & \text { Neutral } \\ 2 & \text { Disagree } \\ 1 & \text { Strongly Disagree }\end{array}$

Figure 4.3 shows the distribution of public sector and consultant attendees at the workshop. Thirty-nine participants returned the survey, and the majority self-identified as consultants.

Table 4.3 shows the relationship between pairs of questions in the survey. Figure 4.4 shows the distribution of the responses. Of particular note is the approximately one point gap between what respondents believe are the performance measures they need to effectively manage their traffic signal system infrastructure and what they currently have available. Subsequent discussion throughout the day validated this perceived gap and the need for a technical document that agencies can use as a basis for procurement specifications.

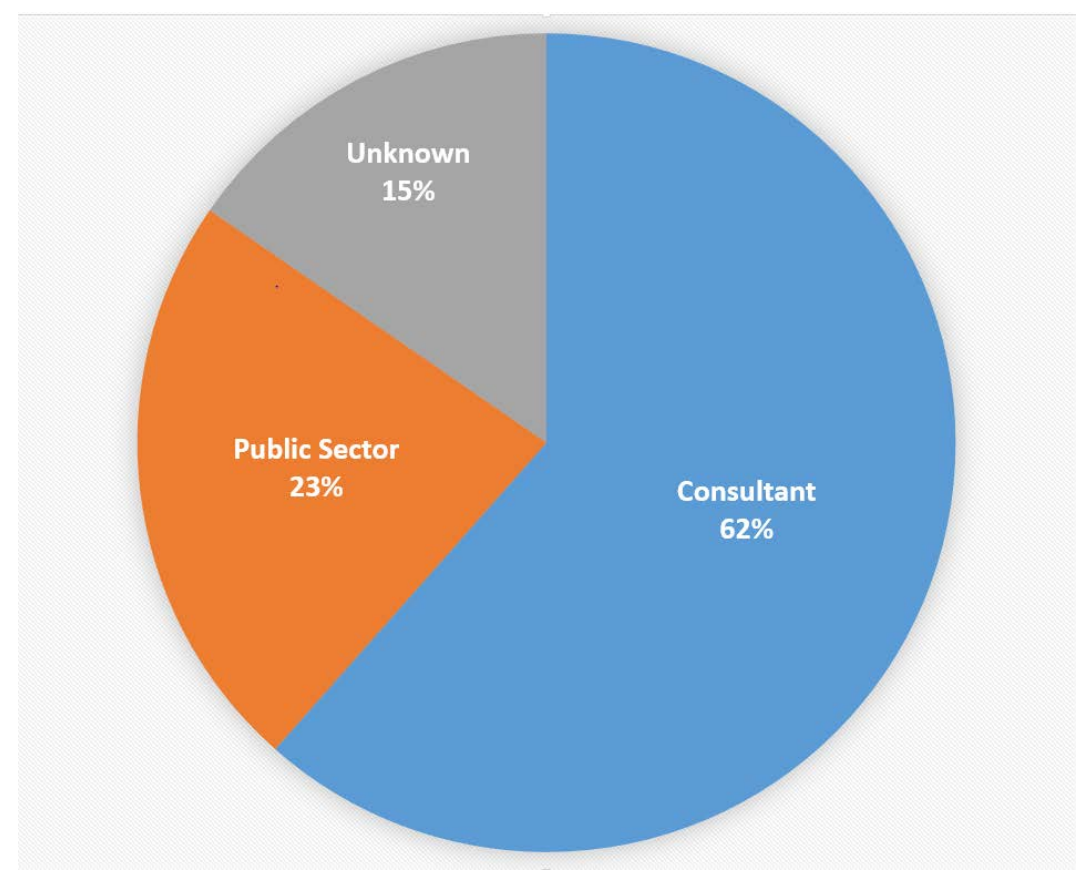

Figure 4.3 Distribution of attendee employment sectors 
Table 4.2 Workshop survey

The following performance measures are important in my daily professional activities:

\begin{tabular}{|c|c|c|c|c|c|c|}
\hline 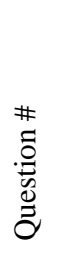 & 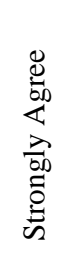 & 营 &  &  & 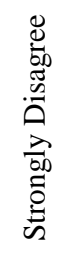 & \\
\hline 1. & & & & & & Travel Time \\
\hline 2. & & & & & & Travel Time Reliability \\
\hline 3. & & & & & & Quality of Progression (arrivals on green on an arterial) \\
\hline 4. & & & & & & Sufficient Green Time to serve vehicles waiting \\
\hline 5. & & & & & & \% of Detection that is working \\
\hline 6. & & & & & & $\%$ of Communication that is working \\
\hline
\end{tabular}

Please answer the questions below based upon what you have operational today.

\begin{tabular}{|c|c|c|c|c|c|c|}
\hline 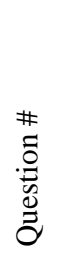 & 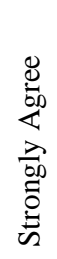 & 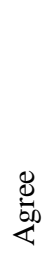 & 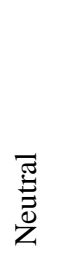 & 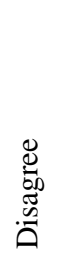 & 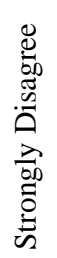 & \\
\hline 7. & & & & & & I believe I have access to good technology to measure travel time along corridors. \\
\hline 8. & & & & & & $\begin{array}{l}\text { I believe I have access to tools that let me quickly analyze corridor travel time trends and } \\
\text { travel time reliability. }\end{array}$ \\
\hline 9. & & & & & & $\begin{array}{l}\text { I believe I have access to data and tools to identify locations where there are } \\
\text { opportunities to improve progression between signalized intersections on a signalized } \\
\text { arterial. }\end{array}$ \\
\hline 10. & & & & & & $\begin{array}{l}\text { I believe I have access to data and tools to identify locations where I may need to adjust } \\
\text { the allocation of green time between competing movements. }\end{array}$ \\
\hline 11. & & & & & & I believe I have access to data and tools to identify vehicle detection maintenance issues. \\
\hline 12. & & & & & & $\begin{array}{l}\text { I believe I have access to data and tools to identify traffic signal communication } \\
\text { maintenance issues. }\end{array}$ \\
\hline 13. & & & & & & $\begin{array}{l}\text { I believe I have access to data and tools to quickly perform before/after assessment of } \\
\text { traffic signal timing and improvement initiatives }\end{array}$ \\
\hline
\end{tabular}

14. Which of the following best describes your employer

a. Consultant

b. Public Sector

c. Vendor 
Table 4.3 Paired survey questions

\begin{tabular}{|l|l|}
\hline $\begin{array}{l}\text { Questions regarding importance of Performance } \\
\text { Measures }\end{array}$ & $\begin{array}{l}\text { Corresponding question regarding access to } \\
\text { performance measure tools }\end{array}$ \\
\hline 1. Travel Time & $\begin{array}{l}\text { 7. I believe I have access to good technology to } \\
\text { measure travel time along corridors. }\end{array}$ \\
\hline 2. Travel Time Reliability & $\begin{array}{l}\text { 8. I believe I have access to tools that let me quickly } \\
\text { analyze corridor travel time trends and travel time } \\
\text { reliability. }\end{array}$ \\
\hline $\begin{array}{l}\text { 3. Quality of Progression (arrivals on green on an } \\
\text { arterial) }\end{array}$ & $\begin{array}{l}\text { 9. I believe I have access to data and tools to identify } \\
\text { locations where there are opportunities to improve } \\
\text { progression between signalized intersections on a } \\
\text { signalized arterial. }\end{array}$ \\
\hline 4. Sufficient Green Time to serve vehicles waiting & $\begin{array}{l}\text { 10. I believe I have access to data and tools to identify } \\
\text { locations where I may need to adjust the allocation of } \\
\text { green time between competing movements. }\end{array}$ \\
\hline 5. \% of Detection that is working & $\begin{array}{l}\text { 11. I believe I have access to data and tools to identify } \\
\text { vehicle detection maintenance issues. }\end{array}$ \\
\hline 6. \% of Communication that is working & $\begin{array}{l}\text { 12. I believe I have access to data and tools to identify } \\
\text { traffic signal communication maintenance issues. }\end{array}$ \\
\hline
\end{tabular}

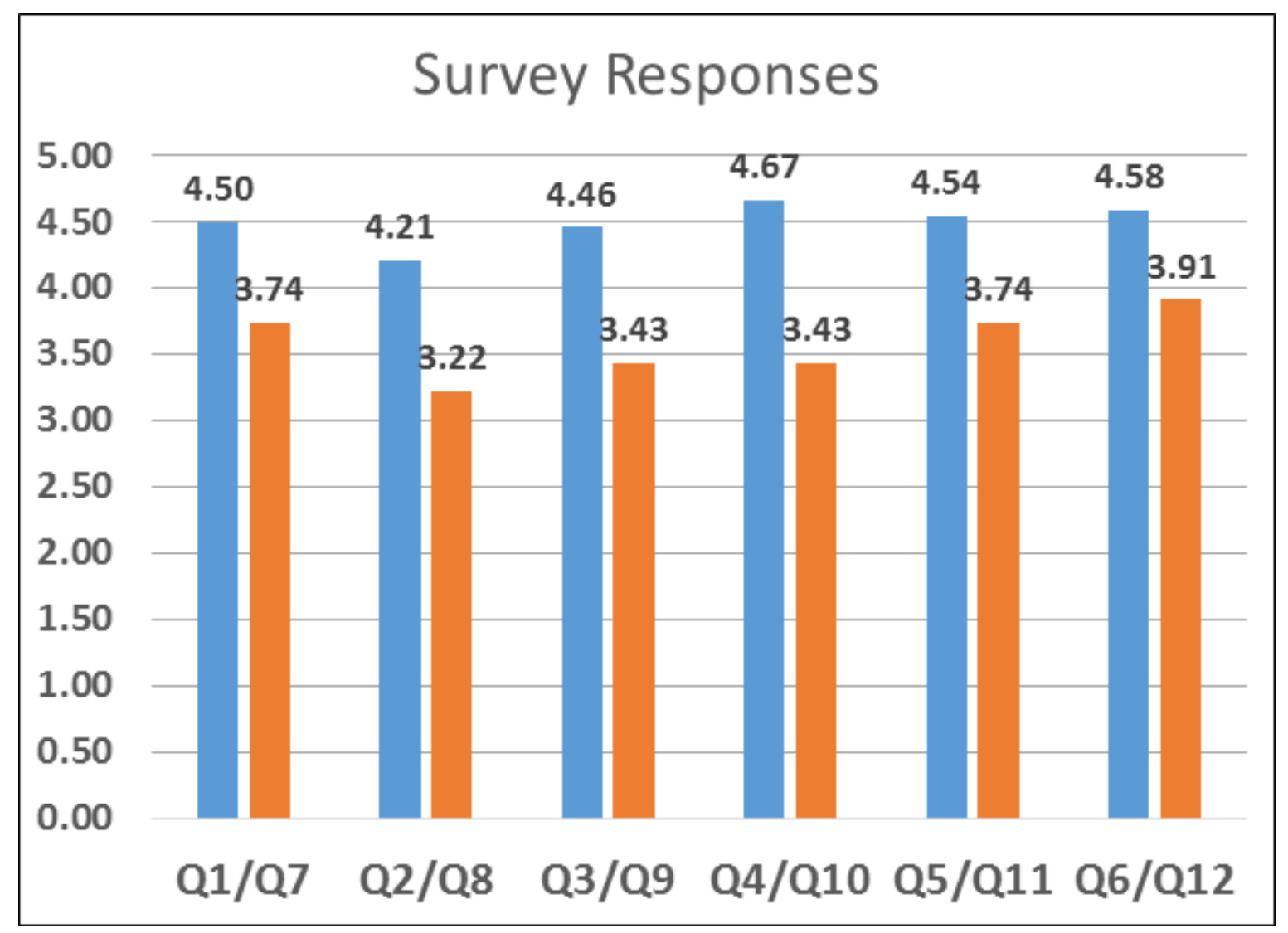

Figure 4.4 Results of survey questions 
The following summarizing some the key dialog obtained from participants during the workshop:

- Effective arterial management requires a diverse set of data

o High resolution traffic signal data to characterize arrivals on green and split failures

o Probe data to characterize arterial travel time

o Infrastructure health data to verify that communication and detection is functioning properly is important.

- The Atlanta area has a relatively large deployed base of Bluetooth sensors for obtaining probe vehicle travel time information.

- When Bluetooth sensors are deployed, mid-block deployment is the preferred location. However, due to power and communication costs, they are frequently deployed at signalized intersections. This necessitates using slightly more complex data reduction algorithms to reduce noise in the data set.

- Using probe data to measure before/after changes in travel time is a very effective tool for communicating the impact to decision makers, particularly when it is combined with AADT to estimate savings in user costs.

- Georgia is aggressively deploying high resolution data collection capabilities at its signalized intersections.

- Georgia consultants generally spend considerable time in the field tweaking Synchro output. This is found to be very beneficial, but costly to scale to all TOD plans, particularly Saturday and Sunday plans.

- There is mixed feeling on the utility of adaptive control. However, all agreed that both hiresolution signal data and probe data based performance measures are critical to objectively assess the impact of adaptive control.

- Although not unanimous, there was general consensus that the consultant model will likely evolve from the current practice of:

o Obtain turning movement counts

o Run Synchro

o Implement Timing plans

o Assess impact Synchro

To the following practices:

o Use probe data to prioritize locations for deploying high resolution data logging equipment

o Use high resolution data instead of count data/Synchro to identify opportunities to improve green allocation and offsets

o Assess outcome with both high res data and probe data

o Report quantitative improvement data to decision makers to demonstrate impact their investments are having in signal system management. 


\subsection{International Performance Measurement and Data Conference (Denver, CO)}

On June 2, 2015, Stan Young presented an overview of the SBIR III project at the $5^{\text {th }}$ International Transportation Systems Performance Measurement and Data Conference held in Denver, Colorado. This two day event brought together US and international experts to address developing, applying, and delivering performance measures to support transportation decisions. The conference was structured with plenary sessions, and breakout sessions in four thematic areas with respect to performance measures: Driving Decision, Tracking the Moves, Data Web, and State of the Practice.

Stan Young hosted a discussion on 'Monitoring and Assessing Arterial Traffic Performance' as part of one of the Data Web breakout sessions. About 300 people attended the overall conference. The breakout session included 30 to 50 people.

Two other presenters in this breakout session gave talks on 'Florida's Approach to Maximizing Advances in Data and Technology for Performance Management' and 'Reinventing the MPO Performance Monitoring Process in the Era of Interactive Data Visualizations'. The titles of the co-presenters presentations were indicative of the interests of the majority of the attendees, most had broad management responsibilities to organize data and report on a variety of transportation related items ranging from pavement condition, to safety, to congestion. This audience makeup is in contrast to that of the recently completed George workshop in which about 70 highly focused traffic engineering professionals discussed detailed aspects of the proposed measures and the enabling technology.

The presentation combined an overview of outsourced probe data fidelity assessed at the University of Maryland, and then used that as a bridge to begin to discuss the need for more standardized, broad-based arterial performance measures as explored in the SBIR III project, and enabled by re-identification data and High Resolution Probe data. The agenda is shown in Table 4.4.

The following PPT file was used for the presentation:

- $\quad$ https://app.box.com/s/c45fa22zr5l16rp49in98j1wxsqr2uvk

This crowd was interested in understanding top level metrics and how / whether they appropriately reflect the condition of the arterial system as a whole. For example, one question raised was whether the percent of arrivals on green and likelihood of split failures were too intersection specific and granular to use for broad-based performance reporting.

Material related to creating a common language to facilitate communications between operations, planning (typically responsible for system level performance reporting), and traffic engineers was well received. The four main (or proposed) metrics of travel time, reliability, percent arrival on green, and split failures provided a concise and easily communicable performance criteria - not only with the public and elected officials, but also to streamline internal communications within DOTs, city agencies, and MPOs to better advance the mission of the agency.

The proposed reliability measure for signal controlled arterials was discussed briefly in response to the question of the preferred reliability metric for arterials. The slope formed from the line adjoining the 15th and 85th percentile travel times was proposed.

Final Report

Contract \#: DTFH61-14-C-00035 
Lastly, the presentation emphasized the need to address signal controlled arterial performance measures distinct from the performance measures that have been adopted for freeways. The audience readily acknowledged this point as most have come to realize that freeway measures are not only difficult to apply to arterials, but are also inadequate in many respects to portray the true performance of the arterial management system.

Table 4.4 Presentation Outline

\begin{tabular}{|l|l|l|}
\hline 1. & \multicolumn{2}{|c|}{ Traffic Data - Fidelity of Outsource Probe Data } \\
\hline & a. & Where we have been \\
\hline & b. & Where we are now \\
\hline 2. & Completing the Picture with Arterial Performance Measures \\
\hline & a. & Bringing in Volume Data - Statewide \\
\hline & b. & Extending Real-Time to Arterial Networks \\
\hline & c. & Arterial Management Systems \\
\hline
\end{tabular}




\subsection{ITE Western District Annual Meeting (Las Vegas, NV)}

On July 20, 2015, Chris Day (Purdue University) presented a workshop at the ITE Western District Annual meeting in Las Vegas, Nevada. The workshop was included in the program for the meeting (Figure 4.5) and competed with three other technical sessions and other events that were scheduled in parallel. There were approximately 20 attendees.

This workshop was presented as a technical podium session. The total time allotted for the workshop was 90 minutes. About one hour was used to present material and the remaining 30 minutes were used for audience questions. Audience questions and discussion filled the entire 30 minutes. Table 4.5 shows a list of topics covered in the workshop.

\section{Preliminary Technical Session Schedule}

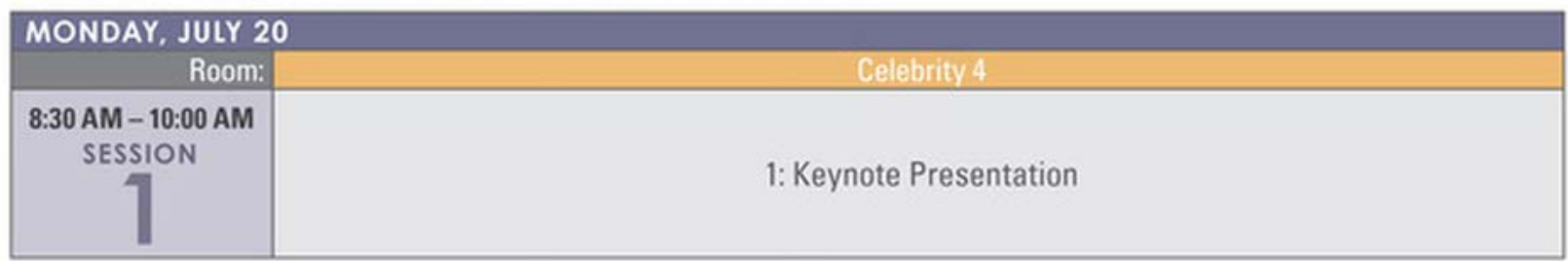

\begin{tabular}{|c|c|c|c|c|}
\hline TRACK: & \begin{tabular}{l|l} 
A SAFETY \& \\
MULTI-MODAL
\end{tabular} & \begin{tabular}{l|l} 
B PLANNING \& \\
TECHNOLOGY
\end{tabular} & C OPERATIONS & D WORKSHOPS \\
\hline Room: & Wilshire A & Wilshire B & Sunset 3-4 & Sunset 5-6 \\
\hline $\begin{array}{c}\text { 10:30 AM - 12:00 PM } \\
\text { SESSION }\end{array}$ & $\begin{array}{l}\text { 2A: Complete } \\
\text { Streets }\end{array}$ & $\begin{array}{l}\text { 2B: Effective } \\
\text { Tools in Data } \\
\text { Collection }\end{array}$ & $\begin{array}{l}\text { 2C: Traffic Signal } \\
\text { Advancements }\end{array}$ & $\begin{array}{l}\text { 2D: Workshop - The World of } \\
\text { Mobility is Changing: What are } \\
\text { YOU Doing About It? }\end{array}$ \\
\hline $\begin{array}{c}\text { 1:30 PM-3:00 PM } \\
\text { SESSION }\end{array}$ & $\begin{array}{l}\text { 3A: Bicycle } \\
\text { Facility Planning } \\
\text { \& Design }\end{array}$ & $\begin{array}{l}\text { 3B: Corridor } \\
\text { Management }\end{array}$ & $\begin{array}{l}\text { 3C: Traffic } \\
\text { Management }\end{array}$ & $\begin{array}{l}\text { 3D: Workshop - California's SB } \\
743 \text { - Using VMT as a Performance } \\
\text { Measure for Transportation Studies }\end{array}$ \\
\hline $\begin{array}{c}\text { 3:30 PM - 5:00 PM } \\
\text { SESSION }\end{array}$ & $\begin{array}{l}\text { 4A: Safety } \\
\text { Analysis \& } \\
\text { Planning }\end{array}$ & $\begin{array}{l}\text { 4B: } \\
\text { Transportation } \\
\text { Planning Tools }\end{array}$ & $\begin{array}{l}\text { 4C: Lessons } \\
\text { Learned in Traffic } \\
\text { Operations }\end{array}$ & $\begin{array}{l}\text { 4D: Arterial Performance } \\
\text { Management Workshop }\end{array}$ \\
\hline
\end{tabular}

Figure 4.5 Listing of the Arterial Performance Management Workshop (Session 4D) in the ITE Western District program for Monday, July 20 events. 
Table 4.5 Las Vegas, NV workshop topics

\begin{tabular}{|c|c|c|}
\hline 1. & \multicolumn{2}{|c|}{ Background and objectives } \\
\hline 2. & \multicolumn{2}{|r|}{ Motivation for performance measurement } \\
\hline 3. & \multicolumn{2}{|r|}{ State of the practice and system requirements } \\
\hline \multirow[t]{4}{*}{4.} & \multicolumn{2}{|c|}{ Evaluation of arterial travel times } \\
\hline & a. & Introduction to probe data \\
\hline & b. & How to visualize data (raw data and CFDs) \\
\hline & C. & Example of a before/after scenario \\
\hline \multirow[t]{4}{*}{5.} & \multicolumn{2}{|c|}{ Signal performance measures } \\
\hline & a. & Brief recap of signal control concepts \\
\hline & b. & Introduction to high resolution data \\
\hline & C. & $\begin{array}{l}\begin{array}{l}\text { Example use of high resolution data for simple } \\
\text { diagnostics }\end{array} \\
\end{array}$ \\
\hline \multirow[t]{5}{*}{6.} & \multicolumn{2}{|r|}{ Evaluation of Arterial Progression } \\
\hline & a. & Possible causes of progression deficiencies \\
\hline & b. & Visualization of high resolution data using PCD \\
\hline & C. & Corridor before/after example \\
\hline & d. & Outcome assessment using probe vehicle data \\
\hline \multirow[t]{5}{*}{7.} & \multicolumn{2}{|c|}{ Evaluation of Capacity Utilization } \\
\hline & a. & $\begin{array}{l}\begin{array}{l}\text { Examples of under-saturated and oversaturated } \\
\text { movements }\end{array} \\
\end{array}$ \\
\hline & b. & ROR and GOR as metric for identifying split failure \\
\hline & C. & Use of ROR/GOR for before/after comparison \\
\hline & d. & Agency-wide and longitudinal analysis \\
\hline 8. & \multicolumn{2}{|c|}{ Signal maintenance } \\
\hline & a. & Scope of problem \\
\hline & b. & Classifying vehicle detector errors \\
\hline & C. & Quantified relative impact of maintenance \\
\hline 9. & \multicolumn{2}{|c|}{ Recap, future directions, and questions } \\
\hline
\end{tabular}


The following summarizes participant input and questions during the workshop.

- The value of good equipment maintenance and validating public complaint calls resonated well with the audience.

- A participant asked how the performance measures would appear with video detection rather than the typical INDOT loop detection used for the data shown in the presentation. The response was that, as long as the quality of the detection was the same, the performance measures should have the same appearance and that, essentially, the performance measures are intended to be independent from the specific detector technology.

- A participant asked about INDOT's use of four-channel count and presence loop amplifier cards.

- A participant asked about some specific details about one vendor's implementation of the high resolution data. Essentially, it was asked how to enable the data and how it could be harvested. In response, the necessary steps to set up data collection were explained. Essentially, the following was explained. The high resolution data is collected on the controller independently of any central system management software, but has to be enabled and retrieved. Central system management software can assist enabling and retrieving the data but is not required. It was pointed out that several vendors were now offering high resolution data collection in their controller products.

- The question was asked about whether INDOT tended to focus its attention on peak or off peak operations. The response was that both were of interest, but in many cases it is the off-peaks where the spare capacity exists to address problems.

- A participant commented on a slide that was presented in which the rate of split failures were shown by data to be higher during the midday than during the other times of day. This engineer's experience was that midday was becoming the most difficult time of day to design signal timing for, because there was no dominant direction in the traffic flow. Changing activity patterns as the population ages were thought to be driving these trends. The participant asked if the researchers had seen other evidence of this phenomenon. The response was that the data that inspired the comment was probably the best example, and that the insights provided a helpful explanation for why there were more midday split failures than we otherwise might have expected. 


\subsection{NRITS Conference (Snowbird, UT) \& FLTDS (Orlando, FL)}

On August 10, 2015, Stan Young presented 'Monitoring and Assessing Arterial Traffic Performance', which included an overview of the SBIR III project emphasis at the National Rural Intelligent Transportation System (NRITS) Conference in Snowbird, Utah. This three day event brought together primarily US interests in rural ITS deployment and operations. Total attendance was approximately 200.

On August 19, 2015 Stan Young presented the same presentation at the Florida Transportation Data Symposium (FLTDS) in Orlando, Florida. This three day event is sponsored by the Florida Department of Transportation, and attracts planning, traffic and operations personnel from all of Florida as well as their industry partners, and representatives from other states. Total attendance was approximately 400.

Both events were organized with plenary sessions followed by a topical breakout. At the NRITS conference, Dr. Young presented in a breakout session along with Mark Taylor from Utah DOT. Mark was instrumental in implementing a software reporting system for high-resolution controller data throughout Utah. At the FLTDS, Dr. Young presented in a session that included Anita Vandervalk from Cambridge Systematics and Michael Pack from University of Maryland Center for Advanced Transportation Technology. Anita presented on an arterial performance initiative in Los Angeles and Michael presented on the Regional Integrated Transportation Information System which has performance measures tools for a variety of freeway and arterial applications.

At both events, Dr. Young presented and hosted a discussion on 'Monitoring and Assessing Arterial Traffic Performance' within the respective breakout sessions.

Dr. Young's presentation opened with an overview of outsourced probe data fidelity assessed at the University of Maryland both for freeways and arterials, and then bridged to a discussion on the need for more standardized, broad-based arterial performance measures as explored in the SBIR III project, enabled by re-identification data and High Resolution Probe data. The presentation covered four proposed measures (travel time, travel time reliability, percent stopped on red, and intersection capacity), as well as the need for a common language to elevate the needs for optimized signal management within transportation, and to begin to establish a mature management system for arterials like those already in place for other infrastructure systems such as roads and bridges. The outline for the presentation is shown in Table 4.6.

At the FLTDS, the presentation led to significant discussion. Highlights included:

- The Florida division of statistics recently obtained probe based data and has been informally evaluating it for accuracy. Observations include an apparent over-reporting of speed in urban areas, and under-reporting on rural freeways. Results on arterials are preliminary, but appear to agree with those presented.

- Cambridge Systematics is assisting Maryland SHA on the implementation of SHRP products that emphasize the use of travel time reliability in design, and then measured in operation. The concepts presented for arterials were discussed within the context of the SHRP implementation. Dr. Young also suggested inviting the community working on this implementation to the upcoming workshop in Maryland.

- Several questions related to the clarification and more in-depth assessment of probe data were asked and discussed subsequent to the presentation. One question solicited the critical issues for probe data to evolve to more accurate arterial travel times. The response identified three items: (1) continued increased probe density, (2) evolving to point-pair processing rather than the now

Final Report

Contract \#: DTFH61-14-C-00035 
dominant point speed processing, (3) reporting more than just the central tendency in order to capture the complex travel time patterns. However, at this time no one is sure what those other metrics are.

- The director of the Office of Traffic Engineering and Operations for FDOT chaired the session. Discussion after the session indicated an eagerness to host a workshop in Florida similar to the one hosted in Georgia (and soon to be Maryland).

- The audience was a mix of traffic engineers, ITS operations, and planning personnel. It was the most balanced audience to date in Dr. Young's opinion. The audience stayed engaged, and receptive to the Arterial Management emphasis, and not just the technology discussion.

- Gridsmart, a startup in this area, attended the conference and the session. This is evidence of some commercial activity in the area.

- Mohammed Hadi from Florida International University attended the presentation, and later engaged in questions about the statistical models of Bluetooth for use in traffic simulation tools such as VISSUM. This is similar to the concept David Gibson proposed for an SBIR IIB initiative.

At NRITS, the presentation complemented the material presented by Mark Taylor. However, there were minimal questions and discussion.

Table 4.6 Presentation Outline

\begin{tabular}{|l|l|l|}
\hline 1. & \multicolumn{3}{|l|}{ Traffic Data - Fidelity of Outsource Probe Data } \\
\hline & a. & Freeways with multiple vendors \\
\hline & b. & Arterial data quality challenges \\
\hline 2. & Completing the Picture with Arterial Performance Measures \\
\hline & a. & Re-identification and High-Res Controller data \\
\hline & b. & Four key measures from these sources \\
\hline & c. & Growing toward Arterial Management Systems \\
\hline 3. & \multicolumn{2}{|c|}{ Announcement for volume based probe initiative } \\
\hline
\end{tabular}




\subsection{Maryland ITS (Hanover, MD)}

On November 10, 2015, Stan Young (Traffax), Darcy Bullock (Purdue University), and Chris Day (Purdue University) presented a workshop at the Maryland State Highway Administration facility in Hanover, MD. The event was hosted by the Intelligent Transportation Society of Maryland and was organized by Diederick VanDillen of Jacobs Engineering Group. There were approximately 60 attendees (Figure 4.6, Figure 4.7).

This workshop was presented as a series of presentations in a 2-hour morning session and a 2.5-hour afternoon session. This was a conversational style workshop and there was active audience participation as well as time for individual discussions with the presenters throughout the day. The program opened with an introduction of the presenters by Diederick VanDillen and a short history of the work and Traffax-Purdue collaboration given by Peter Carnes (Traffax). Stan Young then presented an overview of the work being done under this SBIR III project followed by a summary of the use of re-identification and probe data in assessing arterial performance.

After a break, Darcy Bullock presented background material on high resolution data and gave an overview with several brief examples of using high resolution data in tandem with probe data. After breaking for lunch, Chris Day presented material on integrating high resolution controller data and probe data using the example of signal progression. Darcy Bullock followed this up with some additional case study discussion including an example of capacity utilization and origin-destination analysis with probe data. Finally, Stan Young presented some closing words to wrap up the workshop. Table 4.7 shows the day's agenda. 


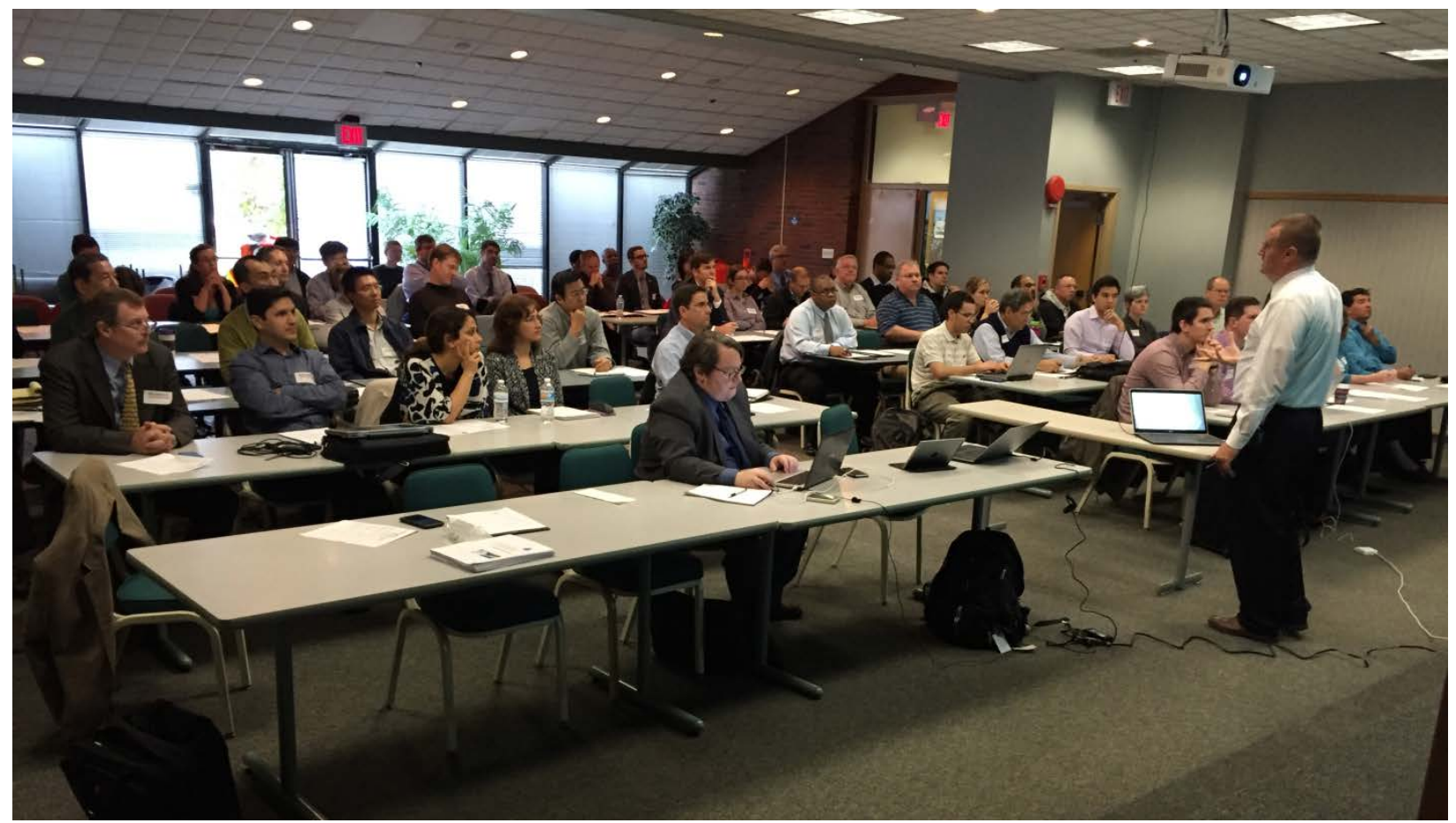

Figure 4.6 Stan Young speaking at the ITS Maryland workshop, November 10, 2015.

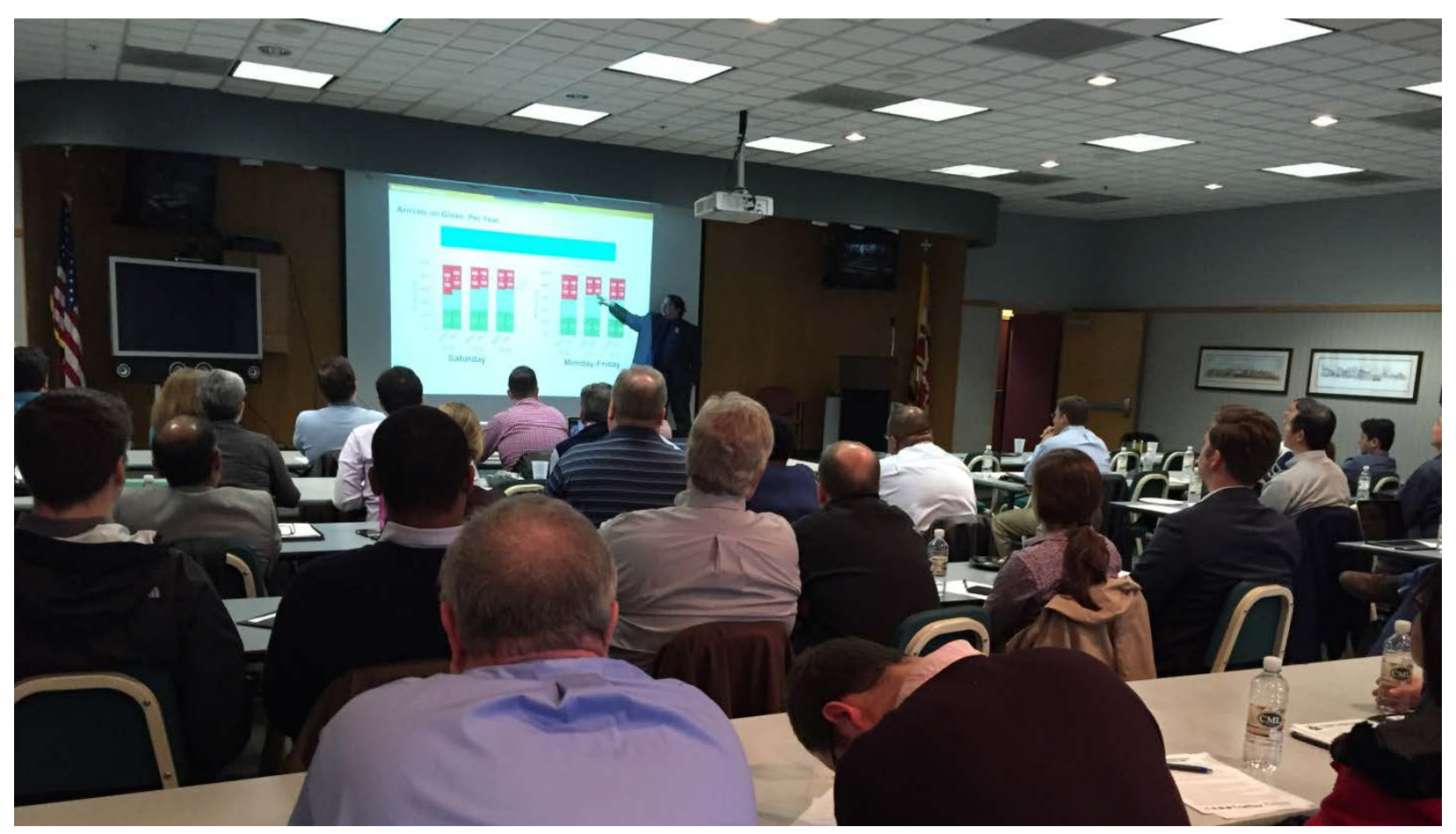

Figure 4.7 Chris Day speaking at the ITS Maryland workshop, November 10, 2015. 
Table 4.7 Hanover, MD workshop agenda

\begin{tabular}{|c|c|c|}
\hline 1. & \multicolumn{2}{|c|}{ Introductions (VanDillen) } \\
\hline 2. & \multicolumn{2}{|c|}{ Opening Words (Carnes) } \\
\hline 3. & \multicolumn{2}{|c|}{ “50,000 Foot View” (Young) } \\
\hline & \multicolumn{2}{|c|}{ Break } \\
\hline \multirow[t]{3}{*}{4.} & \multicolumn{2}{|r|}{$\begin{array}{l}\text { Vehicle Probe Data Collection \& Performance Measures } \\
\text { Methodology (Young) }\end{array}$} \\
\hline & a. & Monitoring and Assessing Arterial Traffic Performance \\
\hline & b. & Use of Outsourced Probe Data \\
\hline \multirow[t]{9}{*}{5.} & \multicolumn{2}{|r|}{$\begin{array}{l}\text { Integration of High Resolution Controller Data and Probe Data } \\
\text { (Bullock) }\end{array}$} \\
\hline & a. & Research perspective \\
\hline & b. & Probe data applications to arterial networks \\
\hline & C. & Hierarchy of signal system needs \\
\hline & d. & Relationship to pooled fund study \\
\hline & e. & $\begin{array}{l}\text { High level view on use of high resolution data to identify } \\
\text { where to improve operations }\end{array}$ \\
\hline & f. & $\begin{array}{l}\text { High level view on use of probe data to communicate to } \\
\text { decision makers }\end{array}$ \\
\hline & g. & Future vision - less modeling and more measuring \\
\hline & \multicolumn{2}{|c|}{ Lunch } \\
\hline \multirow[t]{9}{*}{6.} & \multicolumn{2}{|r|}{$\begin{array}{l}\text { High Resolution Data Performance Measures to Support } \\
\text { Active Traffic Management (Day) } \\
\end{array}$} \\
\hline & a. & Development of performance measures \\
\hline & b. & $\begin{array}{l}\text { How probe data tells us where problems are-but not } \\
\text { necessarily the causes }\end{array}$ \\
\hline & C. & $\begin{array}{l}\text { Introduction to Purdue Coordination Diagram and how to } \\
\text { use it }\end{array}$ \\
\hline & d. & Offset optimization based on high resolution data \\
\hline & e. & $\begin{array}{l}\text { Outcome assessment of offset optimization with probe } \\
\text { data }\end{array}$ \\
\hline & f. & $\begin{array}{l}\text { Monetization of user cost reductions-examples from a } \\
\text { 5-year longitudinal study }\end{array}$ \\
\hline & g. & Implementation \\
\hline & \multicolumn{2}{|c|}{ Break } \\
\hline \multirow[t]{4}{*}{7.} & \multicolumn{2}{|r|}{$\begin{array}{l}\text { Case Studies: Integration and Application of Performance } \\
\text { Measures }\end{array}$} \\
\hline & a. & $\begin{array}{l}\text { Use of Red Occupancy Ratio / Green Occupancy Ratio to } \\
\text { evaluate capacity utilization }\end{array}$ \\
\hline & b. & $\begin{array}{l}\text { Longitudinal comparison over wide temporally and } \\
\text { spatially distributed data }\end{array}$ \\
\hline & C. & $\begin{array}{l}\text { Use of probe data to separate fact from fiction-example } \\
\text { from I-65 diversion }\end{array}$ \\
\hline 8. & \multicolumn{2}{|c|}{ Closing Words (Young) } \\
\hline
\end{tabular}


The following summarizes some of the key dialog with participants:

- Participants asked about accuracy of probe data for arterials. The presentation showed that probe data from commercial sources (INRIX, HERE, TomTom) can be used to show proportional improvement for before and after studies but caution should be used for long term longitudinal studies. As probe data improves, long term analysis may show worsening performance but this may only be reflecting improved probe data accuracy (i.e. addressing the positive speed bias during congestion). It was also stated that the validation of probe data through the I-95 Corridor Coalition will continue.

- Regarding the evaluation of probe data accuracy by comparison with re-identification data, a participant asked how re-identification data was itself ground truth. The response was that the accuracy of re-identification travel times has been compared to travel times calculated using other sources such as toll tag data and was shown to have equivalent means and distributions.

- Participants asked about the difference between Bluetooth and Wi-Fi detection. The response was that Wi-Fi provides significantly more detections (15\% to $25 \%$ of the Traffax streams) but it is sensitive to the speed of traffic. As traffic speed increases, the probability of detection decreases. Use of Wi-Fi and Bluetooth data are complimentary and recommended to be used in tandem.

- A participant commented that the spatial resolution of outsourced probe data needs to improve to provide similar performance as Bluetooth/WiFi data collection technology.

- The notion that performance measures can be good indicators of what generates phone calls seemed to strongly capture audience interest.

- A goal of statewide performance reporting was stated as an important place to get to with automated performance measures.

- The automatic triggering of alarms based on the performance data was mentioned as a potential application of the data. For example, a potential trigger would be a certain number of performance measures exceeding a defined threshold over a given time period.

- An audience member pointed out that it is important to understand the performance of a system throughout the year. For example, do "summer" timing plans still operate well during the fall? And so on.

- An audience member mentioned that the workshop presented topics that were mostly vehicle focused and that it is important to include pedestrians in performance analysis. The response was that this was important, and while the workshop focused mainly on the vehicle mode, pedestrianfocused performance measures have been previously demonstrated using high resolution data.

- Several audience members pointed out the importance of having good context for stating user costs. There were numerous audience questions regarding the assumptions made to tabulate these costs. Additionally, there was audience interest in knowing whether similar information for the environmental costs could also be derived from the same analysis.

- Some adaptive control systems can be designed to encompass the "coordination" and "timing" aspects of the signal system hierarchy of needs. However, that does not necessarily show the value added by those systems. Comparison of their performance against a true "do-nothing" scenario is a poor perspective to determine value added. Instead, the performance of those systems to better handle their intended use cases - more dynamic response to changing conditions - would be a better basis of evaluation. This would develop understanding of where advanced control systems can be used to their best advantage, rather than installing them where they may provide less return on investment. There is likely potential to use performance measures to help facilitate the FHWA systems engineering approach. 
- Some discussion was focused on uses of both types of data in an integrated fashion. It was pointed out that high resolution controller data and probe data are both needed. High resolution data provides the tools to assess and address the signal timing issues at each intersection while reidentification and probe data provide the user experience directly. Re-identification and probe data should be used to document before and after conditions so that travel time and user costs can be objectively quantified.

- The message of "measure, not model" seemed to be well received. The key message was that tools presented during the workshop allow for direct measurement of many performance objectives. Models based on one day counts no longer need to be relied upon. Specific examples touched upon in the workshop include use of ROR/GOR for evaluating capacity utilization, or PCDs and re-identification / probe data travel times (particularly cumulative frequency diagrams showing their distribution) to evaluate progression. These fit into an overall hierarchy of needs for operating the system. 


\section{Chapter 5: Conclusions and Future Development}

The deliverables produced in this SBIR project explored the potential of recently developed traffic data sets, vehicle re-identification data and high-resolution data from signal controllers, to enable new methods of data-driven arterial management. This final report provided an overview of these data sources in Chapter 1, and summarized the content of the deliverables, with Chapter 2 giving a synopsis of the five reports and two case studies, Chapter 3 describing the implementation tools, including a data standard and software products of this project, and Chapter 4 summarizing the public forums conducted by the research team as part of this project.

At the time this final report was written, the arterial management concepts being explored in this project have become increasingly relevant as agencies endeavor to integrate the new sources into their operations. Many agencies have invested in systems to measure travel times with vehicle re-identification, while private sector data vendors have introduced similar data products based on analysis of GPS-enabled mobile devices. Meanwhile, the six major manufacturers of traffic signal controllers in the US have all adopted the high-resolution data concept and are now offering this feature in at least one newer controller model. This chapter outlines the near and long term prospects of this technology in light of these developments.

\subsection{Vehicle Re-Identification Data and Emerging Data Sets}

Automated vehicle re-identification continues to be a useful traffic engineering tool. Bluetooth MAC address matching, which began to proliferate rapidly about 10 years ago, continues to be a valuable data source. In recent years it has been augmented with the addition of Wi-Fi network detection in response to evolving consumer preferences for wireless communication and, given that mobile wireless devices are unlikely to decline in popularity in the near future, the potential for automatic vehicle re-identification remains strong.

This stream of technology could be considered the first generation of scalable automated vehicle reidentification methods. At the time of writing, there are two potential candidates for what might become a second generation, one based in the private sector and the other in the public sector. These new forms are presented here both as an indication of possible future data collection technology and to understand that the fundamental improvements introduced through re-identification technology, namely the ability to characterize performance using travel time distribution data, is not limited to re-identification technology. The fundamental enhancements demonstrated through application of travel time overlay plots and cumulative frequency distributions are equally valid when applied to the data from these emerging technologies.

- Private sector data providers have been harvesting GPS location data from a variety of mobile devices for the past several years. This resource has been the basis of several new and evolving data products, the main one being minute-by-minute speeds for predefined roadway segments. The "raw" data on which those speeds are based can potentially provide a wealth of information. The GPS traces of individual devices, which are roughly equivalent to the data from floating car studies and does not require field infrastructure to obtain, can be processed to obtain travel times for individual paths. However, the data, which is gathered through cellular and other communication networks, has the disadvantage that agencies do not generally have direct access, 
but must procure through some type of negotiated data subscription. There are also privacy concerns related to traveler GPS information which have not been fully reconciled against potential benefits of the data.

- The connected vehicle initiative is a federal initiative that includes investment in development of a shared data format, dedication of spectrum for communication, and investment in resources to build test beds to develop the technology. As of 2017, the auto industry had launched the first vehicle model to have the on-board equipment to broadcast basic safety messages (BSMs) and receive signal phase and timing information (SPaT) from roadside equipment. In the public domain, the National Operations Center of Excellence has recently launched the "SPaT Challenge”, to encourage each state in the US to implement broadcasting of SPaT messages at approximately 20 intersections by the year 2020. The advantage of connected vehicle data is that agencies will have control of the data; the disadvantage is that a substantial infrastructure investment is needed and the same privacy concerns that have affected the use of broadly collected GPS information also apply. As a result of privacy concerns, the connective infrastructure has been designed such that individual vehicles will be traceable through an intersection, but they would not generally be traceable through a network.

A substantial amount of work remains to be done before the potential of these data sets can be fully known and whether they will supplant or augment existing methods. At the time of writing, the sample rates achievable by either of these emerging methods remains much lower than prevailing Bluetooth and Wi-Fi re-identification sensing rates and the overall accuracy of metrics calculated from broadly-collected GPS data is unknown. Connected vehicle data, if fully deployed will have excellent spatial and temporal resolution, but for privacy reasons, the analysis scope of the data will likely be initially limited to individual intersections. Currently, research emanating from laboratory simulations of this data shows promise, but it is unclear whether real-world data will match laboratory results. In addition, the rate at which the technology will proliferate remains to be seen.

In summary, even as new data collection technology continues to evolve and mature, the processes and methods introduced for arterial performance characterization using data from automated re-identification based sensing such as used with Bluetooth and WiFi are equally valid with new data streams. The quality of flow metrics applied within this project, whether from high-resolution controller data or from reidentification data, can be applied to other high-density data streams that provide sufficient vehicle position and trajectory data content. If these new methods are successful, they may provide the means to cost effectively scale arterial performance management nationwide.

\subsection{High-Resolution Data and Automated Traffic Signal Performance Measures}

High-resolution data has attracted an increasing amount of attention in the past few years. In 2013, Utah DOT began to invest resources into developing a system for automatically downloading the data and providing the performance measures using a web-based system. The name "Automated Traffic Signal Performance Measures" (ATSPMs) was adopted for the overall concept, and the software for the website has been published as open-source code on FHWA's Open Source Application Development Portal (OSADP) [18]. The efforts by Utah DOT were showcased by selection as a focus technology by the AASHTO Innovation Initiative [19]. 
Figure 5.1 shows a view of the Utah DOT website for Automated Traffic Signal Performance Measures (ATSPMs). The upper portions of the page show a menu for selecting an intersection from a map or a list, and for filtering the intersections shown by region or available metrics. The user also specifies a particular metric, some parameters controlling it, and a date for analysis. The bottom of the page is where the metrics are displayed. This example shows PCDs for two approaches at a particular intersection. At the time of writing, several agencies had implemented this software for their own systems, while a number of vendors had developed similar software or had adapted the open-source code into their own systems.
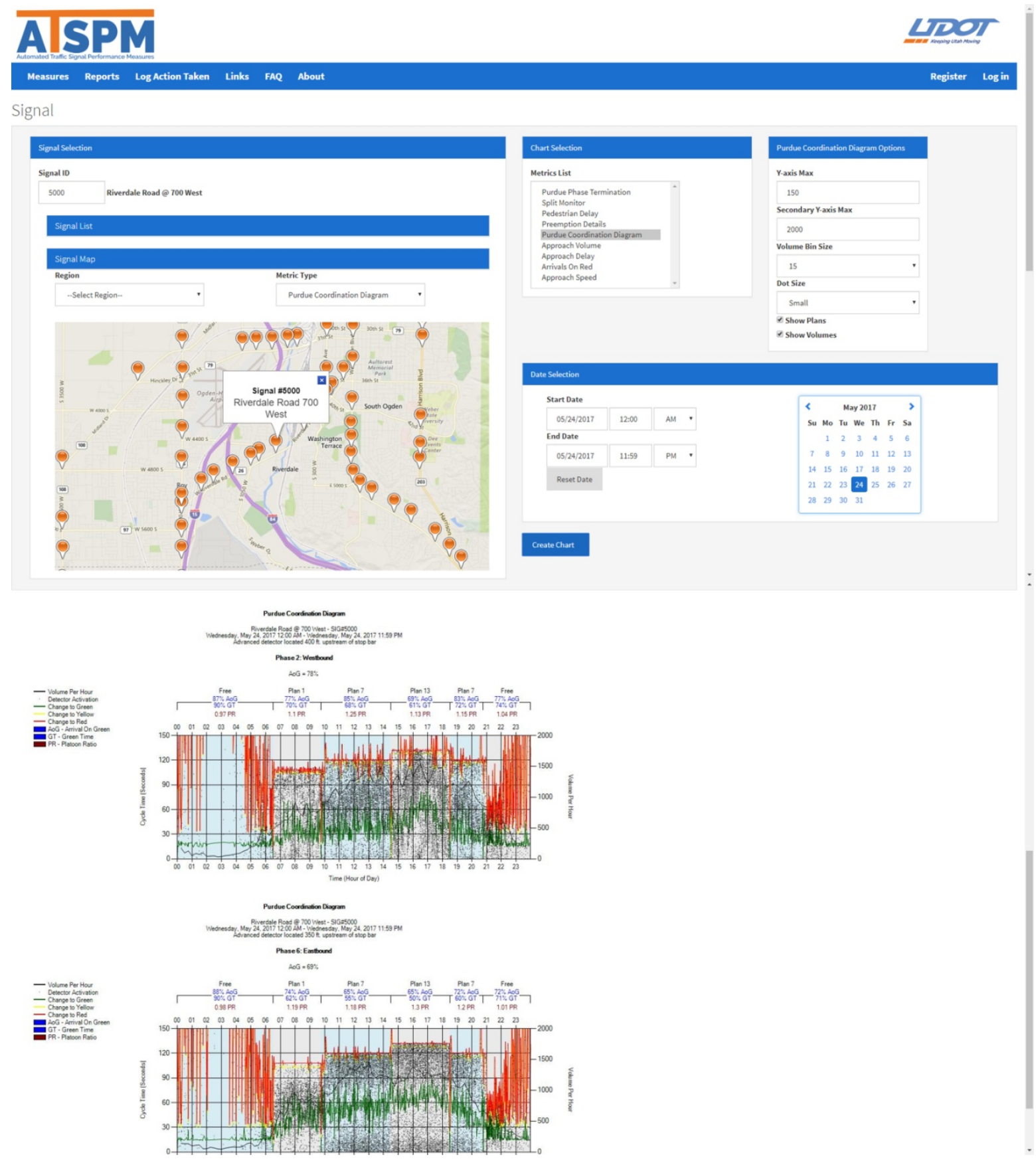

Figure 5.1 View of Utah DOT's Automated Traffic Signal Performance Measures website (http://udottraffic.utah.gov/atspm/) 
As of 2017, ATSPMs had been selected as an implementation initiative by FHWA for its Every Day Counts program [20], which means that federal funding resources are being used to help agencies implement their own systems. Further, at the time of writing, the National Academies has an active project, NCHRP 03-122, which will develop guidance for those agencies to use in such implementations. Thus, ATSPMs will likely continue to see additional usage by agencies. Although the data concepts have been around for over a decade, widespread usage of ATSPMs is a nascent development. Agency experience with ATSPMs will likely influence the future direction of the technology. As with reidentification technology, the potential for wide-spread adoption of either connected vehicle, or highdensity GPS data may provide more cost effective means to scale ATSPMs without the needs for costly investment and maintenance of associated field hardware. Also (similar to travel time distribution measures) the fundamental advancements enabled by use of high-resolution controllers will be applicable and valid independent of the source of the enabling field data.

\subsection{New Methods of Arterial System Management}

The research discussed in the present report is particularly timely in light of these developments. As more agencies begin adding data collection capabilities to their systems and begin developing data sets of their own similar to those described above, there will be a need to apply these in an arterial management program. To do so, there will be a need to integrate the data in such a way as to make decisions. The current tools, such as the ATSPM website discussed above, are able to provide the data for one particular location at a time. In other related tools, travel time information would typically be provided for a single corridor. The user must piece together the information to develop a view of the overall system. The methods of aggregation and the concepts of threshold and target setting of different facilities to develop an overall system score, as discussed in this research, would be applicable to the more granular data available in present systems.

With current developments in mind, we can return to the vision presented at the beginning of this report (repeated in Figure 5.2 below), which shows the development of arterial management systems relative to pavement management systems. The current trends, which have made considerable progress even since the beginning of this research project, reflect the development of standard data collection methodologies. These are starting to congeal into a few methods that seem likely to achieve widespread acceptance. The present research introduces concepts relevant to the development of the upper three activitiesdevelopment of management systems and integration into engineering practices. With the elements of the system coming into place, the dashed line in Figure 5.2 could be realized in the next few years, revolutionizing arterial systems management through addition of data-driven means of intelligence gathering and decision making. 


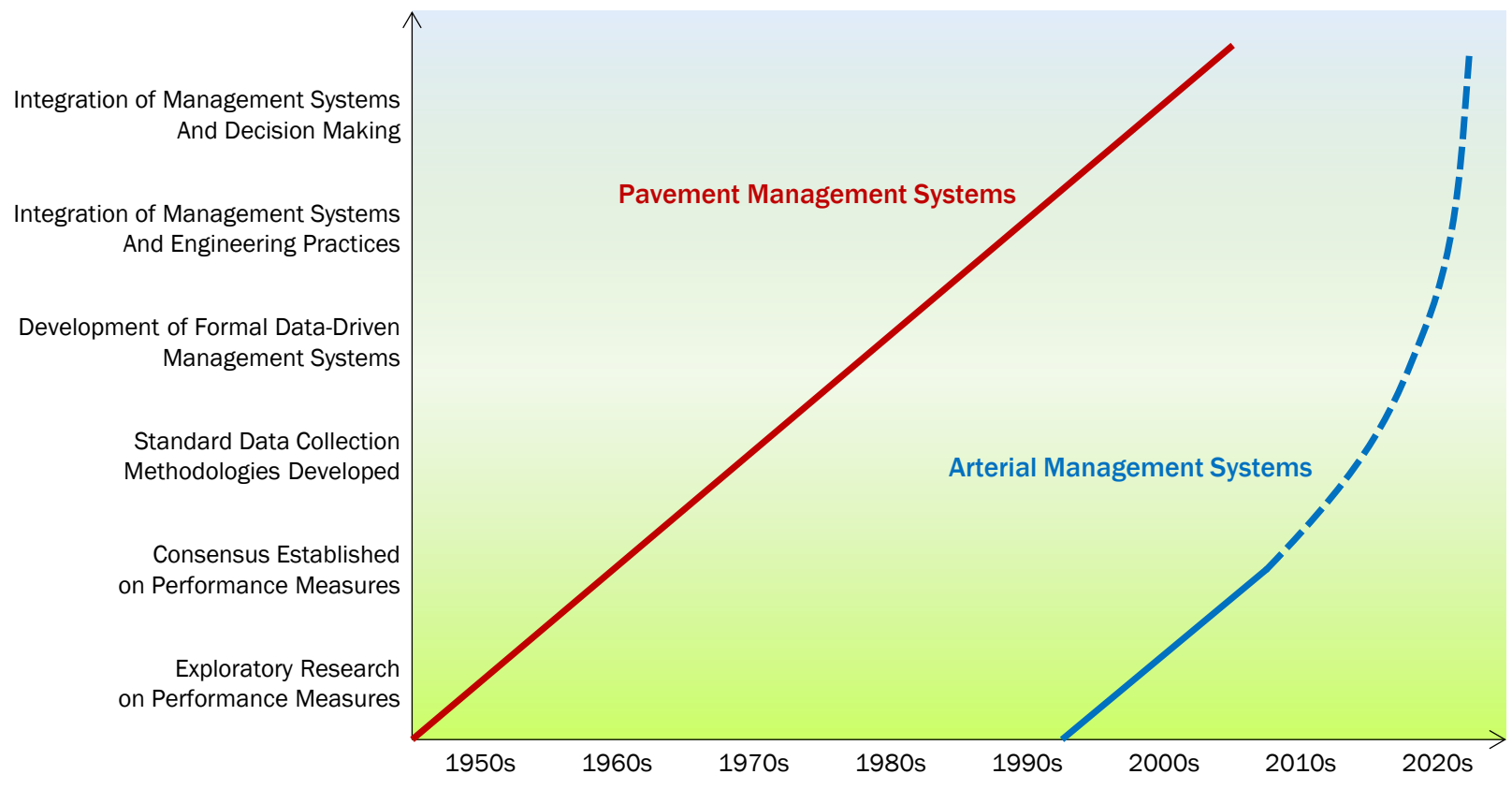

Figure 5.2 Evolution of pavement management systems and arterial management systems

To reach that goal a look toward future needs and research is needed in order to continue to quickly evolve signal management to data driven performance practices. The rapid adoption of high-resolution controller data and re-identification data, and more importantly the performance measures enabled by these technologies is beginning to fundamentally change the arterial signal management practice. As this trend continues, the maturation of the process will require additional tools and changes in business practice. Research using new data sources is already underway as previously described. This data rich world will require a number of new tools and practices to efficiently manage.

- Additional standards work such that data created from one vendor can be ported to many tools, ensuring longer data shelf life and more robust and cost efficient industry support.

- Better data management and hosting practices. Arterial data collection is quickly bridging the 'Big Data' threshold in which the shear size of the data sets requires specialized knowledge for efficient analytics and visualization processing. Appropriate business models for federated data sharing practices among and between public jurisdiction and private industry is required to scale these practices nationwide.

- Business model innovation that allows jurisdictions, particularly smaller jurisdictions, to leverage these investments without the need for highly specialized staffing. Business models and best practices for outsourcing not only of equipment, but also management practices and the data management inherent in these new data-driven approaches.

The good news is that arterial signal management is not the only field to be rapidly evolving to data driven processes. The internet of things and other technology revolutions are causing business disruptions in parallel industries that can provide case studies and lessons learned to inform the traffic industry. From these lessons learned, the arterial management profession can learn how to overcome the challenges and leverage the opportunities presented in the ever evolving traffic data landscape moving forward. 


\section{Bibliography}

[1] "2012 National Traffic Signal Report Card: Technical Report," National Transportation Operations Coalition, 2012.

[2] Office of Highway Policy Information, "Highway Statistics 2000," Federal Highway Administration, 2001.

[3] Highway Capacity Manual, National Academy of Sciences, 2000.

[4] C. Day, J. Sturdevant and D. Bullock, "Outcome Oriented Performance Measures for Management of Signalized Arterial Capacity," Transportation Research Record No. 2192, pp. 24-36, 2010.

[5] R. Freije, A. Hainen, A. Stevens, H. Li, W. Smith, C. Day, J. Sturdevant and D. Bullock, "Graphical Performance Measures for Practitioners to Triage Split Failure Trouble Calls," Transportation Research Record No. 2439, pp. 27-40, 2014.

[6] C. Day, R. Haseman, H. Premachandra, T. Brennan, J. Wasson, J. Sturdevant and D. Bullock, "Evaluation of Arterial Signal Coordination: Methodologies for Visualizing High-Resolution Event Data and Measuring Travel Time," Transportation Research Record No. 2192, pp. 37-49, 2010.

[7] C. Day, T. Brennan, A. Hainen, S. Remias, H. Premachandra, J. Sturdevant, G. Richards, J. Wasson and D. Bullock, "Reliability, Flexibility, and Environmental Impact of Alternative Objective Functions for Arterial Offset Optimization," Transportation Research Record No. 2259, pp. 8-22, 2011.

[8] H. Liu and W. Ma, "A Virtual Vehicle Probe Moel for Time-Dependent Travel Time Estimation on Signalized Arterials," Transportation Research Part C, vol. 17, pp. 11-26, 2009.

[9] S. Lavrenz, C. Day, J. Grossman, R. Freije and D. Bullock, "Use of High-Resolution Signal Controller Data to Identify Red Light Running," Transportation Research Record No. 2258, pp. 4153, 2016.

[10] H. Li, S. Lavrenz, C. Day, A. Stevens, J. Sturdevant and D. Bullock, "Use of Both Travel Time and Travel Time Reliability Measures to Quantify Benefits of Signal Timing Maintenance and Optimization," Transportation Research Record No. 2487, pp. 31-43, 2015.

[11] S. Quayle, P. Koonce, D. DePencier and D. Bullock, "Arterial Performance Measures with Media Access Control Readers: Portland, Oregon Pilot Study," Transportation Research Record No. 2192, pp. 185-193, 2010.

[12] S. Remias, A. Hainen, C. Day, T. Brennan, H. Li, E. Rivera-Hernandez, J. Sturdevant, S. Young and D. Bullock, "Performance Characterization of Arterial Traffic Flow Using Probe Vehicle Data," 
Transportation Research Record No. 2380, pp. 10-21, 2013.

[13] A. Hainen, J. Wasson, S. Hubbard, S. Remias, G. Farnsworth and D. Bullock, "Estimating Route Choice and Travel Time Reliability with Field Observations of Bluetooth Probe Vehicles," Transportation Research Record No. 2256, pp. 43-50, 2011.

[14] J. Sturdevant, T. Overman, E. Raamot, R. Deer, D. Miller, D. Bullock, C. Day, B. T., H. Li, A. Hainen and S. Remias, "Indiana Traffic Signal Hi Resolution Data Logger Enumerations," Indiana Department of Transportation and Purdue University, 2012.

[15] S. E. Young and D. So Ting Fong, "Arterial Performance Measures Software," SBIR Phase 3 Federal Highway Administration Project "Sensor Fusion and MOE Development for Off-Line Traffic Analysis of Real Time Data, February 13, 2017.

[16] S. E. Young and D. So Ting Fong, "Network Performance Measures for Arterials - a Systematic Level Perspective," SBIR Phase 3 Federal Highway Administration Project "Sensor Fusion and MOE Development for Off-Line Traffic Analysis of Real Time Data, June 3, 2016.

[17] S. E. Young and D. So Ting Fong, "Arterial Trip Length Characteristics Software," 2017.

[18] "Automated Traffic Signal Performance Measures (ATSPM)," [Online]. Available: https://www.itsforge.net/index.php/community/explore-applications\#/30/129. [Accessed 28 May 2017].

[19] AASHTO, "Aashto Innovation Initiative: Automated Traffic Signal Performance Measures," [Online].

Available: http://aii.transportation.org/Pages/AutomatedTrafficSignalPerformanceMeasures.aspx. [Accessed 28 May 2017].

[20] FHWA, "Automated Traffic Signal Performance Measures (ATSPMs)," [Online]. Available: https://www.fhwa.dot.gov/innovation/everydaycounts/edc_4/atspm.cfm. [Accessed 28 May 2017].

[21] C. M. Day and D. M. Bullock, "Performance Measures of Interrupted-Flow Roadways using ReIdentification and Signal Controller Data," FHWA SBIR 3, 2015.

[22] N. Abedi, A. Bhaskhar and E. Chung, "Bluetooth and Wi-Fi MAC Address Based Crowd Data Collection and Monitoring: Benefits, Challenges, and Enhancement," in Australian Transport Research Forum 2013 Proceedings, Brisbane, Australia, 2013. 


\section{Report Sponsor}

The "Small Business Innovation Development Act of 1982" (Pub. L. No. 97-219), along with reauthorizing legislation (Pub. L. No. 99-443 and Pub. L. No. 102-564, the "Small Business Research and Development Enhancement Act of 1992"), seeks to encourage the initiative of the private sector and to use small business effectively to meet federal research and development objectives. To comply with statutory obligations of the Act, the U.S. Department of Transportation established the Small Business Innovation Research (SBIR) Program, which conforms to the guidelines and regulations provided by the Small Business Administration. Annually, small businesses are solicited to submit innovative research proposals that address the high-priority requirements of the U.S. Department of Transportation and that have potential for commercialization.

This report was developed through a partnership between Traffax, Inc., and Purdue University with funding from a Phase III SBIR contract (DTFH6114C00035) with the Federal Highway Administration. The project, entitled "Sensor Fusion and MOE Development for Off-Line Traffic Analysis of Real Time Data," created and refined methods and tools for the characterization of performance along arterial corridors.

\section{Publication}

This report is part of a series of reports published in collaboration with USDOT, Traffax, Inc., and Purdue University. The full report series is available for download at http://docs.lib.purdue .edu/apmtp/.

\section{Open Access and Collaboration with Purdue University}

The Indiana legislature established the Joint Highway Research Project in 1937. In 1997, this collaborative venture between the Indiana Department of Transportation and Purdue University was renamed as the Joint Transportation Research Program (JTRP) to reflect state and national efforts to integrate the management and operation of various transportation modes. Since 1937, the JTRP program has published over 1,600 technical reports. In 2010, the JTRP partnered with the Purdue University Libraries to incorporate these technical reports in the University's open access digital repository and to develop production processes for rapidly disseminating new research reports via this repository. Affiliated publications have also recently been added to the collection. As of 2017, the JTRP collection had over 1.5 million downloads, with some particularly popular reports having over 20,000 downloads. 